\title{
Spirocyclization reactions and antiproliferative activity of indole phytoalexins 1-methoxybrassinin and its 1-substituted derivatives
}

\author{
Mariana Budovská,*a Martina Bago Pilátová,b Viera Tischlerová, ${ }^{b}$ and Ján Mojžišs \\ ${ }^{a}$ Department of Organic Chemistry, Institute of Chemical Sciences, Faculty of Science, \\ P. J. Šafárik University, Moyzesova 11, 040 01, Košice, Slovak Republic \\ ${ }^{b}$ Department of Pharmacology, Faculty of Medicine, P. J. Šafárik University, SNP 1, \\ 04066 Košice, Slovak Republic \\ E-mail: mariana.budovska@upjs.sk
}

DOI: https://doi.org/10.24820/ark.5550190.p009.958

\begin{abstract}
The effect of the reaction temperature and the solvent on the diastereoselectivity of the spirocyclization of 1-methoxybrassinin leading to 1-methoxyspirobrassinol methyl ether was studied. 1-Acyl derivatives of 1-methoxyspirobrassinol and 1-methoxyspirobrassinol methyl ether were prepared by the bromine-mediated spirocyclization reactions of derivatives of brassinin bearing an acyl group on the indole nitrogen with water or methanol as nucleophilic agents. The cyclization of 1-acyl derivatives of brassinin afforded the trans-diastereoisomer as the major product, whereas using 1-methoxybrassinin afforded the cis- and trans-isomers in a ratio near to 1:1. Bromospirocyclization of brassinin and 1-methylbrassinin in the presence of methanol resulted in the formation of spirobrassinin and 1-methylspirobrassinin. The newly synthesized analogues of indole phytoalexins exhibited more significant antiproliferative activity against human leukemia cell lines than the natural phytoalexins.
\end{abstract}

Keywords: Indole phytoalexins, spirocyclization, diastereoselectivity, 1-methoxyspirobrassinol methyl ether, antiproliferative activity

\section{Introduction}

In 1940 Müller first proposed the phytoalexin concept. ${ }^{1}$ Phytoalexins play a significant role in the defence response of plants. These secondary metabolities, which are synthesized de novo in response to biotic or abiotic stress, are part of the plant chemical defense mechanism. ${ }^{2}$ Indole phytoalexins produced by crucifers were first reported in 1986 by Takasugi. ${ }^{3}$ To date, 44 indole phytoalexins have been isolated from economically and dietary important plants of the family Cruciferae (syn Brassicaceae), which are cultivated worldwide (e.g. cabbage, turnip, Chinese cabbage, Japanese radish, wasabi, broccoli, rapeseed and arabidopsis). ${ }^{4}$ The 44 cruciferous phytoalexins have been divided into six groups according to simple structural features. ${ }^{4} \mathrm{~A}$ unique group of these natural products are spiroindoline structures containing a spirocyclic ring in the C-3 position $\left[(S)-(-)\right.$-spirobrassinin $[(-)-1],{ }^{5}(R)-(+)-1-$ methoxyspirobrassinin $[(+)-2],{ }^{6} 1-$ 
methoxyspirobrassinol $(\mathbf{3})^{7}$ and trans-(2R,3R)-(-)-1-methoxyspirobrassinol methyl ether $\left[(2 R, 3 R)-(-)-\mathbf{4 a}\right.$, (Figure 1)]. ${ }^{7}$ In 1987, the first spiroindoline phytoalexin $(S)-(-)$-spirobrassinin [(-)-1] was isolated from Pseudomonas cichorii-inoculated Japanese radish (Raphanus sativus). ${ }^{5}$ Natural (-)-spirobrassinin [(-)-1] was assigned the absolute configuration $(S)$ on the base of X-ray crystallographic analysis and CD studies. ${ }^{8,9}( \pm)$-Spirobrassinin $[( \pm)-1]$ was synthesized by thionyl chloride- or methanesulfonyl chloride-mediated cyclization of $( \pm)$ dioxibrassinin. ${ }^{8,9}$ A stereoselective synthesis of $(S)$-(-)-spirobrassinin [(-)-1] was achieved by bromine-induced spirocyclization of (-)-1-(8-phenylmenthoxycarbonyl)brassinin with water, followed by oxidation and removal of the chiral auxiliary. ${ }^{10}(R)-(+)-1-$ Methoxyspirobrassinin $[(+)-2]$ was isolated in 1994 from kohlrabi after UV irradiation ${ }^{6}$ while trans-(2R,3R)-(-)-1methoxyspirobrassinol methyl ether $[(2 R, 3 R)-(-)-4 a]$ and optically inactive 1methoxyspirobrassinol (3) were isolated in 1995 from Japanese radish after inoculation with Pseudomonas cichorii. ${ }^{7}$ Compound $\mathbf{3}$ exists in solution as a mixture of two diastereoisomers trans-3a and cis-3b in a 1:4 ratio, owing to its unstable hemiaminal structure. ${ }^{7}$ The enantiomers of ( \pm -1-methoxyspirobrassinin $[( \pm)-2]$ and trans- $( \pm)-1$-methoxyspirobrassinol methyl ether [trans- $( \pm)-4 a]$ were resolved by chiral HPLC and the absolute configurations of natural $(R)-(+)-$ 2 and $(2 R, 3 R)-(-)-4 a$ were determined by electronic circular dichroism (ECD), vibrational circular dichroism (VCD) and chemical correlation. ${ }^{11}$

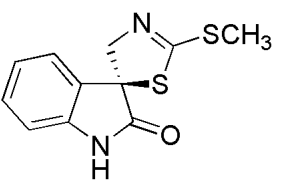

$(S)-(-) 1$

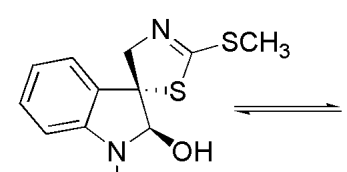

$\stackrel{\mathrm{O}}{\mathrm{O}} \mathrm{CH}_{3}$

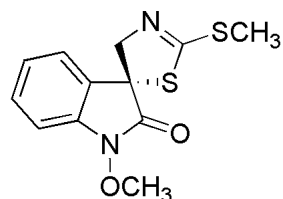

$(R)-(+)-(2)$

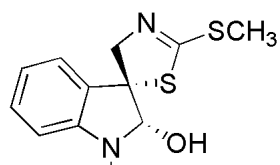

$( \pm)-3 \mathbf{b}$
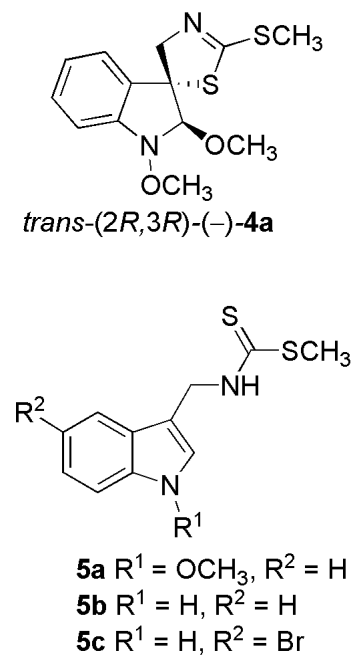

Figure 1. Selected indolic phytoalexins.

The first syntheses of 1-methoxyspirobrassinol (3) and 1-methoxyspirobrassinol methyl ether (4) were achieved by the 1,4-dioxane-dibromide (DDB)-mediated spirocyclization of 1methoxybrassinin (5a) in 1,4-dioxane in the presence of water or methanol. The reaction probably proceeds via sulfenyl bromide $\mathbf{6}$, which cyclizes to spiroindoleninium ion $\mathbf{A}$. When 1-methoxybrassinin (5a) was cyclized in the presence of water, 1-methoxyspirobrassinol [trans-( \pm$)-3 \mathbf{a}]$ and $[$ cis- $( \pm)-3 \mathbf{b}]$ was produced. In the presence of methanol as a nucleophile, a mixture of diastereoisomers, natural trans-( \pm -4a and unnatural cis-diastereoisomer cis- $( \pm)-\mathbf{4 b}$ in a ratio 1:2, was obtained (Scheme 1). ${ }^{12}$ Oxidation of a mixture of diastereoisomers trans- 
( \pm -3a and cis- $( \pm)-\mathbf{3 b}$ with $\mathrm{CrO}_{3}{ }^{12}$ or a mixture of diastereoisomers trans-( \pm )-4a and cis- $( \pm)-\mathbf{4 b}$ with PCC afforded racemic 1-methoxyspirobrassinin [( \pm$)-2] .{ }^{11}$

Indole phytoalexins have been previously shown to exert antibacterial, antifungal ${ }^{5-7,13,14}$ and anticancer properties ${ }^{11,15-18}$ and can serve as lead compounds for anticancer drug design. Brassinin (5b) and spirobrassinin [( \pm -1] $]$ are effective in inhibiting the formation of 7,12dimethylbenz $[a]$ anthracene (DMBA)-induced preneoplastic lesions in a mouse mammary gland. ${ }^{17}$ In addition, brassinin (5b) has been reported to exhibit dose-dependent inhibition of DMBA-induced and TPA-promoted skin carcinogenesis. ${ }^{19}$ Izutani et al. demostrated that brassinin (5b) inhibits cell growth in human colon cancer cells by arresting the $\mathrm{G}_{1}$ phase via inceased expression of p21 and p27. ${ }^{20}$ Spiroindoline phytoalexins and their derivatives exhibit an antiproliferative effect against human cancer cell lines. ${ }^{15,21-26}$ Brassinin (5b) and its synthetic derivative 5-bromobrassinin (5c) act as bioavailable competitive inhibitors of indoleamine 2,3dioxygenase (IDO), a tryptophan-catabolizing enzyme that promotes tumor escape via a mechanism of immune tolerance. ${ }^{27,28}$ 1-Methoxybrassinin (5a) displayed significant antiproliferative effect on intramolecular amastigotes of Trypanosoma cruzi and demonstrated a higher potency than shown by the currently used antichagasic agents (nifurtimox, benznidazol). ${ }^{29}$ Kristofikova et al. documented the in vitro effect of anti-aggregation of spirobrassinin $[( \pm)-1]$ in the cerebrospinal fluid of patients with multiple sclerosis. ${ }^{30}$

In this paper we describe our investigations into the diastereoselectivity of spirocyclization of 1-methoxybrassinin (5a) in the presence of various alcohols as nucleophiles, in various solvents and at various temperatures. We have also examined the bromine-initiated spirocyclization of 1-acyl derivatives of brassinin in the presence of water and methanol and studied the influence of acyl groups on the diastereoselectivity of bromospirocyclizations in comparison with 1-methoxybrassinin (5a). To our knowledge, no spiroindoline derivatives had been prepared by bromocyclization of brassinin $(\mathbf{5 b})$.

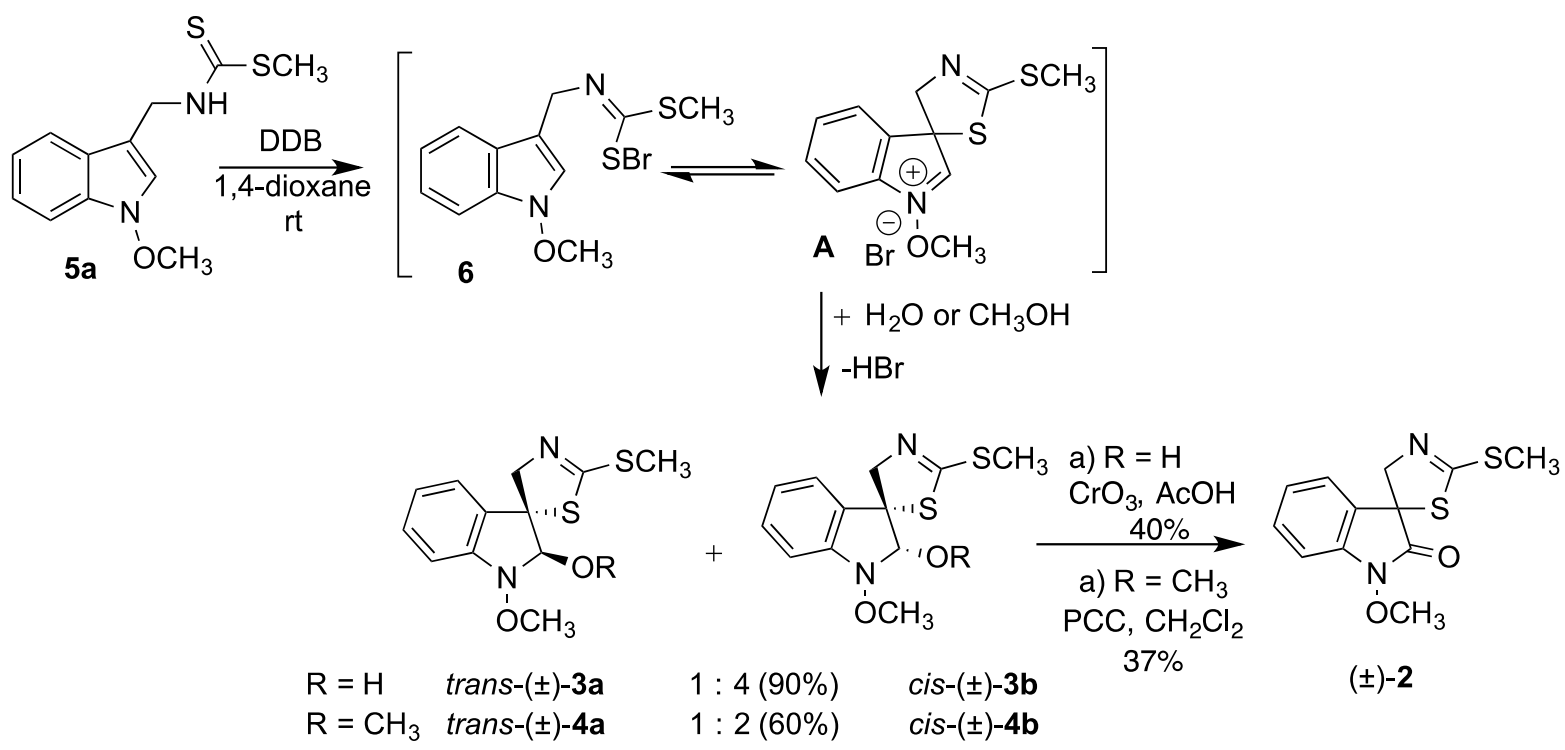

Scheme 1. Spirocyclization of 1-methoxybrassinin (5a) in the presence of water or methanol. 


\section{Results and Discussion}

First the effect of the solvent (dichloromethane, diethyl ether, diisopropyl ether, tetrahydrofuran, 1,4-dioxane) was studied on the diastereoselectivity of the bromine-induced spirocyclization reaction of 1-methoxybrassinin (5a) at rt. A distinct change in diastereoselectivity was observed upon replacing 1,4-dioxane as a solvent with dichloromethane. Bromine was used instead of 1,4-dioxane dibromide as a convenient cyclization agent. The reaction mixture was stirred for $15 \mathrm{~min}$ at $\mathrm{rt}$ and then triethylamine was added to trap the hydrogen bromide liberated during the reaction. Under these conditions a mixture of isomers containing a small excess of the trans-diastereoisomer trans-( \pm -4a was obtained (Table 1, entry 1). This result highlights the influence of the solvent on the diastereoselectivity of spirocyclization. When using 1,4-dioxane it is postulated that rather than direct addition of methanol on the methoxyiminium ion $\mathbf{A}$, which is favourable from both sides, a solvent molecule attacks methoxyiminium ion $\mathbf{A}$ from the less hindered thiazoline $\mathrm{CH}_{2}$ side with the formation of an unstable oxonium ion B. ${ }^{2}$ Subsequently methanol attacks the oxonium ion $\mathbf{B}^{2}$ from the sulfur side, which results in the formation of the cis-diastereoisomer cis- $( \pm)-$ 4b (Scheme 2).

The designations trans- and cis-diastereoisomers are used for differentiation of diastereoisomers. The trans-diastereoisomer is regarded as the one with the sulfur of thiazoline ring and methoxy group at C-2 located on the opposite sides of indoline ring, whereas the cisdiastereoisomer has the sulfur and 2-methoxy group on the same side of indoline ring.

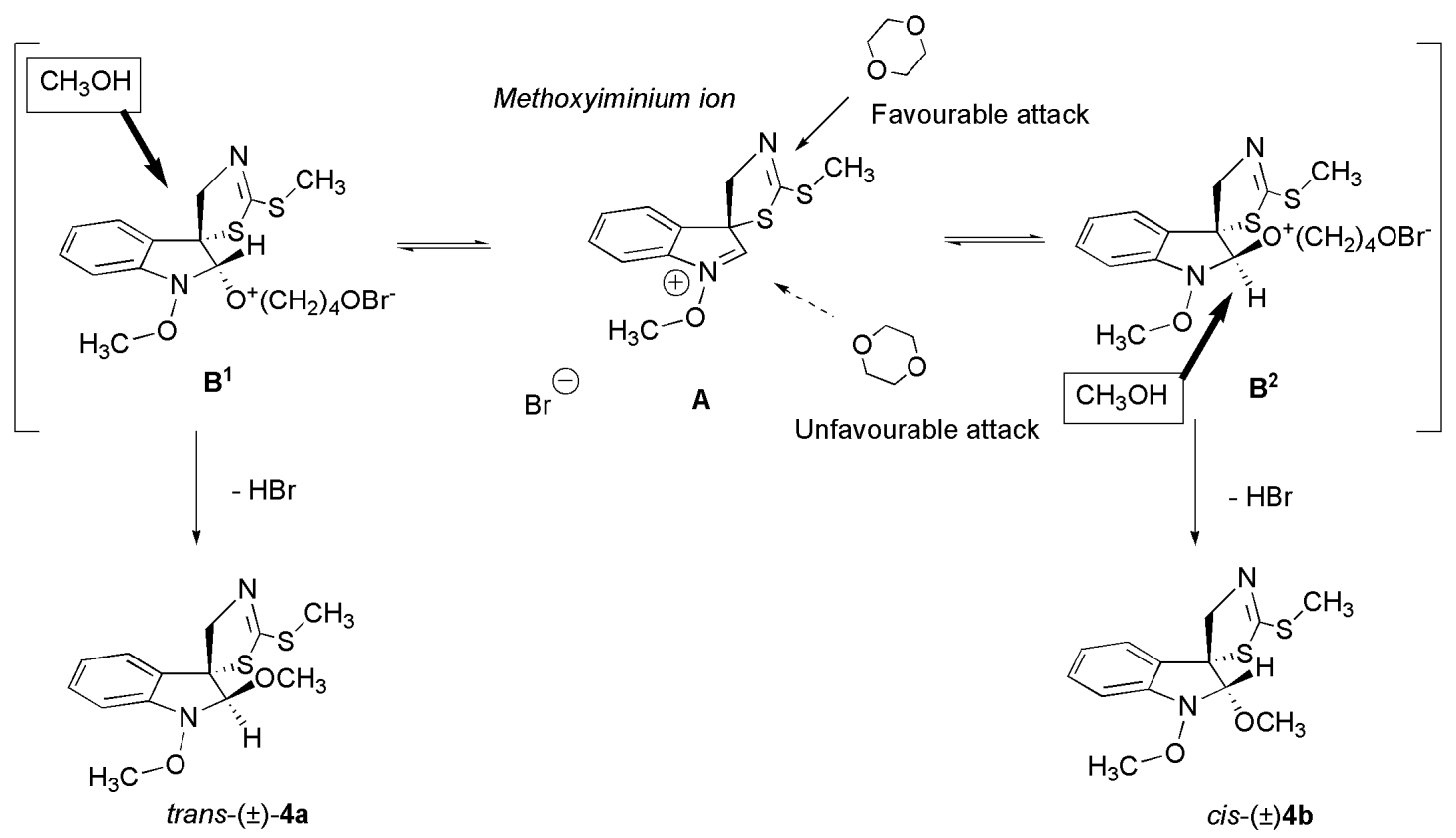

Scheme 2. The mechanism of the spirocyclization of 1-methoxybrassinin (5a) in 1,4-dioxane.

This mechanism was also supported by cyclizations with other ethers used as solvent (diethyl ether, diisopropyl ether and tetrahydrofuran). The trans-diastereoisomer trans-( \pm )-4a was obtained as the minor product (Table 1, entries 3 and 4) using diisopropyl ether and 
tetrahydrofuran, whereas the use of diethyl ether provided a 1:1 mixture of diastereoisomers trans- $( \pm)-\mathbf{4 a}$ and cis- $( \pm)-\mathbf{4 b}$ (Table 1, entry 2). Cyclization reactions carried out in methanol and $n$-heptane (Table 1, entries 6 and 7), in which methanol directly attacks the methoxyiminium ion A, provided a 1:1 mixture of trans $-( \pm)-\mathbf{4 a}$ and cis- $( \pm)-\mathbf{4 b}$ diastereoisomers as expected.

The effect of triethylamine on the diastereoselectivity of spirocyclization of 1methoxybrassinin (5a) was also determined. Performing the spirocyclization with bromine in anhydrous dichloromethane and subsequent addition of a solution of $\mathrm{Et}_{3} \mathrm{~N}$ in methanol, 1methoxyspirobrassinol methyl ethers were isolated in a 39:61 ratio in favor of cisdiastereoisomer cis-( \pm )-4b (Table 1, entry 8). It is postulated that in this case the $\mathrm{Et}_{3} \mathrm{~N}$ preferably approaches the intermediate methoxyiminium ion $\mathbf{A}$ from the less hindered $\mathrm{CH}_{2}$ side of thiazoline ring with the formation of an unstable triethylammonium ion analogous to that produced from 1,4-dioxane.

Table 1. The effect of solvent on the diastereoselectivity of the spirocyclization of the 1methoxybrassinin (5a) at room temperature

\begin{tabular}{|c|c|c|c|}
\hline Entry & Conditions & $\begin{array}{c}\text { Ratio }^{\mathrm{a}} \\
\operatorname{trans}^{-}( \pm)-\mathbf{4 a} \\
\text { cis }-( \pm)-\mathbf{4 b}\end{array}$ & $\begin{array}{l}\text { Yield }^{\mathrm{b}} \\
(\%)\end{array}$ \\
\hline 1 & $\mathrm{Br}_{2}, \mathrm{CH}_{2} \mathrm{Cl}_{2} / \mathrm{MeOH}$ (v:v 9:1) & $54: 46$ & 65 \\
\hline 2 & $\mathrm{Br}_{2}, \mathrm{Et}_{2} \mathrm{O} / \mathrm{MeOH}(\mathrm{v}: \mathrm{v} 9: 1)$ & $50: 50$ & 67 \\
\hline 3 & $\mathrm{Br}_{2}$, diisopropyl ether/MeOH (v:v 9:1) & $40: 60$ & 73 \\
\hline 4 & $\mathrm{Br}_{2}, \mathrm{THF} / \mathrm{MeOH}(\mathrm{v}: \mathrm{v} 9: 1)$ & $43: 57$ & 67 \\
\hline 5 & DDB, 1,4-dioxane/MeOH (v:v 9:1) & $36: 64^{12}$ & 60 \\
\hline 6 & $\mathrm{Br}_{2}, \mathrm{MeOH}(\mathrm{v}: \mathrm{v} 9: 1)$ & $50: 50$ & 76 \\
\hline 7 & $\mathrm{Br}_{2}, n$-heptane/MeOH (v:v 9:1) & $50: 50$ & 67 \\
\hline 8 & $\begin{array}{l}\mathrm{Br}_{2}, \mathrm{CH}_{2} \mathrm{Cl}_{2} \text {, after } 1 \text { min. } 1.1 \text { eq. } \mathrm{MeOH}, 10 \text { eq. } \\
\mathrm{Et}_{3} \mathrm{~N}\end{array}$ & $39: 61$ & 89 \\
\hline
\end{tabular}

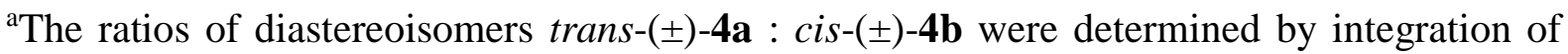
separate signals corresponding to $\mathrm{H}-2, \mathrm{H}_{\mathrm{a}}$ and $\mathrm{H}_{\mathrm{b}}$ protons in the ${ }^{1} \mathrm{H}$ NMR spectrum of the crude product mixture.

${ }^{\mathrm{b}}$ Crude product.

With the aim of influencing the diastereoselectivity of the spirocyclization of $\mathbf{5 a}$, the effect of temperature on the reaction was examined. Performing experiments above and below rt confirmed that temperature has a distinct effect on the diastereoselectivity of the spirocyclization of 1-methoxybrasssinin (5a). Reactions performed at low temperature led predominantly to the trans-diastereoisomer trans- $( \pm)-4 \mathbf{a}$ (Table 2$)$, whereas at $\mathrm{rt}$ or at $40-60{ }^{\circ} \mathrm{C}$ in 1,4-dioxane a preference for the cis-diastereoisomer cis- $( \pm)-\mathbf{4 b}$ was observed. The best ratio was achieved at $-70^{\circ} \mathrm{C}$ in THF as solvent (Table 2 , entry 16). 
Table 2. The effect of temperature on the diastereoselectivity of the spirocyclization of 1methoxybrassinin (5a)

\begin{tabular}{|c|c|c|c|c|}
\hline Entry & Conditions & Temperature & $\begin{array}{c}\text { Ratio }^{\mathrm{a}} \\
\text { trans }-( \pm)-\mathbf{4 a}: \text { cis }-( \pm)-\mathbf{4 b}\end{array}$ & $\begin{array}{c}\text { Yield }^{b} \\
(\%)\end{array}$ \\
\hline 1 & $\mathrm{Br}_{2}, \mathrm{CH}_{2} \mathrm{Cl}_{2} / \mathrm{MeOH}$ (v:v 9:1) & $\mathrm{rt}$ & $54: 46$ & 65 \\
\hline 2 & & $0{ }^{\circ} \mathrm{C}$ & $62: 38$ & 73 \\
\hline 3 & & $-20^{\circ} \mathrm{C}$ & $65: 35$ & 73 \\
\hline 4 & & $-60^{\circ} \mathrm{C}$ & $74: 26$ & 70 \\
\hline 5 & $\mathrm{Br}_{2}, \mathrm{Et}_{2} \mathrm{O} / \mathrm{MeOH}(\mathrm{v}: \mathrm{v} 9: 1)$ & $\mathrm{rt}$ & $50: 50$ & 67 \\
\hline 6 & & $0^{\circ} \mathrm{C}$ & $61: 39$ & 73 \\
\hline 7 & & $-20^{\circ} \mathrm{C}$ & $68: 32$ & 73 \\
\hline 8 & & $-60^{\circ} \mathrm{C}$ & $75: 25$ & 70 \\
\hline 9 & $\mathrm{Br}_{2}$, diisopropyl ether/MeOH & $\mathrm{rt}$ & $40: 60$ & 73 \\
\hline 10 & $(\mathrm{v}: \mathrm{v} 9: 1)$ & $0^{\circ} \mathrm{C}$ & $61: 39$ & 70 \\
\hline 11 & & $-20^{\circ} \mathrm{C}$ & $69: 31$ & 73 \\
\hline 12 & & $-60^{\circ} \mathrm{C}$ & $72: 28$ & 73 \\
\hline 13 & $\mathrm{Br}_{2}, \mathrm{THF} / \mathrm{MeOH}(\mathrm{v}: \mathrm{v} 9: 1)$ & $\mathrm{rt}$ & $43: 57$ & 67 \\
\hline 14 & & $0{ }^{\circ} \mathrm{C}$ & $68: 32$ & 70 \\
\hline 15 & & $-20^{\circ} \mathrm{C}$ & $70: 30$ & 70 \\
\hline 16 & & $-70^{\circ} \mathrm{C}$ & $85: 15$ & 67 \\
\hline 17 & DDB, 1,4-dioxane/MeOH (v:v & $60^{\circ} \mathrm{C}$ & $27: 73$ & 70 \\
\hline 18 & $9: 1)$ & $40^{\circ} \mathrm{C}$ & $28: 72$ & 70 \\
\hline 19 & & $\mathrm{rt}$ & $36: 64^{12}$ & 60 \\
\hline 20 & & $0{ }^{\circ} \mathrm{C}$ & $63: 37$ & 73 \\
\hline 21 & & $-20^{\circ} \mathrm{C}$ & $75: 25$ & 73 \\
\hline 22 & $\mathrm{Br}_{2}, \mathrm{MeOH}$ & $\mathrm{rt}$ & $50: 50$ & 76 \\
\hline 23 & & $-20^{\circ} \mathrm{C}$ & $61: 39$ & 73 \\
\hline 24 & & $-60^{\circ} \mathrm{C}$ & $70: 30$ & 73 \\
\hline 25 & $\mathrm{Br}_{2}, n$-heptane/MeOH (v:v 9:1) & $\mathrm{rt}$ & $50: 50$ & 67 \\
\hline 26 & & $-60^{\circ} \mathrm{C}$ & $63: 37$ & 71 \\
\hline 27 & $\begin{array}{l}\mathrm{Br}_{2}, n \text {-heptane, after } 1 \text { min. } 1.1 \\
\text { eq. } \mathrm{MeOH}\end{array}$ & $-60^{\circ} \mathrm{C}$ & $70: 30$ & 63 \\
\hline 28 & $\mathrm{Br}_{2}, \mathrm{CH}_{2} \mathrm{Cl}_{2}$, after $1 \mathrm{~min}$ & $\mathrm{rt}$ & $39: 61$ & 89 \\
\hline 29 & 1.1 eq. $\mathrm{MeOH}, 10$ eq. $\mathrm{Et}_{3} \mathrm{~N}$ & $-75^{\circ} \mathrm{C}$ & $17: 83$ & 88 \\
\hline
\end{tabular}

${ }^{\text {a }}$ The ratios of diastereoisomers trans- $( \pm)-\mathbf{4 a}$ : cis- $( \pm)-\mathbf{4 b}$ were determined by integration of separate signals corresponding to $\mathrm{H}-2, \mathrm{H}_{\mathrm{a}}$ and $\mathrm{H}_{\mathrm{b}}$ protons in the ${ }^{1} \mathrm{H}$ NMR spectrum of the crude product mixture.

${ }^{\mathrm{b}}$ Crude product.

Under these conditions a mixture of trans-diastereoisomer trans- $( \pm)-\mathbf{4 a}$ and cisdiastereoisomer cis- $( \pm)-\mathbf{4 b}$ was obtained in an 85:15 ratio. It is postulated that at low temperature, molecules of methanol form intermolecular hydrogen bonds with the solvent used 
as well as with each other to create bulky associates. Such associates reacts with the methoxyiminium ion $\mathbf{A}$ from the less hindered thiazoline $\mathrm{CH}_{2}$ side with the preferential formation of trans-diastereoisomer trans- $( \pm)-\mathbf{4 a}$.

Finding the effect of solvent on the diastereoselectivity of the spirocyclization of 5a led us to use methanol as a nucleophile in the form of a large complex. Therefore we performed the bromospirocyclization of 1-methoxybrassinin (5a) in anhydrous dichloromethane with sodium methoxide in the presence of 15-crown-5 ether. To a stirred mixture of 1-methoxybrassinin (5a) in anhydrous dichloromethane was added bromine. After stirring for one minute, a freshly prepared solution of the complex $\mathrm{CH}_{3} \mathrm{ONa} / 15$-crown-5 in anhydrous dichloromethane was added. In the product misture, cis-diastereoisomer cis- $( \pm)-\mathbf{4 b}$ predominated (Table 3 , entry 1$)$. Probably, the complex $\mathrm{CH}_{3} \mathrm{ONa} / 15$-crown-5-ether was decomposed by the influence of hydrogen bromide liberated during reaction and 15-crown-5-ether liberated from the complex had the same effect on the diastereoselectivity as did 1,4-dioxane. To prevent decomposition of this complex, triethylamine ( 2 eq.) was added to the reaction mixture to trap the hydrogen bromide and then a solution of complex $\mathrm{CH}_{3} \mathrm{ONa}$ /15-crown-5-ether in anhydrous dichloromethane was added. As can be seen from Table 3 (entry 2), this spirocyclization of $\mathbf{5 a}$ led to formation of the trans-diastereoisomer trans-( \pm -4a preferentially. Performing the spirocyclization with anhydrous $\mathrm{K}_{2} \mathrm{CO}_{3}$ (2 eq., Table 3, entry 3), the natural diastereoisomer of 1-methoxyspirobrassinol methyl ether trans- $( \pm)-\mathbf{4 a}$ was prepared with improved diastereoselectivity in a 69:31 ratio. Probably, the complex of sodium methoxide with 15crown-5-ether as nucleophile approaches the intermediate methoxyiminium ion $\mathbf{A}$ preferemtially from the less hindered $\mathrm{CH}_{2}$ side of thiazoline ring and leads to formation transdiastereoisomer trans- $( \pm)-\mathbf{4 a}$.

Table 3. Spirocyclization of the 1-methoxybrassinin (5a) in the presence of the complex $\mathrm{MeONa} / 15$-crown-5 ether at room temperature

\begin{tabular}{llcc}
\hline Entry & Conditions & $\begin{array}{c}\text { Ratio }^{\mathrm{a}} \\
\text { trans- }^{( \pm)}-\mathbf{4 a}: \text { cis- }( \pm)-\mathbf{4 b}\end{array}$ & $\begin{array}{c}\text { Yield }^{\mathrm{b}} \\
(\boldsymbol{\%})\end{array}$ \\
\hline 1 & $\mathrm{Br}_{2}, \mathrm{CH}_{2} \mathrm{Cl}_{2}, \mathrm{MeONa} / 15$-crown-5 & $39: 61$ & 68 \\
2 & $\mathrm{Br}_{2}, \mathrm{CH}_{2} \mathrm{Cl}_{2}, \mathrm{Et}_{3} \mathrm{~N}, \mathrm{MeONa} / 15-$ crown-5 & $64: 36$ & 71 \\
3 & $\mathrm{Br}_{2}, \mathrm{CH}_{2} \mathrm{Cl}_{2}, \mathrm{~K}_{2} \mathrm{CO}_{3}, \mathrm{MeONa} / 15$-crown-5 & $69: 31$ & $71^{11}$ \\
\hline
\end{tabular}

${ }^{\mathrm{a}}$ The ratios of diastereoisomers trans- $( \pm)-\mathbf{4 a}:$ cis $-( \pm)-\mathbf{4 b}$ were determined by integration of separate signals corresponding to $\mathrm{H}-2, \mathrm{H}_{\mathrm{a}}$ and $\mathrm{H}_{\mathrm{b}}$ protons in the ${ }^{1} \mathrm{H} \mathrm{NMR}$ spectrum of the crude product mixture. ${ }^{\mathrm{b}} \mathrm{Crude}$ product.

We also investigated the effect of the bulkiness of alcohols (ethanol, isopropyl alcohol, tertbutanol, phenol and naphth-2-ol) on the diastereoselectivity of the spirocyclization of 1methoxybrassinin (5a, Scheme 3). Diastereoisomers trans-( \pm )-7a, cis- $( \pm)-\mathbf{7 b}$ and trans $-( \pm)-\mathbf{9 a}$, cis-( \pm )-9b were obtained in a 57:43 ratio (Table 4, entries 2 and 4). The use of isopropyl alcohol provided a 62:38 mixture of isomers trans-( \pm -8a, cis- $( \pm)-8 \mathbf{b}$ (Table 4, entry 3$)$. The bromocyclization reaction of 1-methoxybrassinin (5a) in the presence of phenol and naphth-2ol as nucleophiles afforded mixtures of diastereoisomers $( \pm)-\mathbf{1 0 a}-( \pm)-\mathbf{1 0 b}$ and $( \pm)-\mathbf{1 1 a}-( \pm)-\mathbf{1 1} \mathbf{b}$ with a slight excess of the cis-isomer (Table 4, entries 5 and 6). 


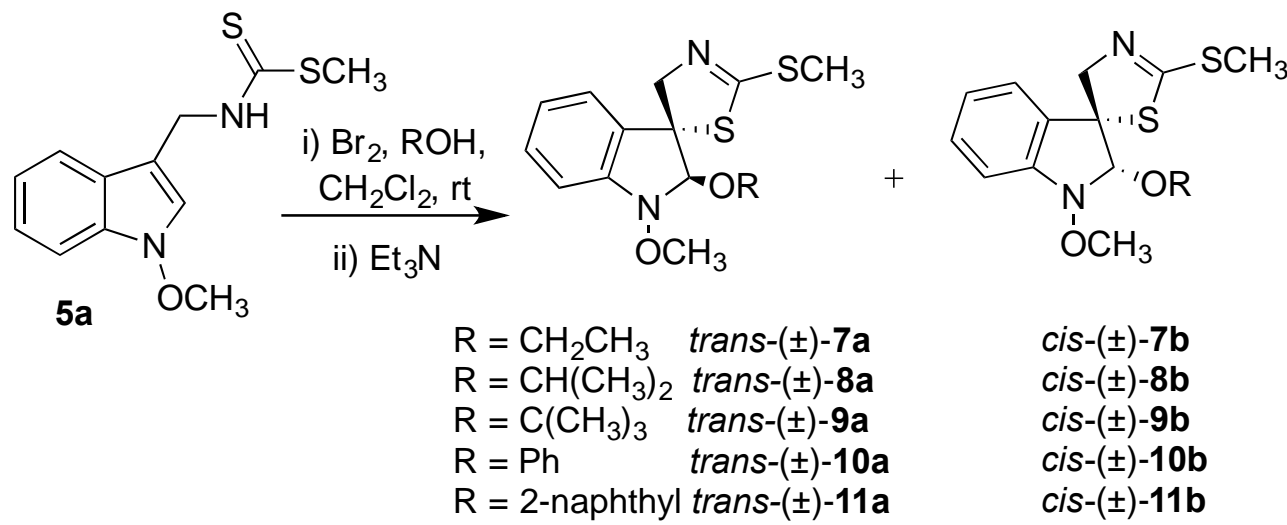

Scheme 3. Cyclization reactions of 1-methoxybrassinin (5a) with alcohols.

Table 4. The effect of the bulkiness of alcohols (ethanol, isopropyl alcohol, tert-butanol, phenol and naphth-2-ol) on the diastereoselectivity of spirocyclization of 1-methoxybrassinin (5a) at room temperature

\begin{tabular}{lllcc}
\hline Entry & Compound & $\mathrm{R}$ & $\begin{array}{c}\text { Ratio }^{\mathrm{a}} \\
\text { trans- }( \pm)-: \text { cis- }^{( \pm)-}\end{array}$ & $\begin{array}{c}\text { Yield }^{\mathrm{b}} \\
(\%)\end{array}$ \\
\hline 1 & $( \pm)-\mathbf{4 a},( \pm)-\mathbf{4 b}$ & $\mathrm{Me}$ & $54: 46$ & 65 \\
2 & $( \pm)-\mathbf{7 a},( \pm)-\mathbf{7 b}$ & $\mathrm{Et}$ & $57: 43$ & 68 \\
3 & $( \pm)-\mathbf{8 a},( \pm)-\mathbf{8 b}$ & $i$-Pr & $62: 38$ & 77 \\
4 & $( \pm)-\mathbf{9 a},( \pm)-\mathbf{9 b}$ & $t$-Bu & $57: 43$ & 71 \\
5 & $( \pm)-\mathbf{1 0 a},( \pm)-\mathbf{1 0 b}$ & $\mathrm{Ph}$ & $32: 68$ & $88^{23}$ \\
6 & $( \pm)-\mathbf{1 1 a},( \pm)-\mathbf{- 1 1 b}$ & 2-naphthyl & $32: 68$ & 65 \\
\hline
\end{tabular}

${ }^{\mathrm{a}}$ The ratios of diastereoisomers $( \pm)-\mathbf{7 a}:( \pm)-7 \mathbf{b}-( \pm)-11 \mathbf{a}:( \pm)-11 \mathbf{b}$ were determined by integration of separate signals corresponding to $\mathrm{H}-2, \mathrm{H}_{\mathrm{a}}$ and $\mathrm{H}_{\mathrm{b}}$ protons in the ${ }^{1} \mathrm{H}$ NMR spectrum of the crude product mixture. ${ }^{\mathrm{b}} \mathrm{Crude}$ product.

We also decided to study the influence of an acyl group on the diastereoselectivity of the bromospirocyclization reactions of 1-acylderivatives, 12, and 23-25, of brassinin. For the experiments we selected tert-butoxycarbonyl, acetyl, benzoyl and methoxycarbonyl groups. The key intermediate 1-Boc-brassinin (12) was prepared by the previously reported procedure. ${ }^{31}$ Commercially available indole-3-carboxaldehyde (13) was used as a starting compound for the preparation 1-acetyl (23), 1-benzoyl (24) and 1-(methoxycarbonyl)brassinin (25, Scheme 4). N-Acyl aldehydes 14-16 were synthesized by various methods, using acetic anhydride/DMAP in THF $(\mathbf{1 4}, 91 \%)$, benzoyl chloride/Et ${ }_{3} \mathrm{~N}$ in THF $(\mathbf{1 5}, 98 \%)$ or methyl chloroformate/ $\mathrm{NaH}$ in acetonitrile $(\mathbf{1 6}, 85 \%)$. Treatment of aldehydes 14 and 15 with hydroxylamine hydrochloride in THF in the presence of sodium acetate as the base provided oximes 17 and 18 as mixtures of $Z$ - and $E$-isomers. Oxime 19 was obtained from aldehyde 16 using $\mathrm{NH}_{2} \mathrm{OH} . \mathrm{HCl}, \mathrm{Na}_{2} \mathrm{CO}_{3}, \mathrm{EtOH}, \mathrm{H}_{2} \mathrm{O}$ in $80 \%$ yield. Nickel boride-catalyzed reduction of 17 using sodium borohydride afforded the unstable amine $\mathbf{2 0}$ which was employed as a crude product immediately after isolation. Subsequent reaction of amine 20 with $\mathrm{CS}_{2}$ and $\mathrm{CH}_{3} \mathrm{I}$ in 
methanol in the presence of $\mathrm{Et}_{3} \mathrm{~N}$ resulted in the formation of 1-acetylbrassinin (23) in $22 \%$ yield. A better result was obtained, when dichloromethane was used for the extraction of amine 20 and also as a solvent in the reaction with $\mathrm{CS}_{2}$ and $\mathrm{CH}_{3} \mathrm{I}$. Under these conditions 1acetylbrassinin (23) was isolated in $78 \%$ yield. The reduction of oxime 18 by sodium borohydride and subsequent treatment of amine 21 with $\mathrm{CS}_{2}$ and $\mathrm{CH}_{3} \mathrm{I}$ in methanol afforded 1benzoylbrassinin (24) in $20 \%$ yield. Replacement of the methanol as solvent by dichloromethane again improved the yield to $36 \%$ (Scheme 4). If we performed the reduction of 18 with sodium cyanoborohydride, the yield of 1-benzoylbrassinin (24) was 32\%.

1-(Methoxycarbonyl)brassinin (25) was prepared from the unstable 1-(methoxycarbonyl)indole-3-ylmethyl amine (22, obtained by nickel boride-catalyzed reduction of 19 with sodium borohydride) by the reaction with $\mathrm{CS}_{2}$ and $\mathrm{CH}_{3} \mathrm{I}$ in methanol in $58 \%$ yield after two reaction steps .

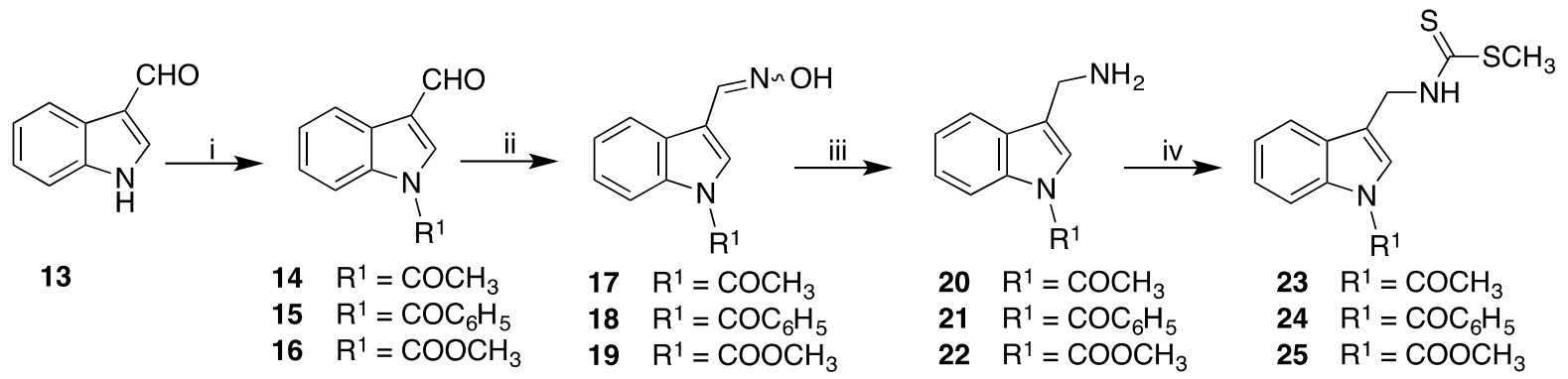

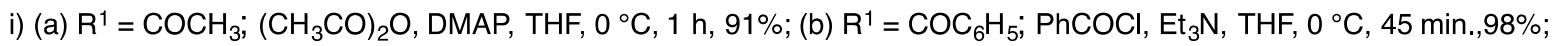

(c) $\mathrm{R}^{1}=\mathrm{COOCH}_{3} ; \mathrm{CH}_{3} \mathrm{OCOCl}, \mathrm{NaH}, \mathrm{CH}_{3} \mathrm{CN}, \mathrm{rt}, 10 \mathrm{~min}, 85 \%$;

ii) (a) $\mathrm{R}^{1}=\mathrm{COCH}_{3} ; \mathrm{NH}_{2} \mathrm{OH} . \mathrm{HCl}, \mathrm{CH}_{3} \mathrm{COONa}, \mathrm{THF} / \mathrm{H}_{2} \mathrm{O}$, rt, $4 \mathrm{~h}, 88 \%$; (b) $\mathrm{R}^{1}=\mathrm{COC}_{6} \mathrm{H}_{5} ; \mathrm{NH}_{2} \mathrm{OH} . \mathrm{HCl}, \mathrm{CH}_{3} \mathrm{COONa}$, $\mathrm{THF} / \mathrm{H}_{2} \mathrm{O}$, rt, $4 \mathrm{~h}, 88 \%$; (c) $\mathrm{R}^{1}=\mathrm{COOCH}_{3} ; \mathrm{NH}_{2} \mathrm{OH} . \mathrm{HCl}, \mathrm{Na}_{2} \mathrm{CO}_{3}, \mathrm{EtOH} / \mathrm{H}_{2} \mathrm{O}$, rt, 10 min, 80\%;

iii) $\mathrm{NiCl}_{2} \cdot 6 \mathrm{H}_{2} \mathrm{O}, \mathrm{NaBH}_{4}, \mathrm{MeOH}$;

iv) (a) $\mathrm{R}^{1}=\mathrm{COCH}_{3} ; \mathrm{Et}_{3} \mathrm{~N}, \mathrm{CS}_{2}, \mathrm{Mel}, \mathrm{CH}_{2} \mathrm{Cl}_{2}, \mathrm{rt}, 1 \mathrm{~h}, 78 \%$; (b) $\mathrm{R}^{1}=\mathrm{COC}_{6} \mathrm{H}_{5} ; \mathrm{Et}_{3} \mathrm{~N}, \mathrm{CS}_{2}, \mathrm{Mel} \mathrm{CH}_{2} \mathrm{Cl}_{2}, \mathrm{rt}, 30 \mathrm{~min}, 36 \%$; (c) $\mathrm{R}^{1}=\mathrm{COOCH}_{3} ; \mathrm{Et}_{3} \mathrm{~N}, \mathrm{CS}_{2}, \mathrm{Mel}, \mathrm{MeOH}, \mathrm{rt}, 15 \mathrm{~min}, 58 \%$.

Scheme 4. Synthesis of 1-acyl derivatives 12, 23-25 of brassinin.

Derivatives of brassinin 12, 23-25 were dissolved in a mixture dichloromethane/water or methanol (v/v 9:1) and then 1.1 equivalents of bromine were added (Scheme 5). After 15 minutes of stirring triethylamine was added for the neutralization of hydrogen bromide liberated by the spirocyclization. In such a way were prepared 1-acetyl- (26), 1-benzoyl- (27), 1-methoxycarbonyl- (28) and 1-Boc-spirobrassinol (29) as well as 1-acetyl- (30), 1-benzoyl(31), 1-methoxycarbonyl- (32) and 1-Boc-spirobrassinol methyl ether (33). The ratios of diastereoisomers $( \pm)-\mathbf{2 6 a}-( \pm)-\mathbf{3 3 b}$ and yields are summarized in Table 5 . In all cases the transdiastereoisomer trans- $( \pm)-26 a-( \pm)-33 a$ was obtained in preference. trans-Diastereoisomer trans-( \pm -33a was also the major product using 1,4-dioxane as the solvent. Cooling the reaction mixture did not change the stereoselectivity. The cyclization reaction of $\mathbf{1 2}$ accomplished at $60{ }^{\circ} \mathrm{C}$ also led predominantly to the trans-diastereoisomer trans-( \pm )-33a (Table 5). For comparison, Table 5 includes the ratios of diastereoisomers of 1-methoxyspirobrassinol $[( \pm)$ 3a- $( \pm)-3 \mathbf{b}]$ and 1-methoxyspirobrassinol methyl ether $[( \pm)-\mathbf{4 a}-( \pm)-\mathbf{4 b}]$. 
diastereoselectivity. Under the same conditions, trans-diastereoisomers predominated from 1acyl derivatives, whereas with 1-methoxybrassinin (5a) the cis- and trans-isomers were obtained in a near 1: 1 ratio (Tables 1, 4 and 5). The diastereoselectivity can be explained by a different mechanism. In both cases reactions probably start at the thiocarbamoyl group creating a sulfenyl bromide $\mathbf{6}, \mathbf{3 5}$. In the case of the methoxy derivative, the sulfenyl bromide $\mathbf{6}$ undergoes electrophilic attack on the sulfur with the formation of 1-methoxyspiroindoleninium intermediate A. Subsequent nucleophilic addition of methanol gives spiroindoline structures trans-( \pm )-4a, cis- $( \pm)-\mathbf{4 b}$ (Scheme 6$)$. In the case of the 1-acyl derivatives, delocalization of the lone electron pair on nitrogen to the carbonyl group stabilizes sulfonium intermediate $\mathbf{3 6}$ and the nucleophile approaches from the side opposite to sulfur with formation predominantly of trans-diastereoisomers, trans-( \pm$)-29 \mathbf{a}$, side trans-( \pm )-33a (Scheme 6).

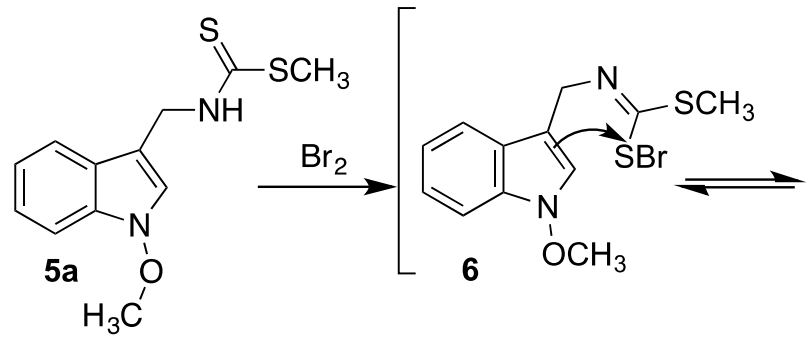<smiles>C=CC1(CBr)N(OC)c2ccccc2C12CN=C(SC)S2</smiles><smiles>CON1CC2(CN=C([SiH3])S2)c2ccccc21</smiles>

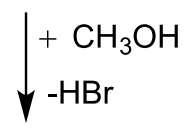<smiles>CO[C@H]1N(OC)c2ccccc2[C@]12CN=C([SiH3])S2</smiles>

trans-( \pm -4a<smiles>C[Se]C(=S)NCc1cn(C(=O)OC(C)(C)C)c2ccccc12</smiles><smiles>CSC1=NCC2(S1)c1ccccc1N(C(=O)OC(C)C)[C@@H]2Br</smiles>
$\downarrow \begin{aligned} & +\mathrm{H}_{2} \mathrm{O} \text { or } \mathrm{CH}_{3} \mathrm{OH} \\ & -\mathrm{HBr}\end{aligned}$<smiles>COC1N(OC)c2ccccc2[C@@]12CN=C([Si])S2</smiles>
cis- $( \pm)-\mathbf{4 b}$<smiles>C[SiH3]C1=NCC2(CCN(C(=O)OC(C)(C)C)C2)c2ccccc21</smiles><smiles>[R]C1N(C(=O)OC(C)(C)C)c2ccccc2[C@]12CN=C([SiH3])S2</smiles> 
Scheme 6. Different mechanisms of the bromine-mediated spirocyclization reactions of 1-methoxybrassinin (5a) and 1-acyl derivatives.

The ratios of diastereoisomers ( \pm )-26a- $( \pm)-33 b$ were determined by the ${ }^{1} \mathrm{H}$ NMR spectra of the crude products after dilution with dichloromethane, washing with brine, drying and evaporation of the solvent. The ratios of diastereoisomers $( \pm)-\mathbf{2 6 a}-( \pm)-\mathbf{3 3 b}$ were determined by integration of well separated signals corresponding to the $\mathrm{H}-2, \mathrm{H}_{\mathrm{a}}$ and $\mathrm{H}_{\mathrm{b}}$ protons. Chromatographic separation of the mixture of diastereoisomers of 1-methoxycarbonylspirobrassinol afforded pure trans-( \pm -28a and pure cis-diastereoisomer cis- $( \pm)-\mathbf{2 8 b}$ as crystalline substances. trans- and cis-Diastereoisomers $\mathbf{3 0 a}, \mathbf{3 0 b}-\mathbf{3 3 a}, \mathbf{3 3 b}$ were separated by column chromatography. In the case of 1-acetylspirobrassinol (26) and 1benzoylspirobrassinol (27), the trans- and cis-diastereoisomers were not separable owing to isomerization during the attempted separation on silica gel. This fact was confirmed by a simple experiment. Prepared products $\mathbf{2 6}$ or $\mathbf{2 7}$ were applied to a TLC plate and the plate was developed. After waiting for one hour, the plate was turned by $90^{\circ}$ and developed again. Detection using UV showed that from each original spot there were now two spots for the two diastereoisomers (Figure 2). The products $\mathbf{2 6}$ and $\mathbf{2 7}$ were isolated as a mixture of trans- and cis-diastereoisomers by column chromatography. It is supposed that diastereoisomers $( \pm)$-26a$( \pm)-26 \mathbf{b}$ and $( \pm)-\mathbf{2 7 a}-( \pm)-27 \mathbf{b}$ isomerize at $\mathrm{C}-2$ atom like the diastereoisomers of 1 methoxyspirobrassinol [trans- $( \pm)-\mathbf{3 a}$, cis- $( \pm)-3 \mathbf{b}]$. In the case of 1-methoxyspirobrassinol (3), isomerization was explained by facile interconversion of hemi-aminal and aminoaldehyde. ${ }^{7}$

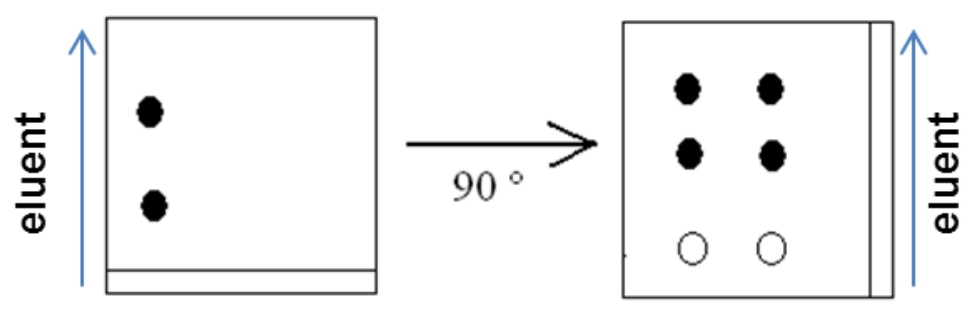

Figure 2. Evidence of isomerization of trans- and cis-diastereoisomers of 1-acetyl-( \pm )-26a$( \pm)-26 \mathbf{b}$ and 1-benzoylspirobrassinol ( \pm$)-27 \mathbf{a}-( \pm)-27 \mathbf{b}$.

The structures of individual diastereoisomers were confirmed by NMR studies, including COSY, HSQC, HMBC and NOESY experiments. The cis-diastereoisomers 7b-11b and 26b33b exhibited in their NOESY spectra a cross peak between $\mathrm{H}-2$ and $\mathrm{H}_{\mathrm{b}}$ protons confirming their cis-configuration. The NOESY specta of structures 7a-11a and 26a-33a did not show the interactions between $\mathrm{H}_{\mathrm{b}}$ and $\mathrm{OH}$ or alkoxy group, which would have confirmed their transdiastereoisomeric structure. However, interactions between $\mathrm{H}-2$ and $\mathrm{H}_{\mathrm{b}}$ were also not observed thus the structures of trans-diastereoisomer was assigned to these products.

Inspection of the ${ }^{1} \mathrm{H}$ NMR spectra of 7-11 and 26-33 revealed a significant difference in the chemical shifts between the H-2 protons of the trans- and cis-diastereoisomers. In all cases the $\delta(\mathrm{H}-2)_{\text {trans }}$ appeared at lower field compared to $\delta(\mathrm{H}-2)_{\text {cis }}$ (Table 6). The higher shielding of $\mathrm{H}-$ 2 in the cis-diastereoisomers is probably caused by anisotropic shielding by the $\mathrm{C}=\mathrm{N}$ double 
bond of the thiazoline ring. This correlation is valid for trans- and cis-diastereoisomers of 1methoxyspirobrassinol (3), ${ }^{7}$ 1-methoxyspirobrassinol methyl ether (4) $)^{7,11}$ and 1-Bocspirobrassinol (29), ${ }^{12}$ in which the diastereoisomeric structures were confirmed by NOE experiments. This consistent chemical shift difference was observed in $\mathrm{CDCl}_{3}$ or DMSO- $d_{6}$. 
Table 6. Chemical shifts of $\mathrm{H}-2$ proton in trans- and cis-diastereoisomers 7a-11b and 26a-33b

\begin{tabular}{|c|c|c|c|c|}
\hline & trans- $\quad \mathrm{R}^{1}$ & & $\mathrm{R}^{1}$ & $\bigcap$ NOESY \\
\hline \multirow{2}{*}{ Compound } & \multirow{2}{*}{$\mathrm{R}^{1}$} & \multirow{2}{*}{$\mathrm{R}^{2}$} & \multirow{2}{*}{ Isomer } & ${ }^{1} \mathrm{H}$ NMR \\
\hline & & & & $\delta(\mathrm{H}-2) \mathrm{ppm}$ \\
\hline$( \pm)-\mathbf{3 a}$ & \multirow{14}{*}{$\mathrm{OCH}_{3}$} & \multirow{2}{*}{$\mathrm{H}$} & $\operatorname{trans} s^{\mathrm{a}}$ & $5.30^{7}$ \\
\hline$( \pm)-\mathbf{3 b}$ & & & $c i s^{\mathrm{a}}$ & $4.80^{7}$ \\
\hline$( \pm)-\mathbf{4 a}$ & & \multirow{2}{*}{$\mathrm{CH}_{3}$} & $\operatorname{trans}^{\mathrm{a}}$ & $4.94^{7}$ \\
\hline$( \pm)-\mathbf{4 b}$ & & & $c i s^{\mathrm{a}}$ & $4.62^{11}$ \\
\hline$( \pm)-7 \mathbf{a}$ & & \multirow{2}{*}{$\mathrm{CH}_{2} \mathrm{CH}_{3}$} & $\operatorname{trans} s^{\mathrm{a}}$ & 5.02 \\
\hline$( \pm)-7 b$ & & & $c i s^{\mathrm{a}}$ & 4.70 \\
\hline$( \pm)-\mathbf{8 a}$ & & \multirow{2}{*}{$\mathrm{CH}\left(\mathrm{CH}_{3}\right)_{2}$} & $\operatorname{trans}^{\mathrm{a}}$ & 5.07 \\
\hline$( \pm)-\mathbf{8 b}$ & & & $c i s^{\mathrm{a}}$ & 4.75 \\
\hline$( \pm)-9 \mathbf{a}$ & & \multirow{2}{*}{$\mathrm{C}\left(\mathrm{CH}_{3}\right)_{3}$} & $\operatorname{trans} s^{\mathrm{a}}$ & 5.26 \\
\hline$( \pm)-9 b$ & & & $c i s^{\mathrm{a}}$ & 4.93 \\
\hline$( \pm)-10 \mathrm{a}$ & & \multirow{2}{*}{$\mathrm{Ph}$} & $\operatorname{trans}^{\mathrm{a}}$ & $5.78^{23}$ \\
\hline$( \pm)-10 b$ & & & $c i s^{\mathrm{a}}$ & $5.49^{23}$ \\
\hline$( \pm)-11 \mathbf{a}$ & & \multirow{2}{*}{ 2-naphthyl } & $\operatorname{trans} s^{\mathrm{a}}$ & 5.96 \\
\hline$( \pm)-\mathbf{1 1 b}$ & & & $c i s^{\mathrm{a}}$ & 5.67 \\
\hline$( \pm)-26 \mathbf{a}$ & \multirow{4}{*}{$\mathrm{COCH}_{3}$} & \multirow{2}{*}{$\mathrm{H}$} & $\operatorname{trans}^{\mathrm{a}}$ & 5.73 \\
\hline$( \pm)-26 b$ & & & $c i s^{\mathrm{a}}$ & 5.42 \\
\hline$( \pm)-30 \mathbf{a}$ & & \multirow{2}{*}{$\mathrm{CH}_{3}$} & $\operatorname{trans}^{\mathrm{a}}$ & 5.41 \\
\hline$( \pm)-\mathbf{3 0 b}$ & & & $c i s^{\mathrm{a}}$ & 5.20 \\
\hline$( \pm)-27 \mathbf{a}$ & \multirow{4}{*}{$\mathrm{COC}_{6} \mathrm{H}_{5}$} & \multirow{2}{*}{$\mathrm{H}$} & $\operatorname{trans}^{\mathrm{a}}$ & 5.95 \\
\hline$( \pm)-27 b$ & & & $c i s^{\mathrm{a}}$ & 5.49 \\
\hline$( \pm)-31 \mathbf{a}$ & & \multirow{2}{*}{$\mathrm{CH}_{3}$} & $\operatorname{trans} s^{\mathrm{a}}$ & 5.52 \\
\hline$( \pm)-31 \mathbf{a}$ & & & $c i s^{\mathrm{a}}$ & 5.22 \\
\hline$( \pm)-28 \mathbf{a}$ & \multirow{4}{*}{$\mathrm{COOCH}_{3}$} & \multirow{2}{*}{$\mathrm{H}$} & $\operatorname{trans}^{\mathrm{a}}$ & 5.95 \\
\hline$( \pm)-\mathbf{2 8 b}$ & & & $c i s^{\mathrm{a}}$ & 5.64 \\
\hline$( \pm)-32 \mathbf{a}$ & & \multirow{2}{*}{$\mathrm{CH}_{3}$} & $\operatorname{trans} s^{\mathrm{a}}$ & 5.56 \\
\hline$( \pm)-\mathbf{3 2} \mathbf{b}$ & & & $c i s^{\mathrm{a}}$ & 5.29 \\
\hline$( \pm)-29 a$ & \multirow{4}{*}{$\mathrm{COOC}\left(\mathrm{CH}_{3}\right)_{3}$} & \multirow{2}{*}{$\mathrm{H}$} & $\operatorname{trans}^{\mathrm{b}}$ & $5.63^{12}$ \\
\hline$( \pm)-29 b$ & & & $c i s^{\mathrm{b}}$ & $5.49^{12}$ \\
\hline$( \pm)-\mathbf{3 3 a}$ & & \multirow{2}{*}{$\mathrm{CH}_{3}$} & trans $s^{\mathrm{b}}$ & $5.42^{31}$ \\
\hline$( \pm)-\mathbf{3 3 b}$ & & & $c i s^{\mathrm{b}}$ & $5.31^{31}$ \\
\hline
\end{tabular}

${ }^{\mathrm{a}} \mathrm{CDCl}_{3} .{ }^{\mathrm{b}} \mathrm{DMSO}-d_{6}$. 
Huggershoff's oxidative bromocyclization of brassinin (5b) and 1-methylbrassinin (37) provided cyclobrassinin (45) or 9-methylcyclobrassinin (46). The formation of cyclobrassinin (45) or 9-methylcyclobrassinin (46) was achieved using various brominating agents (pyridinum tribromide $4534 \%,{ }^{3,32}$ NBS $4535 \%,{ }^{19} 46$ 61\%, ${ }^{33}$ 1,4-dioxane dibromide $4545 \%,{ }^{12} \mathbf{4 6} 40 \%,{ }^{12}$ phenyltrimethylammonium tribromide $\mathbf{4 5} 59 \%^{34}$ ). No comment was made on whether or not these cyclizations afforded spirocyclic structures as minor products.

Therefore we decided to examine the cyclization of brassinin (5b) and 1-methylbrassinin (37) using several cyclization agents $\left(\mathrm{Br}_{2}, \mathrm{DDB}, \mathrm{I}_{2}, \mathrm{NBS}, \mathrm{NCS}, \mathrm{Me}_{3} \mathrm{PhNBr}_{3}\right)$ and solvents (dichloromethane, 1,4-dioxane, methanol). Bromocyclizations of brassinin (5b) and 1methylbrassinin (37) in dichloromethane and 1,4-dioxane with water as a nucleophile did not provide the desired spiroindoline[3,5']thiazoline derivatives $( \pm)-38 \mathbf{a}-( \pm)-\mathbf{3 8 b}$ and $( \pm)-\mathbf{4 1 a}-( \pm)-$ 41b but only unidentified products (Table 7, entries 1,2).

Bromine-mediated cyclization of brassinin (5b) and 1-methylbrassinin (37) in the presence of methanol as a nucleophile led to the the formation of spirobrassinin $[( \pm)-1]$ and 1 methylspirobrassinin $[( \pm)-44]$, respectively (Table 7, entry 11$)$. It is postulated that the initially formed unstable and nonisolable spirobrassinol methyl ether $[( \pm)-39 \mathbf{a},( \pm)-39 \mathbf{b}]$ and 1methylspirobrassinol methyl ether $[( \pm)-\mathbf{4 2 a},( \pm)-\mathbf{4 2} \mathbf{b}]$ undergo oxidation with bromine to provide spirobrassinin $[( \pm)-\mathbf{1}]$ and 1-methylspirobrassinin $[( \pm)-44]$ (Scheme 7). Transformation of brassinin (5b) into spirobrassinin [( \pm -1] was studied with an excess of bromine. The use of 2.2 equivalents of bromine afforded 5-bromospirobrassinin [( \pm -43] in $18 \%$ yield (Table 7 , entry 15). On the basis of the low yield it is assumed that firstly, bromation takes place on the indole core of compounds ( \pm -39a- $( \pm)-39 \mathbf{b}$ at $\mathrm{C}-5$ and subsequently oxidation resulted in the formation of 5-bromospirobrassinin [( \pm -43]. Application of four equivalents of bromine led to an increased yield (Table 7, entry 16). To prevent competitive bromination of the aromatic core, 5-bromobrassin (5c) was used in a cyclization with four equivalents of bromine. 5Bromospirobrassinin [( \pm )-43] was obtained in $64 \%$ yield (Table 7 , entry 19). The proposed mechanism of oxidation of spirobrassinol methyl ether $[( \pm)-39 \mathbf{a},( \pm)-39 \mathbf{b}]$ is depicted in Scheme 8 . 

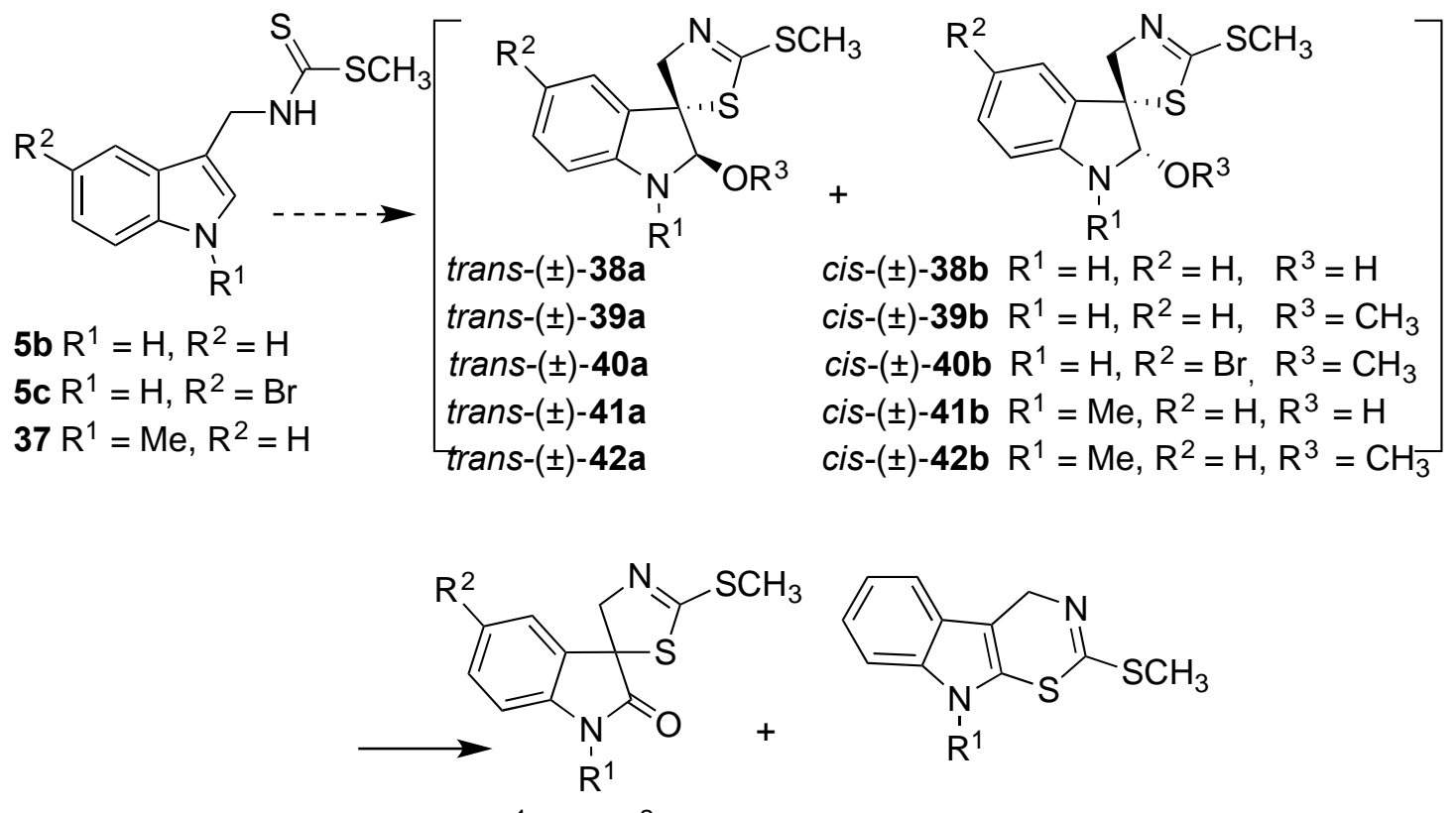

$$
\begin{array}{rlrl}
1 R^{1} & =H, R^{2}=H & & 45 R^{1}=H \\
43 R^{1} & =H, R^{2}=B r & 46 R^{1}=M e \\
44 R^{1} & =M e, R^{2}=H & &
\end{array}
$$

\begin{tabular}{|c|c|c|c|c|c|}
\hline \multirow{3}{*}{ Entry } & \multirow{3}{*}{ Conditions } & \multirow{2}{*}{\multicolumn{2}{|c|}{$\frac{\mathrm{R}^{1}=\mathrm{H}}{\text { Yield }(\%)}$}} & \multirow{2}{*}{\multicolumn{2}{|c|}{$\frac{\mathrm{R}^{1}=\mathrm{CH}_{3}}{\text { Yield }(\%)}$}} \\
\hline & & & & & \\
\hline & & 1 & 45 & 44 & 46 \\
\hline 1 & 1.1eq. $\mathrm{Br}_{2}, \mathrm{CH}_{2} \mathrm{Cl}_{2} / \mathrm{H}_{2} \mathrm{O}, \mathrm{Et}_{3} \mathrm{~N}, \mathrm{rt}$ & decomposition & - & decomposition & - \\
\hline 2 & $\begin{array}{l}\text { 1.1eq. DDB, 1,4-dioxane } / \mathrm{H}_{2} \mathrm{O}, \mathrm{Et}_{3} \mathrm{~N} \text {, } \\
\mathrm{rt}\end{array}$ & decomposition & - & decomposition & - \\
\hline 3 & 1.1eq. $\mathrm{Br}_{2}, \mathrm{MeOH}, \mathrm{Et}_{3} \mathrm{~N}$, rt & 24 & - & 27 & - \\
\hline 4 & 1 eq. $\mathrm{I}_{2}, \mathrm{MeOH}, \mathrm{Et}_{3} \mathrm{~N}, \mathrm{rt}$ & decomposition & - & 30 & - \\
\hline 5 & 1 eq. $\mathrm{Me}_{3} \mathrm{PhNBr}_{3}, \mathrm{MeOH}, \mathrm{Et}_{3} \mathrm{~N}$, rt & 18 & - & 13 & - \\
\hline 6 & 1.1eq. $\mathrm{Br}_{2}, \mathrm{CH}_{2} \mathrm{Cl}_{2} / \mathrm{MeOH}, \mathrm{Et}_{3} \mathrm{~N}$ & 32 & - & 33 & - \\
\hline 7 & $\begin{array}{l}\text { 1.1 eq. } \mathrm{SOCl}_{2}, 1,4 \text {-dioxane/MeOH, } \\
\mathrm{Et}_{3} \mathrm{~N}, \mathrm{rt}\end{array}$ & 16 & - & 25 & - \\
\hline 8 & 1.1eq. NBS, $\mathrm{CH}_{2} \mathrm{Cl}_{2} / \mathrm{MeOH}, \mathrm{Et}_{3} \mathrm{~N}$, rt & 21 & - & 33 & - \\
\hline \multirow[t]{2}{*}{9} & 1.1eq. NCS, $\mathrm{CH}_{2} \mathrm{Cl}_{2} / \mathrm{MeOH}, \mathrm{Et}_{3} \mathrm{~N}$, rt & 40 & - & 40 & - \\
\hline & $\begin{array}{l}\text { 1.1eq. NBS, 1,4-dioxane/MeOH, } \\
\mathrm{Et}_{3} \mathrm{~N}, \mathrm{rt}\end{array}$ & 32 & - & 45 & - \\
\hline 10 & $\begin{array}{l}\text { 1.1eq. NCS, 1,4-dioxane/MeOH, } \\
\mathrm{Et}_{3} \mathrm{~N}, \mathrm{rt}\end{array}$ & 42 & - & 35 & - \\
\hline 11 & $\begin{array}{l}\text { 1.1eq. DDB, 1,4-dioxane/MeOH, } \\
\mathrm{Et}_{3} \mathrm{~N}, \mathrm{rt}\end{array}$ & 47 & - & 55 & - \\
\hline
\end{tabular}

Scheme 7. Bromine-mediated cyclization of brassinin (5b) and 1-methylbrassinin (37).

Table 7. Spirocyclization of brassinin (5b) and 1-methylbrassinin (37): reaction conditions and yields 


\section{Table 7 (continued)}

12

1.1eq. DDB, 1,4-dioxane/EtOH,

$\mathrm{Et}_{3} \mathrm{~N}, \mathrm{rt}$

13

1.1eq. DDB, 1,4-dioxane/i-PrOH,

$\mathrm{Et}_{3} \mathrm{~N}, \mathrm{rt}$

14

1.1eq. DDB, 1,4-dioxane/t-BuOH,

$\mathrm{Et}_{3} \mathrm{~N}, \mathrm{rt}$

15

2.2 eq. DDB, 1,4-dioxane/MeOH,

$\mathrm{Et}_{3} \mathrm{~N}, \mathrm{rt}$

16

4 eq. DDB, 1,4-dioxane/MeOH,

$\mathrm{Et}_{3} \mathrm{~N}, \mathrm{rt}$

17

5c, 1.1eq. DDB, 1,4-dioxane/MeOH,

$\mathrm{Et}_{3} \mathrm{~N}, \mathrm{rt}$

18

5c, 2.2eq. DDB, 1,4-dioxane/MeOH,

$\mathrm{Et}_{3} \mathrm{~N}, \mathrm{rt}$

19
39

68

31

11

65

8

42

60

13

$18(43)$

$49(\mathbf{4 3})$

$64(43)$

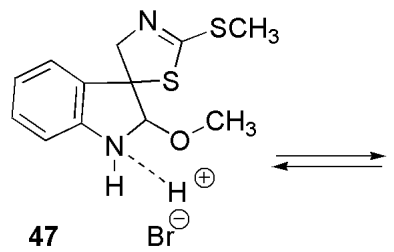

$\mathrm{R}=\mathrm{H}, \mathrm{CH}_{3}$

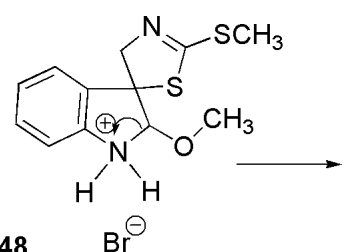

$48 \mathrm{Br}^{\ominus}$

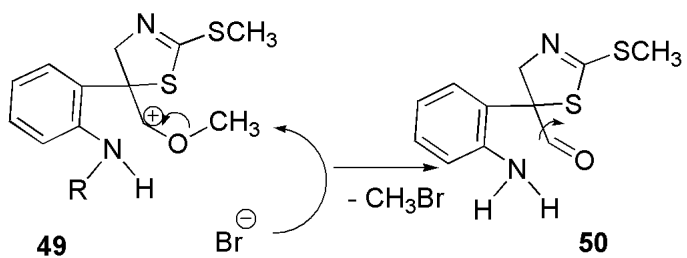

49

50
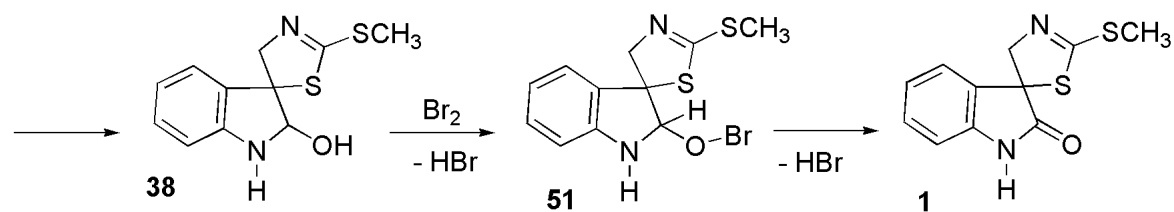

Scheme 8. A plausible reaction mechanism.

The antiproliferative effect (using the colorimetric MTT assay) of the newly synthesized substances was evaluated on six human cancer cell lines; Jurkat (acute T-lymphoblastic leukemia), MCF-7 and MDA-MB-231 (mammary gland adenocarcinomas), HeLa (cervical adenocarcinoma), CEM (acute T-lymphoblastic leukemia) and A-549 (non-small cell lung cancer). $\mathrm{IC}_{50}$ values for the synthesized compounds are presented in Tables 8 and 9. For comparison, Table 8 also includes $\mathrm{IC}_{50}$ values for conventional chemotherapeutic agents (cisplatin and etoposide) and 1-methoxybrassinin (5a), brassinin (5b), 1-Boc-brassinin (12) synthesized previously. 
1-(Methoxycarbonyl)brassinin (25) displayed the highest antiproliferative activity with $\mathrm{IC}_{50}$ from $<10$ to $32.5 \mu \mathrm{mol} \times \mathrm{L}^{-1}$ with the greatest activity in CEM cells (Table 8). 1Benzoylbrassinin (24) reduced the proliferation capacity of CEM cells with $\mathrm{IC}_{50} 25.8 \mu \mathrm{mol} \times$ $\mathrm{L}^{-1}$. 1-Acetylbrassinin (23) did not demonstrate any activity in all the cancer cell lines examined. 1-(Methoxycarbonyl)brassinin (25) and 1-benzoylbrassinin (24) exhibited more significant inhibitory effects than natural phytoalexins 1-methoxybrassinin (5a) and brassinin (5b) against all of the tested cancer lines.

2-Alkoxy analogues of 1-methoxyspirobrassinol methyl ether 7a-11b possess relatively weak antiproliferative activity with $\mathrm{IC}_{50}$ values ranging from 50 to $>100 \mu \mathrm{mol} \times \mathrm{L}^{-1}$ (Table 9). Similar results were obtained with the 1-acyl analogues of 1-methoxyspirobrassinol methyl ether 26a-33b. The highest antiproliferative effects were noted with 1-Boc-spirobrassinol $[( \pm)-$ 29a,,$( \pm)-29 \mathbf{b}]$ and 1-Boc-spirobrassinol methyl ether $[( \pm)-33 \mathbf{a},( \pm)-33 \mathbf{b}]$, where measured $\mathrm{IC}_{50}$ values $29.8-43.4 \mu \mathrm{mol} \times \mathrm{L}^{-1}$ were obtained with leukemic cells (Jurkat and CEM).

Table 8. Antiproliferative activity of 1-methoxybrassinin (5a) and its derivatives

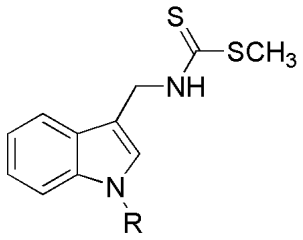

\begin{tabular}{llcccccc}
\hline \multirow{2}{*}{ Compound } & \multirow{2}{*}{} & \multicolumn{5}{c}{ Cancer Cell line, $\mathrm{IC}_{50}\left(\mu \mathrm{mol} \times \mathrm{L}^{-1}\right)$} \\
\cline { 3 - 8 } & & Jurkat & MCF-7 & MDA & HeLa & CEM & A-549 \\
\hline $\mathbf{5} \mathbf{b}^{\mathbf{2 4}}$ & $\mathrm{H}$ & 3100 & $>100$ & $>100$ & $>100$ & 90.2 & $>100$ \\
$\mathbf{5} \mathbf{a}^{\mathbf{2 4}}$ & $\mathrm{OCH}_{3}$ & $>100$ & $>100$ & $>100$ & $>100$ & $>100$ & $>100$ \\
$\mathbf{2 3}$ & $\mathrm{COCH}_{3}$ & 32.4 & 56.1 & 35.2 & 29.0 & 25.8 & 55.2 \\
$\mathbf{2 4}$ & $\mathrm{COC}_{6} \mathrm{H}_{5}$ & 32.5 & 32.5 & 32 & 28.5 & $<10$ & 31.8 \\
$\mathbf{2 5}$ & $\mathrm{COOCH}_{3}$ & 17.8 & 23.0 & 21.4 & 16.9 & 19.6 & 21.4 \\
$\mathbf{1 2}$ & Boc & 12 & 11.4 & 14.7 & 7.7 & 4.4 & 12.2 \\
Cisplatin & & 1.2 & 10.9 & 21.2 & 3.9 & 1.1 & 14.3 \\
Etoposide & & & & & & & \\
\hline
\end{tabular}

The potency of compounds was determined using the MTT (Thiazolyl Blue Tetrazolium Bromide) assay after $72 \mathrm{~h}$ incubation of cells and presented as $\mathrm{IC}_{50}$ (concentration of a given compound that decreased amount of viable cells to $50 \%$ relative to untreated control cells). 
Table 9. Antiproliferative activity of 1-methoxyspirobrassinol methyl ether (4) and its analogues
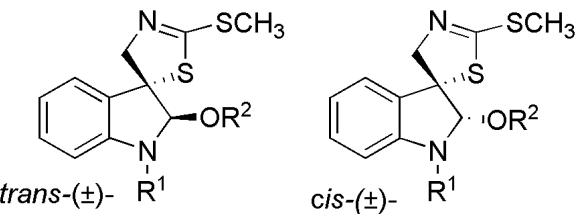

\begin{tabular}{|c|c|c|c|c|c|c|c|c|}
\hline \multirow{2}{*}{ Compound } & \multirow{2}{*}{$\mathrm{R}^{1}$} & \multirow{2}{*}{$\mathrm{R}^{2}$} & \multicolumn{6}{|c|}{ Cancer Cell line, $\mathrm{IC}_{50}\left(\mu \mathrm{mol} \times \mathrm{L}^{-1}\right)$} \\
\hline & & & Jurkat & MCF-7 & MDA & $\mathrm{HeLa}$ & CEM & A-549 \\
\hline $\operatorname{trans}-( \pm)-\mathbf{4} \mathbf{a}^{24}$ & \multirow{12}{*}{$\mathrm{OCH}_{3}$} & \multirow[t]{2}{*}{$\mathrm{CH}_{3}$} & 30.2 & 100 & 100 & 48.9 & 100 & 100 \\
\hline $\operatorname{cis}-( \pm)-\mathbf{4} b^{24}$ & & & 57.4 & 100 & 100 & 53.2 & 100 & 100 \\
\hline trans $-( \pm)-7 \mathbf{a}$ & & \multirow[t]{2}{*}{$\mathrm{CH}_{2} \mathrm{CH}_{3}$} & 70.4 & 85.6 & $>100$ & $>100$ & 83.7 & 85.4 \\
\hline$c i s-( \pm)-7 \mathbf{b}$ & & & $>100$ & $>100$ & $>100$ & $>100$ & $>100$ & $>100$ \\
\hline $\operatorname{trans}-( \pm)-\mathbf{8} \mathbf{a}$ & & \multirow[t]{2}{*}{$\mathrm{CH}\left(\mathrm{CH}_{3}\right)_{2}$} & 73.8 & NT & $>100$ & $>100$ & $>100$ & $>100$ \\
\hline$c i s-( \pm)-\mathbf{8 b}$ & & & $>100$ & NT & $>100$ & $>100$ & $>100$ & $>100$ \\
\hline $\operatorname{trans}-( \pm)-\mathbf{9 a}$ & & \multirow[t]{2}{*}{$\mathrm{C}\left(\mathrm{CH}_{3}\right)_{3}$} & 50.0 & NT & $>100$ & 84.8 & $>100$ & 72.8 \\
\hline$c i s-( \pm)-9 \mathbf{b}$ & & & 59.6 & NT & $>100$ & $>100$ & $>100$ & 68.8 \\
\hline $\operatorname{trans}-( \pm)-\mathbf{1 0} \mathbf{a}^{23}$ & & \multirow[t]{2}{*}{$\mathrm{Ph}$} & 100 & 100 & 100 & 100 & 100 & 100 \\
\hline$c i s-( \pm)-\mathbf{1 0} \mathbf{b}^{23}$ & & & 100 & 100 & 100 & 100 & 100 & 100 \\
\hline trans $-( \pm)-\mathbf{1 1 a}$ & & \multirow[t]{2}{*}{ 2-naphthyl } & $>100$ & $>100$ & $>100$ & $>100$ & $>100$ & $>100$ \\
\hline cis- $( \pm)-\mathbf{1 1 b}$ & & & $>100$ & $>100$ & $>100$ & $>100$ & $>100$ & $>100$ \\
\hline$( \pm)-26 \mathbf{a}, \mathbf{b}$ & \multirow{3}{*}{$\mathrm{COCH}_{3}$} & $\mathrm{H}$ & $>100$ & $>100$ & $>100$ & $>100$ & $>100$ & $>100$ \\
\hline trans $-( \pm)-30 \mathbf{a}$ & & \multirow[t]{2}{*}{$\mathrm{CH}_{3}$} & 49.4 & $>100$ & $>100$ & $>100$ & $>100$ & $>100$ \\
\hline cis- $( \pm)-\mathbf{3 0 b}$ & & & $>100$ & $>100$ & $>100$ & $>100$ & $>100$ & $>100$ \\
\hline$( \pm)-27 \mathbf{a}, \mathbf{b}$ & \multirow{3}{*}{$\mathrm{COC}_{6} \mathrm{H}_{5}$} & $\mathrm{H}$ & 50.0 & $>100$ & $>100$ & $>100$ & $>100$ & $>100$ \\
\hline trans $-( \pm)-31 \mathbf{a}$ & & \multirow[t]{2}{*}{$\mathrm{CH}_{3}$} & 42.0 & $>100$ & $>100$ & 74.0 & 38.0 & $>100$ \\
\hline $\operatorname{cis}-( \pm)-\mathbf{3 1 b}$ & & & 53.0 & 78.0 & $>100$ & 67.0 & 31.0 & 68.0 \\
\hline trans $-( \pm)-\mathbf{2 8 a}$ & \multirow{4}{*}{$\mathrm{COOCH}_{3}$} & \multirow[t]{2}{*}{$\mathrm{H}$} & $>100$ & $>100$ & $>100$ & $>100$ & $>100$ & $>100$ \\
\hline cis- $( \pm)-\mathbf{2 8 b}$ & & & $>100$ & $>100$ & $>100$ & $>100$ & $>100$ & $>100$ \\
\hline trans $-( \pm)-32 \mathbf{a}$ & & \multirow[t]{2}{*}{$\mathrm{CH}_{3}$} & $>100$ & $>100$ & $>100$ & 96.0 & $>100$ & $>100$ \\
\hline cis-( $( \pm)-\mathbf{3 2 b}$ & & & $>100$ & $>100$ & $>100$ & $>100$ & $>100$ & 85.0 \\
\hline trans $-( \pm)-29 \mathbf{a}$ & \multirow{4}{*}{ Boc } & $\mathrm{H}$ & 34.0 & 100 & 82.8 & 78.0 & 30.6 & 100 \\
\hline$c i s-( \pm)-29 b$ & & \multirow{3}{*}{$\mathrm{CH}_{3}$} & 29.8 & 100 & 95.0 & 93.6 & 27.1 & 100 \\
\hline $\operatorname{trans}-( \pm)-33 \mathbf{a}^{24}$ & & & 37.3 & 70.2 & 87.0 & 74.3 & 37.9 & 70.5 \\
\hline$c i s-( \pm)-33 \mathbf{b}^{24}$ & & & 43.4 & 100 & 97.7 & 77.6 & 41.9 & 96.3 \\
\hline
\end{tabular}

The potency of compounds was determined using the MTT (Thiazolyl Blue Tetrazolium Bromide) assay after $72 \mathrm{~h}$ incubation of cells and presented as $\mathrm{IC}_{50}$ (concentration of a given compound that decreased amount of viable cells to $50 \%$ relative to untreated control cells). NT not tested 


\section{Conclusions}

The effect of the solvent and temperature was investigated with the aim of influencing the diastereoselectivity of the bromine-initiated spirocyclization of 1-methoxybrassinin (5a) with methanol. It was found that the use of ether solvents gives rise to a preference for the cisdiastereoisomer cis- $( \pm)-\mathbf{4 b}$, whereas at low temperature the trans-diastereoisomer trans- $( \pm)-\mathbf{4 a}$ is preferred. The bromospirocyclization of brassinin bearing an acyl group (acetyl, benzoyl, methoxycarbonyl and tert-butoxycarbonyl) 12, 23-25 on the indole nitrogen afforded predominantly the trans-diastereoisomer. Bromine-induced spirocyclization reactions of brassinin (5b) and 1-methylbrassinin (37) in the presence of methanol produced spirobrassinin $[( \pm)-1]$ and 1-methylspirobrassinin [( \pm )-44]. The antiproliferative activity of the newly synthesized compounds against selected human cancer cell lines was examined. Substances 25, $( \pm)-29 \mathrm{a},( \pm)-29 \mathrm{~b},( \pm)-33 \mathrm{a},( \pm)-33 \mathrm{~b}$ exhibited the highest inhibitory effects on the growth of CEM cells.

\section{Experimental Section}

General. Melting points were determined on a Koffler hot-stage apparatus and are uncorrected. ${ }^{1} \mathrm{H}$ - and ${ }^{13} \mathrm{C}$-NMR spectra were measured on a Varian Mercury Plus spectrometer $(400 \mathrm{MHz}$ for ${ }^{1} \mathrm{H}$ and $100 \mathrm{MHz}$ for ${ }^{13} \mathrm{C}$ ). Chemical shifts $(\delta)$ are reported in ppm downfield from TMS as the internal standard and the coupling constants $(J)$ are given in Hertz. Microanalyses were performed with a Perkin-Elmer, Model 2400 analyzer. The EI mass spectra were recorded on a GS-MS Trio 1000 (Fisons Instruments) spectrometer at an ionization energy of $70 \mathrm{eV}$. IR spectra were recorded on an IR-75 spectrometer (Zeiss Jena). Flash column chromatography was performed on the Kieselgel Merck Type 9385 at 230-400 mesh. The progress of chemical reactions was monitored by thin layer chromatography (TLC), using Macherey-Nagel plates Alugram ${ }^{\circledR}$ Sil G/UV254. Preparative column chromatography was performed on Kieselgel 60 Merck Type 9385 (0.040-0.063).

\section{Spirocyclization of 1-methoxybrassinin (5a) in the presence of methanol}

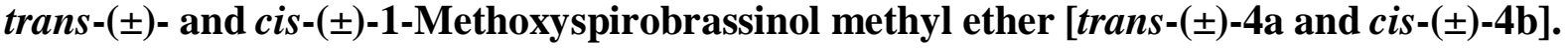
Method A (Table 1 and Table 2): To a stirred solution of 1-methoxybrassinin (5a; $0.027 \mathrm{~g}, 0.1$ $\mathrm{mmol})$ in a mixture of anhydrous solvent/methanol $(0.9 \mathrm{~mL} / 0.1 \mathrm{~mL})$ at $\mathrm{rt}\left(\right.$ or $0{ }^{\circ} \mathrm{C},-20{ }^{\circ} \mathrm{C},-60$ $\left.{ }^{\circ} \mathrm{C}\right)$ was added a freshly prepared solution of $\mathrm{Br}_{2}(0.25 \mathrm{~mL}, 0.11 \mathrm{mmol})$. The stock solution was obtained by dissolving bromine $(0.04 \mathrm{~mL})$ in $1.76 \mathrm{~mL}$ of the used solvent. The reaction mixture was stirred for $15 \mathrm{~min}$, then $\mathrm{Et}_{3} \mathrm{~N}(0.022 \mathrm{~g}, 0.031 \mathrm{~mL}, 0.22 \mathrm{mmol})$ was added. Stirring was continued for $5 \mathrm{~min}$ and the reaction mixture was diluted with $\mathrm{CH}_{2} \mathrm{Cl}_{2}(5 \mathrm{~mL})$ and washed with brine $(2 \times 5 \mathrm{~mL})$. The organic layer was dried over anhydrous $\mathrm{Na}_{2} \mathrm{SO}_{4}$ and the crude product, obtained after evaporation of the solvent, was subjected to ${ }^{1} \mathrm{H}$ NMR spectroscopy to determine the ratio of diastereoisomers trans- $( \pm)-\mathbf{4 a}$ and cis- $( \pm)-\mathbf{4 b}$.

Method B (Table 2, entries 28 and 29): To a stirred solution of 1-methoxybrassinin (5a; 0.027 $\mathrm{g}, 0.1 \mathrm{mmol})$ in dichloromethane $(0.9 \mathrm{~mL})$ at $\mathrm{rt}\left(\right.$ or $\left.-75^{\circ} \mathrm{C}\right)$ was added a freshly prepared 
solution of $\mathrm{Br}_{2}(0.25 \mathrm{~mL}, 0.11 \mathrm{mmol})$. The stock solution was obtained by dissolving bromine $(0.04 \mathrm{~mL})$ in anhydrous $\mathrm{CH}_{2} \mathrm{Cl}_{2}(1.76 \mathrm{~mL})$. The reaction mixture was stirred for $1 \mathrm{~min}$, then methanol $(0.004 \mathrm{~g}, 0.005 \mathrm{~mL}, 0.11 \mathrm{mmol})$ and $\mathrm{Et}_{3} \mathrm{~N}(0.101 \mathrm{~g}, 0.139 \mathrm{~mL}, 1.00 \mathrm{mmol})$ were added. Stirring was continued for $15 \mathrm{~min}$ and the reaction mixture was diluted with $\mathrm{CH}_{2} \mathrm{Cl}_{2}(5$ $\mathrm{mL})$ and washed with brine $(2 \times 5 \mathrm{~mL})$. The organic layer was dried over anhydrous $\mathrm{Na}_{2} \mathrm{SO}_{4}$ and the crude product, obtained after evaporation of the solvent, was subjected to ${ }^{1} \mathrm{H}$ NMR spectroscopy to determine the ratio of diastereoisomers trans-( \pm$)-\mathbf{4 a}$ and cis- $( \pm)-\mathbf{4 b}$.

Method C (Table 3, entry 3): To a stirred mixture of 1-methoxybrassinin (5a; $0.210 \mathrm{~g}, 0.79$ mmol) and powdered molecular sieves $(3 \AA)$ in anhydrous $\mathrm{CH}_{2} \mathrm{Cl}_{2}(4.2 \mathrm{~mL})$ were added powdered anhydrous $\mathrm{K}_{2} \mathrm{CO}_{3}(0.220 \mathrm{~g}, 1.6 \mathrm{mmol})$ and a freshly prepared solution of bromine [2.1 $\mathrm{mL}, 0.9 \mathrm{mmol}$; the stock solution was obtained by dissolving bromine $(0.05 \mathrm{~mL})$ in anhydrous $\left.\mathrm{CH}_{2} \mathrm{Cl}_{2}(2.25 \mathrm{~mL})\right]$. After stirring for $1 \mathrm{~min}$, a freshly prepared solution of complex $\mathrm{CH}_{3} \mathrm{ONa}$-15-crown-5-ether in anhydrous $\mathrm{CH}_{2} \mathrm{Cl}_{2}(1.9 \mathrm{~mL}, 0.90 \mathrm{mmol})$ was added. The stock solution was prepared by dissolving of $\mathrm{CH}_{3} \mathrm{ONa}(0.054 \mathrm{~g} 1.0 \mathrm{mmol})$ in anhydrous $\mathrm{MeOH}(2$ $\mathrm{mL}$ ) with a subsequent addition of 15-crown-5-ether $(0.220 \mathrm{~g}, 0.20 \mathrm{~mL}, 1 \mathrm{mmol})$. MeOH was thoroughly evaporated and the residue was dissolved in anhydrous $\mathrm{CH}_{2} \mathrm{Cl}_{2}(2 \mathrm{~mL})$. Stirring was continued for $10 \mathrm{~min}$, and the reaction mixture was diluted with $\mathrm{CH}_{2} \mathrm{Cl}_{2}(10 \mathrm{~mL})$ and washed with brine $(2 \times 10 \mathrm{~mL})$. The organic layer was dried over anhydrous $\mathrm{Na}_{2} \mathrm{SO}_{4}$. The residue obtained after evaporation of the solvent was subjected to chromatography on $25 \mathrm{~g}$ silica gel ( $n$-hexane/Et $\left.{ }_{2} \mathrm{O} 3: 1\right)$, affording natural diastereoisomer trans- $( \pm)-4 \mathbf{a}(0.086 \mathrm{~g}, 37 \%)$ and unnatural cis-( \pm$)-\mathbf{4 b}(0.026 \mathrm{~g}, 19 \%)$.

The spectral data were identical with those of the natural product trans- $( \pm)-\mathbf{4} \mathbf{a}^{7}$ and unnatural product $\operatorname{cis}-( \pm)-\mathbf{4 b} .^{11}$

\section{Spirocyclization of 1-methoxybrassinin (5a) in the presence of ethanol}

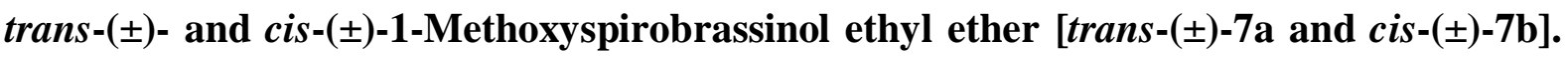
To a stirred solution of 1-methoxybrassinin $(\mathbf{5 a} ; 0.081 \mathrm{~g}, 0.3 \mathrm{mmol})$ in a mixture of anhydrous $\mathrm{CH}_{2} \mathrm{Cl}_{2} / \mathrm{EtOH}(2.7 \mathrm{~mL} / 0.3 \mathrm{~mL})$ at $\mathrm{rt}$ was added a freshly prepared solution of $\mathrm{Br}_{2}(0.77 \mathrm{~mL}$, $0.33 \mathrm{mmol})$. The stock solution was obtained by dissolving of bromine $(0.04 \mathrm{~mL})$ in $1.76 \mathrm{~mL}$ of anhydrous $\mathrm{CH}_{2} \mathrm{Cl}_{2}$. The reaction mixture was stirred for $15 \mathrm{~min}$, then $\mathrm{Et}_{3} \mathrm{~N}(0.067 \mathrm{~g}, 0.09$ $\mathrm{mL}, 0.66 \mathrm{mmol}$ ) was added. Stirring was continued for $5 \mathrm{~min}$ and the reaction mixture was diluted with $\mathrm{CH}_{2} \mathrm{Cl}_{2}(15 \mathrm{~mL})$ and washed with brine $(2 \times 15 \mathrm{~mL})$. The organic layer was dried over anhydrous $\mathrm{Na}_{2} \mathrm{SO}_{4}$. The residue obtained after evaporation of the solvent was subjected to chromatography on silica gel (10 g, $n$-hexane/ $\left.\mathrm{Me}_{2} \mathrm{CO} 5: 1\right)$ and diastereoisomers trans-( \pm 7a, cis- $( \pm)-7 \mathbf{b}$ were separated.

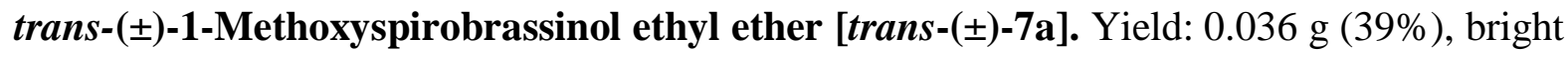
yellow oil, $R_{f} 0.62$ ( $n$-hexane/Me $\mathrm{Me}_{2} \mathrm{CO} 5: 1$ ). Anal. Calcd for $\mathrm{C}_{14} \mathrm{H}_{18} \mathrm{~N}_{2} \mathrm{O}_{2} \mathrm{~S}_{2}$ requires: C, 54.17; H, 5.84; N, 9.02. Found: C, 54.39; H, 6.01; N, 9.23. MS (EI), m/z (\%): $310[\mathrm{M}]^{+}(8), 279$ (83), 251 (30), 117 (100). IR $\left(\mathrm{CHCl}_{3}\right) v_{\max }: 3007,1567(\mathrm{C}=\mathrm{N}), 1460,1380,1193 \mathrm{~cm}^{-1} .{ }^{1} \mathrm{H}$ NMR (400 MHz, $\mathrm{CDCl}_{3}$ ) $\delta$ 7.29-7.22 (m, 2H, H-4, H-6), 7.01 (ddd, J 7.5, J 7.5, J 1.0, 1H, H-5), 6.93 (d, J 7.8, 1H, H-7), $5.02(\mathrm{~s}, 1 \mathrm{H}, \mathrm{H}-2), 4.97\left(\mathrm{~d}, J 15.3,1 \mathrm{H}, \mathrm{H}_{\mathrm{b}}\right), 3.98(\mathrm{dq}, J 9.7, J 7.0,1 \mathrm{H}$, $\left.\underline{\mathrm{CH}_{2}} \mathrm{CH}_{3}\right), 3.95\left(\mathrm{~s}, 3 \mathrm{H}, \mathrm{N}-\mathrm{OCH}_{3}\right), 3.89\left(\mathrm{~d}, J 15.3,1 \mathrm{H}, \mathrm{H}_{\mathrm{a}}\right), 3.82\left(\mathrm{dq}, J 9.7, J 7.0,1 \mathrm{H}, \underline{\mathrm{CH}_{2}} \mathrm{CH}_{3}\right)$, $2.57\left(\mathrm{~s}, 3 \mathrm{H}, \mathrm{SCH}_{3}\right), 1.30\left(\mathrm{t}, J 7.0,3 \mathrm{H}, \mathrm{CH}_{2} \underline{\mathrm{CH}_{3}}\right) .{ }^{13} \mathrm{C} \mathrm{NMR}\left(100 \mathrm{MHz}, \mathrm{CDCl}_{3}\right) \delta 163.4(\mathrm{C}=\mathrm{N})$, 
148.3 (C-7a), 129.8 (C-6), 127.9 (C-3a), 124.1 (C-4), 123.9 (C-5), 113.1 (C-7), 107.8 (C-2), $70.1\left(\mathrm{CH}_{2}\right), 69.1(\mathrm{C}-3), 67.9\left(\mathrm{CH}_{2} \mathrm{CH}_{3}\right), 64.1\left(\mathrm{~N}-\mathrm{OCH}_{3}\right), 15.8\left(\mathrm{CH}_{2} \underline{\mathrm{CH}}_{3}\right), 15.2\left(\mathrm{SCH}_{3}\right)$. NOESY correlations (400 MHz, $\mathrm{CDCl}_{3}$ ): $\mathrm{H}_{\mathrm{a}} / \mathrm{H}_{\mathrm{b}}, \mathrm{H}_{\mathrm{a}} / \mathrm{H}-4, \mathrm{H}-6 / \mathrm{H}-7, \mathrm{H}-4 / \mathrm{H}-5$.

cis-( \pm )-1-Methoxyspirobrassinol ethyl ether [cis-( \pm )-7b]. Yield: $0.027 \mathrm{~g}$ (29\%), bright yellow oil, $R_{f} 0.43$ ( $n$-hexane/Me $\mathrm{MeO}_{2} \mathrm{C}: 1$ ). Anal. Calcd for $\mathrm{C}_{14} \mathrm{H}_{18} \mathrm{~N}_{2} \mathrm{O}_{2} \mathrm{~S}_{2}$ requires: C, 54.17; H, 5.84; N, 9.02. Found: C, 53.86; H, 5.67; N, 9.18. MS of compound cis- $( \pm)-7 \mathbf{b}$ was fully identical with MS of trans- $( \pm)-7 \mathbf{a}$ diastereoisomer. IR $\left(\mathrm{CHCl}_{3}\right) v_{\max }: 3013,1560(\mathrm{C}=\mathrm{N}), 1447$, 1380, $1193 \mathrm{~cm}^{-1} .{ }^{1} \mathrm{H}$ NMR (400 MHz, $\left.\mathrm{CDCl}_{3}\right) \delta$ 7.28-7.24 (m, 2H, H-6, H-4), 7.01 (ddd, J 7.5, $J$ 7.5, J 0.7, 1H, H-5), 6.93 (d, J 7.7, 1H, H-7), 4.70 (s, 1H, H-2), 4.49 (d, J 15.2, 1H, Ha), 4.31 $\left(\mathrm{d}, J 15.2,1 \mathrm{H}, \mathrm{H}_{\mathrm{b}}\right), 3.98$ (dq, J 7.1, J 9.5, 1H, $\left.\underline{\mathrm{CH}}_{2} \mathrm{CH}_{3}\right), 3.95$ (s, $\left.3 \mathrm{H}, \mathrm{N}-\mathrm{OCH}_{3}\right), 3.81$ (dq, J 7.1, $J$ 9.5, $\left.1 \mathrm{H}, \underline{\mathrm{CH}_{2}} \mathrm{CH}_{3}\right), 2.56\left(\mathrm{~s}, 3 \mathrm{H}, \mathrm{SCH}_{3}\right), 1.32\left(\mathrm{t}, J 7.1,3 \mathrm{H}, \mathrm{CH}_{2} \underline{\mathrm{CH}}_{3}\right) .{ }^{13} \mathrm{C} \mathrm{NMR}(100 \mathrm{MHz}$, $\left.\mathrm{CDCl}_{3}\right) \delta 166.9(\mathrm{C}=\mathrm{N}), 147.9(\mathrm{C}-7 \mathrm{a}), 130.1$ (C-6), $128.6(\mathrm{C}-3 \mathrm{a}), 124.1$ (C-5), 123.3 (C-4), 112.9 (C-7), 104.5 (C-2), $73.1\left(\mathrm{CH}_{2}\right), 70.4(\mathrm{C}-3), 67.9\left(\underline{\mathrm{CH}}_{2} \mathrm{CH}_{3}\right), 64.1\left(\mathrm{~N}-\mathrm{OCH}_{3}\right), 15.8$ $\left(\mathrm{CH}_{2} \underline{\mathrm{CH}}_{3}\right), 15.3\left(\mathrm{SCH}_{3}\right)$. NOESY correlations $\left(400 \mathrm{MHz}, \mathrm{CDCl}_{3}\right): \mathrm{H}_{\mathrm{a}} / \mathrm{H}_{\mathrm{b}}, \mathrm{H}-2 / \mathrm{H}_{\mathrm{b}}, \mathrm{H}-6 / \mathrm{H}-7, \mathrm{H}-$ 4/H-5.

\section{Spirocyclization of 1-methoxybrassinin (5a) in the presence of isopropyl alcohol}

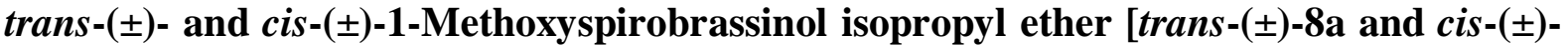
8b]. To a stirred solution of 1-methoxybrassinin $(5 \mathbf{a} ; 0.081 \mathrm{~g}, 0.3 \mathrm{mmol})$ in a mixture of anhydrous $\mathrm{CH}_{2} \mathrm{Cl}_{2} / i-\mathrm{PrOH}(2.7 \mathrm{~mL} / 0.3 \mathrm{~mL})$ at $\mathrm{rt}$ was added a freshly prepared solution of $\mathrm{Br}_{2}$ $(0.77 \mathrm{~mL}, 0.33 \mathrm{mmol})$. The stock solution was obtained by dissolving of bromine $(0.04 \mathrm{~mL})$ in $1.76 \mathrm{~mL}$ of anhydrous $\mathrm{CH}_{2} \mathrm{Cl}_{2}$. The reaction mixture was stirred for $15 \mathrm{~min}$, then $\mathrm{Et}_{3} \mathrm{~N}(0.067$ $\mathrm{g}, 0.09 \mathrm{~mL}, 0.66 \mathrm{mmol}$ ) was added. Stirring was continued for $5 \mathrm{~min}$ and the reaction mixture was diluted with $\mathrm{CH}_{2} \mathrm{Cl}_{2}(15 \mathrm{~mL})$ and washed with brine $(2 \times 15 \mathrm{~mL})$. The organic layer was dried over anhydrous $\mathrm{Na}_{2} \mathrm{SO}_{4}$. The residue obtained after evaporation of the solvent was subjected to chromatography on silica gel (10 g, $n$-hexane/Me $\left.\mathrm{M}_{2} \mathrm{CO} 5: 1\right)$ and diastereoisomers trans- $( \pm)-\mathbf{8} \mathbf{a}$, cis $-( \pm)-\mathbf{8 b}$ were separated.

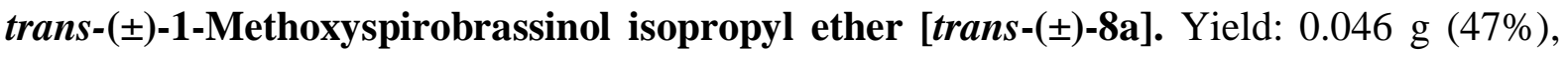
bright yellow oil, $R_{f} 0.59$ ( $n$-hexane/Me $\mathrm{Me}_{2} \mathrm{CO}$ 5:1). Anal. Calcd for $\mathrm{C}_{15} \mathrm{H}_{20} \mathrm{~N}_{2} \mathrm{O}_{2} \mathrm{~S}_{2}$ requires: C, 55.53; H, 6.21; N, 8.63. Found: C, 55.81; H, 6.47; N, 8.35. MS (EI), $m / z(\%): 324[\mathrm{M}]^{+}(7)$, 293 (30), 251 (93), 117 (60), 43 (100). IR $\left(\mathrm{CHCl}_{3}\right) v_{\max }: 2980,1547,1373,1187 \mathrm{~cm}^{-1} .{ }^{1} \mathrm{H}$ NMR $\left(400 \mathrm{MHz}, \mathrm{CDCl}_{3}\right) \delta 7.29$ (d, J 7.7, 1H, H-4), 7.23 (ddd, $J$ 7.7, $J$ 7.7, $\left.J=1.2,1 \mathrm{H}, \mathrm{H}-6\right), 7.00$ (ddd, $J$ 7.7, $J$ 7.7, $J=1.0,1 \mathrm{H}, \mathrm{H}-5$ ), 6.92 (d, J 7.7, 1H, H-7), 5.07 (s, 1H, H-2), 4.99 (d, $J$ 15.2, $\left.1 \mathrm{H}, \mathrm{H}_{\mathrm{b}}\right), 4.06$ [sep, J 6.1, 1H, $\underline{\mathrm{CH}}\left(\mathrm{CH}_{3}\right)_{2}$ ] $, 3.95\left(\mathrm{~s}, 3 \mathrm{H}, \mathrm{N}-\mathrm{OCH}_{3}\right), 3.85\left(\mathrm{~d}, J 15.2,1 \mathrm{H}, \mathrm{H}_{\mathrm{a}}\right), 2.57$ (s, $\left.3 \mathrm{H}, \mathrm{SCH}_{3}\right), 1.31$ [d, $\left.J 6.1,3 \mathrm{H}, \mathrm{CH}\left(\underline{\mathrm{CH}}_{3}\right)_{2}\right], 1.25$ [d, $\left.J 6.1,3 \mathrm{H}, \mathrm{CH}\left(\underline{\mathrm{CH}}_{3}\right)_{2}\right] .{ }^{13} \mathrm{C} \mathrm{NMR}(100$ $\left.\mathrm{MHz}, \mathrm{CDCl}_{3}\right) \delta 163.3(\mathrm{C}=\mathrm{N}), 148.6(\mathrm{C}-7 \mathrm{a}), 129.6(\mathrm{C}-6), 127.9(\mathrm{C}-3 \mathrm{a}), 124.3(\mathrm{C}-4), 123.9(\mathrm{C}-$ 5), 112.5 (C-7), 106.3 (C-2), $76.3\left[\mathrm{CH}\left(\mathrm{CH}_{3}\right)_{2}\right], 70.3\left(\mathrm{CH}_{2}\right), 69.7(\mathrm{C}-3), 64.4\left(\mathrm{~N}-\mathrm{OCH}_{3}\right), 24.1$ and $24.0\left[\mathrm{CH}\left(\underline{\mathrm{CH}}_{3}\right)_{2}\right], 15.2\left(\mathrm{SCH}_{3}\right)$. NOESY correlations $\left(400 \mathrm{MHz}, \mathrm{CDCl}_{3}\right): \mathrm{H}_{\mathrm{a}} / \mathrm{H}_{\mathrm{b}}, \mathrm{H}_{\mathrm{a}} / \mathrm{H}-4$, $\mathrm{OCH}_{3} / \mathrm{CH}\left(\mathrm{CH}_{3}\right)_{2}, \mathrm{H}-6 / \mathrm{H}-7, \mathrm{H}-4 / \mathrm{H}-5$.

cis-( \pm -1-Methoxyspirobrassinol isopropyl ether [cis-( \pm -8b].Yield: $0.029 \mathrm{~g}$ (30\%), bright yellow oil, $R_{f} 0.40$ ( $n$-hexane/ $\mathrm{Me}_{2} \mathrm{CO} 5: 1$ ). Anal. Calcd for $\mathrm{C}_{15} \mathrm{H}_{20} \mathrm{~N}_{2} \mathrm{O}_{2} \mathrm{~S}_{2}$ requires: C, 55.53; H, 6.21; N, 8.63. Found: C, 55.72; H, 5.96; N, 8.85. MS of compound cis- $( \pm)-8 \mathbf{b}$ was fully identical with MS of trans-( \pm -8a diastereoisomer. IR $\left(\mathrm{CHCl}_{3}\right) v_{\max }: 2973,1563,1367,1187$, 
$1106 \mathrm{~cm}^{-1} .{ }^{1} \mathrm{H}$ NMR (400 MHz, $\mathrm{CDCl}_{3}$ ) $\delta$ 7.28-7.24 (m, 2H, H-6, H-4), 7.00 (ddd, J 7.6, J 7.6, $J$ 1.1, 1H, H-5), 6.93 (dd, J 8.2, J 1.0, 1H, H-7), 4.75 (s, 1H, H-2), $4.48\left(\mathrm{~d}, J 15.2,1 \mathrm{H}, \mathrm{H}_{\mathrm{a}}\right.$ ), $4.32\left(\mathrm{~d}, J 15.2,1 \mathrm{H}, \mathrm{H}_{\mathrm{b}}\right), 3.98$ [sep, J 6.1, 1H, $\left.\mathrm{CH}\left(\mathrm{CH}_{3}\right)_{2}\right], 3.94\left(\mathrm{~s}, 3 \mathrm{H}, \mathrm{N}-\mathrm{OCH}_{3}\right), 2.55$ (s, 3H, $\left.\mathrm{SCH}_{3}\right), 1.34\left[\mathrm{~d}, J\right.$ 6.1, 3H, $\left.\mathrm{CH}\left(\underline{\mathrm{CH}}_{3}\right)_{2}\right], 1.26\left[\mathrm{~d}, J 6.1,3 \mathrm{H}, \mathrm{CH}\left(\underline{\mathrm{CH}}_{3}\right)_{2}\right] .{ }^{13} \mathrm{C} \mathrm{NMR}(100 \mathrm{MHz}$, $\left.\mathrm{CDCl}_{3}\right) \delta 167.0(\mathrm{C}=\mathrm{N}), 148.2(\mathrm{C}-7 \mathrm{a}), 130.6(\mathrm{C}-6), 127.7$ (C-3a), 123.8 (C-5), $123.4(\mathrm{C}-4)$, $112.7(\mathrm{C}-7), 103.2(\mathrm{C}-2), 74.1\left[\mathrm{CH}\left(\mathrm{CH}_{3}\right)_{2}\right], 72.8\left(\mathrm{CH}_{2}\right), 70.5(\mathrm{C}-3), 64.2\left(\mathrm{~N}_{-} \mathrm{OCH}_{3}\right), 22.8$ and $22.7\left[\mathrm{CH}\left(\underline{\mathrm{CH}}_{3}\right)_{2}\right], 15.3\left(\mathrm{SCH}_{3}\right)$. NOESY correlations $\left(400 \mathrm{MHz}, \mathrm{CDCl}_{3}\right): \mathrm{H}_{\mathrm{a}} / \mathrm{H}_{\mathrm{b}}, \mathrm{H}-2 / \mathrm{H}_{\mathrm{b}}$, $\underline{\mathrm{CH}}\left(\mathrm{CH}_{3}\right)_{2} / \mathrm{CH}\left(\underline{\mathrm{CH}}_{3}\right)_{2}, \mathrm{H}-6 / \mathrm{H}-7, \mathrm{H}-4 / \mathrm{H}-5$.

\section{Spirocyclization of 1-methoxybrassinin (5a) in the presence of tert-butanol}

trans-( $( \pm)$ - and cis-( \pm$)-1-M e t h o x y s p i r o b r a s s i n o l ~ t e r t-b u t y l$ ether [trans-( \pm$)-9$ a and cis- $( \pm)-$ 9b]. To a stirred solution of 1-methoxybrassinin $(\mathbf{5 a} ; 0.081 \mathrm{~g}, 0.3 \mathrm{mmol})$ in a mixture of anhydrous $\mathrm{CH}_{2} \mathrm{Cl}_{2} / t-\mathrm{BuOH}(2.7 \mathrm{~mL} / 0.3 \mathrm{~mL})$ at $\mathrm{rt}$ was added a freshly prepared solution of $\mathrm{Br}_{2}$ $(0.77 \mathrm{~mL}, 0.33 \mathrm{mmol})$. The stock solution was obtained by dissolving of bromine $(0.04 \mathrm{~mL})$ in $1.76 \mathrm{~mL}$ of anhydrous $\mathrm{CH}_{2} \mathrm{Cl}_{2}$. The reaction mixture was stirred for $15 \mathrm{~min}$, then $\mathrm{Et}_{3} \mathrm{~N}(0.067$ $\mathrm{g}, 0.09 \mathrm{~mL}, 0.66 \mathrm{mmol}$ ) was added. Stirring was continued for $5 \mathrm{~min}$ and the reaction mixture was diluted with $\mathrm{CH}_{2} \mathrm{Cl}_{2}(15 \mathrm{~mL})$ and washed with brine $(2 \times 15 \mathrm{~mL})$. The organic layer was dried over anhydrous $\mathrm{Na}_{2} \mathrm{SO}_{4}$. The residue obtained after evaporation of the solvent was subjected to chromatography on silica gel (10 g, n-hexane/Me $\left.\mathrm{M}_{2} \mathrm{CO} 5: 1\right)$ and diastereoisomers trans- $( \pm)-9 \mathbf{a}$, cis- $( \pm)-\mathbf{9 b}$ were separated.

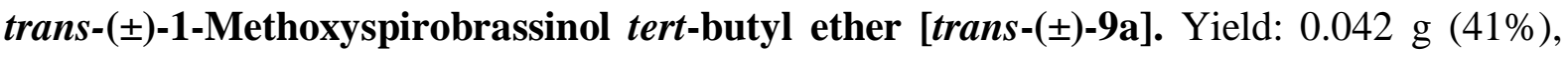
bright yellow oil, $R_{f} 0.64$ ( $n$-hexane/ $\mathrm{Me}_{2} \mathrm{CO} 5: 1$ ). Anal. Calcd for $\mathrm{C}_{16} \mathrm{H}_{22} \mathrm{~N}_{2} \mathrm{O}_{2} \mathrm{~S}_{2}$ requires: C, 56.77; H, 6.55; N, 8.28. Found: C, 56.52; H, 6.74; N, 8.06. MS (EI), m/z (\%): $338[\mathrm{M}]^{+}(2)$, 251 (100), 57 (77). IR $\left(\mathrm{CHCl}_{3}\right) v_{\max }: 3000,1560,1387,1186,1120 \mathrm{~cm}^{-1} .{ }^{1} \mathrm{H} \mathrm{NMR}(400 \mathrm{MHz}$, $\left.\mathrm{CDCl}_{3}\right) \delta$ 7.30-7.28 (m, 1H, H-4), 7.22 (ddd, J 7.5, J 7.5, J 1.2, 1H, H-6), 6.99 (ddd, $J$ 7.5, $J$ 7.5, J 1.0, 1H, H-5), 6.98 (m, 1H, H-7), 5.26 (s, 1H, H-2), 5.07 (d, J 15.2, 1H, Hb), 3.92 (s, 3H, $\left.\mathrm{N}-\mathrm{OCH}_{3}\right), 3.86\left(\mathrm{~d}, J 15.2,1 \mathrm{H}, \mathrm{H}_{\mathrm{a}}\right), 2.53\left(\mathrm{~s}, 3 \mathrm{H}, \mathrm{SCH}_{3}\right), 1.35\left[\mathrm{~s}, 9 \mathrm{H}, \mathrm{C}\left(\mathrm{CH}_{3}\right)_{3}\right] .{ }^{13} \mathrm{C} \mathrm{NMR}(100$ $\left.\mathrm{MHz}, \mathrm{CDCl}_{3}\right) \delta 163.3(\mathrm{C}=\mathrm{N}), 148.6(\mathrm{C}-7 \mathrm{a}), 129.6(\mathrm{C}-6), 127.9(\mathrm{C}-3 \mathrm{a}), 124.3(\mathrm{C}-4), 123.6(\mathrm{C}-$ 5), 112.5 (C-7), $\left.101.0(\mathrm{C}-2), 76.3\left[\mathrm{C}\left(\mathrm{CH}_{3}\right)_{3}\right], 70.3\left(\mathrm{CH}_{2}\right), 69.7(\mathrm{C}-3), 64.4\left(\mathrm{~N}^{-O C H}\right)_{3}\right), 29.3$ $\left[\mathrm{C}\left(\underline{\mathrm{CH}}_{3}\right)_{3}\right], 15.2\left(\mathrm{SCH}_{3}\right)$. NOESY correlations $\left(400 \mathrm{MHz}, \mathrm{CDCl}_{3}\right): \mathrm{H}_{\mathrm{a}} / \mathrm{H}_{\mathrm{b}}, \mathrm{H}_{\mathrm{a}} / \mathrm{H}-4, \mathrm{H}-$ 2/C( $\left.\underline{\mathrm{CH}}_{3}\right)_{3}, \mathrm{H}-6 / \mathrm{H}-7, \mathrm{H}-4 / \mathrm{H}-5$.

cis-( \pm )-1-Methoxyspirobrassinol tert-butyl ether [cis-( \pm )-9b]. Yield: $0.031 \mathrm{~g} \mathrm{(30 \% ),} \mathrm{bright}$ yellow oil, $R_{f} 0.51$ ( $n$-hexane/Me $\mathrm{MeO}_{2} \mathrm{C:1}$ ). Anal. Calcd for $\mathrm{C}_{16} \mathrm{H}_{22} \mathrm{~N}_{2} \mathrm{O}_{2} \mathrm{~S}_{2}$ requires: C, 56.77; H, 6.55; N, 8.28. Found: C, 56.94; H, 6.37; N, 8.12. MS of compound cis-( \pm -9b was fully identical with MS of trans-( \pm -9a diastereoisomer. IR $\left(\mathrm{CHCl}_{3}\right) v_{\max }: 3020,1500,1400,1200$, 913, 720, $660 \mathrm{~cm}^{-1} .{ }^{1} \mathrm{H}$ NMR (400 MHz, $\mathrm{CDCl}_{3}$ ) $\delta$ 7.28-7.23 (m, 2H, H-6, H-4), 7.01-6.97 (m, 1H, H-5), 6.93-6.91 (m, 1H, H-7), 4.93 (s, 1H, H-2), 4.43 (d, J 15.4, 1H, Ha), 4.37 (d, J 15.4, $\left.1 \mathrm{H}, \mathrm{H}_{\mathrm{b}}\right), 3.91\left(\mathrm{~s}, 3 \mathrm{H}, \mathrm{N}-\mathrm{OCH}_{3}\right), 2.54\left(\mathrm{~s}, 3 \mathrm{H}, \mathrm{SCH}_{3}\right), 1.35\left[\mathrm{~s}, 9 \mathrm{H}, \mathrm{C}\left(\mathrm{CH}_{3}\right)_{3}\right] .{ }^{13} \mathrm{C} \mathrm{NMR}(100$ $\left.\mathrm{MHz} \mathrm{CDCl}_{3}\right) \delta 166.8(\mathrm{C}=\mathrm{N}), 148.8(\mathrm{C}-7 \mathrm{a}), 129.9(\mathrm{C}-6), 126.9(\mathrm{C}-3 \mathrm{a}), 123.5(\mathrm{C}-5, \mathrm{C}-4), 112.5$ (C-7), $98.7(\mathrm{C}-2), 76.3\left[\underline{\mathrm{C}}\left(\mathrm{CH}_{3}\right)_{3}\right], 72.4\left(\mathrm{CH}_{2}\right), 71.3(\mathrm{C}-3), 64.3\left(\mathrm{~N}-\mathrm{OCH}_{3}\right), 28.9\left[\mathrm{C}\left(\underline{\mathrm{CH}_{3}}\right)_{3}\right]$, $15.3\left(\mathrm{SCH}_{3}\right)$. NOESY correlations $\left(400 \mathrm{MHz}, \mathrm{CDCl}_{3}\right): \mathrm{H}_{\mathrm{a}} / \mathrm{H}_{\mathrm{b}}, \mathrm{H}-2 / \mathrm{H}_{\mathrm{b}}, \mathrm{H}-2 / \mathrm{C}\left(\underline{\mathrm{CH}}_{3}\right)_{3}, \mathrm{H}-6 / \mathrm{H}-7$, H-4/H-5. 


\section{Spirocyclization of 1-methoxybrassinin (5a) in the presence of naphth-2-ol} trans-( \pm$)$ - and cis-( \pm$)-1-M e t h o x y s p i r o b r a s s i n o l ~ n a p h t h-2-y l ~ e t h e r ~[t r a n s-( \pm)-11 a$ and cis$( \pm)-\mathbf{1 1 b}]$. To a stirred solution of 1-methoxybrassinin $(\mathbf{5 a} ; 0.054 \mathrm{~g}, 0.2 \mathrm{mmol})$ in anhydrous $\mathrm{CH}_{2} \mathrm{Cl}_{2}(3 \mathrm{~mL})$ at $\mathrm{rt}$ was added a freshly prepared solution of $\mathrm{Br}_{2}(0.52 \mathrm{~mL}, 0.22 \mathrm{mmol})$. The stock solution was obtained by dissolving of bromine $(0.04 \mathrm{~mL})$ in $1.76 \mathrm{~mL}$ of anhydrous $\mathrm{CH}_{2} \mathrm{Cl}_{2}$. After stirring for $1 \mathrm{~min}$, the solution of naphth-2-ol $(0.032 \mathrm{~g}, 0.22 \mathrm{mmol})$ and triethylamine $(0.202 \mathrm{~g}, 0.279 \mathrm{~mL}, 2.0 \mathrm{mmol})$ in anhydrous $\mathrm{CH}_{2} \mathrm{Cl}_{2}(3 \mathrm{~mL})$ was added. Stirring was continued for $15 \mathrm{~min}$, then the reaction mixture was diluted with $\mathrm{CH}_{2} \mathrm{Cl}_{2}(10 \mathrm{~mL})$, washed with $1 \mathrm{M} \mathrm{HCl}(5 \mathrm{~mL})$ and brine $(2 \times 10 \mathrm{~mL})$. The organic layer was dried over anhydrous $\mathrm{Na}_{2} \mathrm{SO}_{4}$. The residue obtained after evaporation of the solvent was subjected to chromatography on silica gel (10 g, $n$-hexane/EtOAc 3:1) and diastereoisomers trans-( \pm )-11a, cis- $( \pm)$-11b were separated. trans-Diastereoisomer trans- $( \pm)-11$ a contained small amount of naphth-2-ol as an impurity which was removed by repeated chromatography on silica gel (20 g, $n$-hexane/ $\left.\mathrm{Me}_{2} \mathrm{CO} 1: 1\right)$.

trans-( \pm )-1-Methoxyspirobrassinol naphth-2-yl ether [trans-( \pm )-11a]. Yield: $0.016 \mathrm{~g}(20 \%)$, bright yellow oil, $R_{f} 0.68$ (n-hexane/EtOAc 3:1). Anal. Calcd for $\mathrm{C}_{22} \mathrm{H}_{20} \mathrm{~N}_{2} \mathrm{O}_{2} \mathrm{~S}_{2}$ requires: C, 64.68; H, 4.93; N, 6.86. Found: C, 64.49; H, 4.61; N, 6.61. IR $\left(\mathrm{CHCl}_{3}\right) v_{\max }$ : 3054, 2929, 2847 , 1585, 1462, 1212, 941, $746 \mathrm{~cm}^{-1} .{ }^{1} \mathrm{H}$ NMR (400 MHz, $\left.\mathrm{CDCl}_{3}\right) \delta 7.82-7.75$ (m, 3H, H-arom), 7.65-7.58 (m, 1H, H-arom), 7.48 -7.29 (m, 5H, H-arom), 7.14-7.02 (m, 2H, H-arom), 5.96 (s, $1 \mathrm{H}, \mathrm{H}-2), 5.25\left(\mathrm{~d}, J 15.4,1 \mathrm{H}, \mathrm{H}_{\mathrm{b}}\right), 4.06\left(\mathrm{~d}, J 15.4,1 \mathrm{H}, \mathrm{H}_{\mathrm{a}}\right), 3.91\left(\mathrm{~s}, 3 \mathrm{H}, \mathrm{N}-\mathrm{OCH}_{3}\right), 2.50(\mathrm{~s}, 3 \mathrm{H}$, $\left.\mathrm{SCH}_{3}\right) .{ }^{13} \mathrm{C} \mathrm{NMR}\left(100 \mathrm{MHz}, \mathrm{CDCl}_{3}\right) \delta 163.8(\mathrm{C}=\mathrm{N}), 155.9(\mathrm{C}$-arom), 147.9 (C-arom), 134.3 (C-arom), $129.9(\mathrm{CH}$-arom), $129.6(\mathrm{CH}$-arom), $127.8(\mathrm{C}$-arom), $127.6(\mathrm{CH}$-arom $), 127.2(\mathrm{CH}$ arom), 126.9 (C-arom), 126.4 (CH-arom), 124.5 (CH-arom), 124.0 (CH-arom), 123.9 (CHarom), 119.5 (CH-arom), 113.0 (CH-arom), 112.4 (CH-arom), $107.4(\mathrm{C}-2), 70.3\left(\mathrm{CH}_{2}\right), 69.4$ $(\mathrm{C}-3), 64.1\left(\mathrm{~N}-\mathrm{OCH}_{3}\right), 15.0\left(\mathrm{SCH}_{3}\right)$. NOESY correlations $\left(400 \mathrm{MHz}, \mathrm{CDCl}_{3}\right): \mathrm{H}_{\mathrm{a}} / \mathrm{H}_{\mathrm{b}}$.

cis-( \pm -1-Methoxyspirobrassinol naphth-2-yl ether [cis-( \pm -11b]. Yield: $0.037 \mathrm{~g}(45 \%)$, bright yellow oil, $R_{f} 0.55$ (n-hexane/EtOAc 3:1). Anal. Calcd for $\mathrm{C}_{22} \mathrm{H}_{20} \mathrm{~N}_{2} \mathrm{O}_{2} \mathrm{~S}_{2}$ requires: C, 64.68; H, 4.93; N, 6.86. Found: C, 64.45; H, 4.72; N, 6.58. IR $\left(\mathrm{CHCl}_{3}\right) v_{\max }: 3054,2929,2847$, $1585,1462,1212,941,746 \mathrm{~cm}^{-1} .{ }^{1} \mathrm{H}$ NMR $\left(400 \mathrm{MHz}, \mathrm{CDCl}_{3}\right) \delta$ 7.81-7.68 (m, 3H, H-arom), 7.63-7.56 (m, 1H, H-arom), 7.48 -7.24 (m, 5H, H-arom), 7.11-7.01 (m, 2H, H-arom), 5.67 (s, $1 \mathrm{H}, \mathrm{H}-2), 4.46\left(\mathrm{~d}, J 15.3,1 \mathrm{H}, \mathrm{H}_{\mathrm{a}}\right), 4.35\left(\mathrm{~d}, J 15.3,1 \mathrm{H}, \mathrm{H}_{\mathrm{b}}\right), 3.88\left(\mathrm{~s}, 3 \mathrm{H}, \mathrm{N}-\mathrm{OCH}_{3}\right), 2.53(\mathrm{~s}, 3 \mathrm{H}$, $\left.\mathrm{SCH}_{3}\right) .{ }^{13} \mathrm{C} \mathrm{NMR}\left(100 \mathrm{MHz}, \mathrm{CDCl}_{3}\right) \delta 166.9(\mathrm{C}=\mathrm{N}), 155.9$ (C-arom), 147.3 (C-arom), 134.2 (C-arom), 130.1 (CH-arom), 130.0 (CH-arom), 128.8 (C-arom), $127.7(\mathrm{CH}$-arom), $127.2(\mathrm{CH}-$ arom), 126.5 (CH-arom), 126.3 (C-arom), 124.6 (CH-arom), 124.0 (CH-arom), 123.3 (CHarom), 119.6 (CH-arom), 112.8 (CH-arom), 112.4 (CH-arom), $103.1(\mathrm{C}-2), 72.6\left(\mathrm{CH}_{2}\right), 70.7$ (C-3), $63.9\left(\mathrm{~N}-\mathrm{OCH}_{3}\right), 15.1\left(\mathrm{SCH}_{3}\right)$. NOESY correlations $\left(400 \mathrm{MHz}, \mathrm{CDCl}_{3}\right): \mathrm{H}_{\mathrm{a}} / \mathrm{H}_{\mathrm{b}}, \mathrm{H}-2 / \mathrm{H}_{\mathrm{b}}$,

1-Acetylindole-3-carboxaldehyde (14). To a solution of indole-3-carboxaldehyde $(13 ; 2.90$ $\mathrm{g}, 20.0 \mathrm{mmol})$ in THF $(66 \mathrm{~mL})$ at $0{ }^{\circ} \mathrm{C}$ was added $\mathrm{Ac}_{2} \mathrm{O}(6.12 \mathrm{~g}, 5.6 \mathrm{~mL}, 60.0 \mathrm{mmol})$ and catalytic amount of DMAP. The reaction mixture was stirred for $1 \mathrm{~h}$ at $\mathrm{rt}$. After the reaction was finished, THF was evaporated. The residue was dissolved in $\mathrm{CH}_{2} \mathrm{Cl}_{2}(120 \mathrm{ml})$ and the solution washed with $5 \%$ solution of $\mathrm{KOH}(100 \mathrm{~mL}), 1 \mathrm{M} \mathrm{HCl}(100 \mathrm{ml})$ and $\mathrm{H}_{2} \mathrm{O}(80 \mathrm{ml})$. After drying over anhydrous $\mathrm{Na}_{2} \mathrm{SO}_{4}$ and evaporation of solvent, aldehyde 14 was obtained by 
crystallization from the hot EtOH. Yield: $3.42 \mathrm{~g}(91 \%)$, bright yellow crystals, $R_{f} 0.47$ ( $n$ hexane/Me ${ }_{2} \mathrm{CO} 2: 1$ ), m.p. $165-166{ }^{\circ} \mathrm{C}$ (hot ethanol), lit. ${ }^{35}$ 167-169 ${ }^{\circ} \mathrm{C}$ (n-hexane/EtOAc). Spectral and analytical data are consistent with literature values. ${ }^{35}$

1-Benzoylindole-3-carboxaldehyde (15). To a solution of indole-3-carboxaldehyde (13; 3.0 $\mathrm{g}, 20.0 \mathrm{mmol})$ in THF $(70 \mathrm{~mL})$ at $0{ }^{\circ} \mathrm{C}$ was added $\mathrm{Et}_{3} \mathrm{~N}(10.12 \mathrm{~g}, 14.0 \mathrm{~mL}, 100 \mathrm{mmol})$. The reaction mixture was stirred at $0{ }^{\circ} \mathrm{C}$ for $10 \mathrm{~min}$. After that, $\mathrm{PhCOCl}(3.93 \mathrm{~g}, 3.25 \mathrm{~mL}, 28.0$ mmol) was added and the reaction mixture was stirred at $0{ }^{\circ} \mathrm{C}$ for $45 \mathrm{~min}$. After the reaction was finished, THF was evaporated. The residue obtained after evaporation of the solvent was subjected to column chromatography (30 g silica gel, $n$-hexane/EtOAc 4:1). The obtained compound was further crystallized from $\mathrm{CH}_{2} \mathrm{Cl}_{2} / n$-hexane to afford aldehyde 15. Yield: $4.88 \mathrm{~g}$ (98\%), white crystals, $R_{f} 0.56$ ( $n$-hexane/Me $\mathrm{Me}_{2} \mathrm{CO}$ ), m.p. $68-71^{\circ} \mathrm{C}\left(\mathrm{CH}_{2} \mathrm{Cl}_{2} / n\right.$-hexane). Anal. Calcd for $\mathrm{C}_{16} \mathrm{H}_{11} \mathrm{NO}_{2}$ requires: $\mathrm{C}, 77.10 ; \mathrm{H}, 4.45 ; \mathrm{N}, 5.62$. Found: $\mathrm{C}, 76.77 ; \mathrm{H}, 4.69 ; \mathrm{N}, 5.41$. MS (EI), $m / z(\%): 249[\mathrm{M}]^{+}(43), 105\left[\mathrm{C}_{6} \mathrm{H}_{5} \mathrm{C}=\mathrm{O}\right]^{+}(100), 77\left[\mathrm{C}_{6} \mathrm{H}_{5}\right]^{+}$(79). IR $\left(\mathrm{CHCl}_{3}\right) v_{\max }$ : 3026, $1686(\mathrm{C}=\mathrm{O}), 1673(\mathrm{C}=\mathrm{O}), 1440,706 \mathrm{~cm}^{-1} .{ }^{1} \mathrm{H}$ NMR $\left(400 \mathrm{MHz}, \mathrm{CDCl}_{3}\right) \delta 10.05(\mathrm{~s}, 1 \mathrm{H}$, $\mathrm{CHO}$ ), 8.32-8.30 (m, 1H, H-7), 8.12-8.10 (m, 1H, H-4), 7.94 (s, 1H, H-2) , 7.78-7.76 (m, 2H, H-2’, H-6'), 7.70-7.66 (m, 1H, H-4'), 7.62-7.56 (m, 2H, H-3’' H-5'), 7.49-7.42 (m, 2H, H-5, H-6). ${ }^{13} \mathrm{C}$ NMR (100 MHz, $\left.\mathrm{CDCl}_{3}\right) \delta 185.8(\mathrm{CHO}), 168.5(\mathrm{C}=\mathrm{O}), 137.6(\mathrm{C}-2), 136.8\left(\mathrm{C}-1^{\prime}\right)$, 133.0 (C-4'), 129.4 (C-2’, C-6’), 129.3 (C-7a), 129.0 (C-3’' C-5'), 126.6 (C-6), 126.2 (C-3a), 125.6 (C-5), 122.2 (C-3), 122.0 (C-4), 116.1 (C-7).

1-Methoxycarbonylindole-3-carboxaldehyde (16). To a suspension of $\mathrm{NaH}(2.4 \mathrm{~g}, 60.0$ mmol, 60\% suspension in mineral oil) in anhydrous MeCN (60 mL) was added indole-3carboxaldehyde $(13 ; 2.17 \mathrm{~g}, 15.0 \mathrm{mmol})$. After stirring for $5 \mathrm{~min}$ at $\mathrm{rt}$, methyl chloroformate $(2.83 \mathrm{~g}, 2.3 \mathrm{~mL}, 30.0 \mathrm{mmol})$ was added. The reaction mixture was stirred for $10 \mathrm{~min}$, then poured into cold water $(200 \mathrm{~mL})$ and the product was extracted with EtOAc $(1 \times 150 \mathrm{~mL}$ and $1 \times 100 \mathrm{~mL}$ ). The extract was dried over $\mathrm{Na}_{2} \mathrm{SO}_{4}$. The residue obtained after evaporation of the solvent was crystallized from $\mathrm{CH}_{2} \mathrm{Cl}_{2} / n$-hexane to afford aldehyde 16. Yield: $2.59 \mathrm{~g}(85 \%)$, bright yellow crystals, $R_{f} 0.54$ ( $n$-hexane/Me $\left.{ }_{2} \mathrm{CO} 2: 1\right)$, m.p. $94-96{ }^{\circ} \mathrm{C}\left(\mathrm{CH}_{2} \mathrm{Cl}_{2} / n\right.$-hexane). Anal. Calcd for $\mathrm{C}_{11} \mathrm{H}_{9} \mathrm{NO}_{3}$ requires: $\mathrm{C}, 65.02 ; \mathrm{H}, 4.46$; N, 6.89. Found: $\mathrm{C}, 64.79 ; \mathrm{H}, 4.61 ; \mathrm{N}, 6.73$.

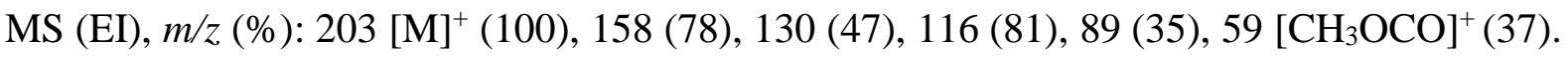
IR $\left(\mathrm{CHCl}_{3}\right) v_{\max }: 3016,1755(\mathrm{C}=\mathrm{O}), 1673(\mathrm{C}=\mathrm{O}), 1440,1345,1226,1096 \mathrm{~cm}^{-1} .{ }^{1} \mathrm{H}$ NMR $(400$ $\left.\mathrm{MHz}, \mathrm{CDCl}_{3}\right) \delta 10.08(\mathrm{~s}, 1 \mathrm{H}, \mathrm{CHO}), 8.28(\mathrm{dd}, J$ 7.3, J 1.4, 1H, H-4), 8.22 (s, 1H, H-2), 8.16 (d, $J 7.3,1 \mathrm{H}, \mathrm{H}-7$ ), 7.43 (ddd, $J 7.3, J 7.3, J 1.4,1 \mathrm{H}, \mathrm{H}-6$ ), 7.38 (ddd, $J 7.3, J 7.3, J 1.1,1 \mathrm{H}$, $\mathrm{H}-5), 4.11\left(\mathrm{~s}, 3 \mathrm{H}, \mathrm{OCH}_{3}\right) .{ }^{13} \mathrm{C} \mathrm{NMR}\left(100 \mathrm{MHz}^{\mathrm{CDCl}} 3\right) \delta 180.9(\mathrm{CHO}), 145.9(\mathrm{C}=\mathrm{O}), 131.2$ (C-2), 131.1 (C-7a), 121.5 (C-6), 121.1 (C-3a), 120.1 (C-5), 117.5 (C-3), 117.4 (C-4), 110.3 (C-7), $49.8\left(\mathrm{CH}_{3}\right)$.

1-Acetylindole-3-carboxaldehyde oxime (17). To a stirred solution of aldehyde $(14 ; 3.42 \mathrm{~g}$, $18.3 \mathrm{mmol})$ in THF $(80 \mathrm{~mL})$ was added a solution of hydroxylammonium chloride $(1.98 \mathrm{~g}$, $28.5 \mathrm{mmol})$ and $\mathrm{NaOAc}(1.72 \mathrm{~g}, 12.6 \mathrm{mmol})$ in water $(14 \mathrm{~mL})$ and the mixture was stirred for $4 \mathrm{~h}$ at rt. After evaporation of THF and addition of water $(80 \mathrm{~mL})$, the oxime 17 was extracted with EtOAc $(1 \times 350 \mathrm{~mL}, 1 \times 250 \mathrm{~mL})$. The extract was dried over $\mathrm{Na}_{2} \mathrm{SO}_{4}$ and the residue obtained after evaporation of the solvent was further crystallized from $\mathrm{Me}_{2} \mathrm{CO} / n$-hexane to afford oxime $\mathbf{1 7}$ as a mixture of $E$ - and $Z$-isomer. 
Yield: $3.26 \mathrm{~g}(88 \%)$, white crystals, $R_{f} 0.44$ (n-hexane/Me $\mathrm{MeO}_{2} \mathrm{CO}$ 2:1), m.p. $145-148{ }^{\circ} \mathrm{C}$ ( $\mathrm{Me}_{2} \mathrm{CO} / n$-hexane). Anal. Calcd for $\mathrm{C}_{11} \mathrm{H}_{10} \mathrm{~N}_{2} \mathrm{O}_{2}$ requires: $\mathrm{C}, 65.34 ; \mathrm{H}, 4.98 ; \mathrm{N}, 13.85$. Found: C, 65.27; H, 4.80; N, 13.51. MS (EI), m/z (\%): $203[\mathrm{M}+\mathrm{H}]^{+}$(7), $202[\mathrm{M}]^{+}$(66), 160 (100), 43 $\left[\mathrm{CH}_{3} \mathrm{CO}\right]^{+}$(78). IR (KBr) v $\max : 3229(\mathrm{OH}) ; 1706(\mathrm{C}=\mathrm{O}) ; 1620(\mathrm{C}=\mathrm{N}) ; 1539 ; 1433 ; 1365 ; 1200$; 1119; 932; $745 \mathrm{~cm}^{-1} .{ }^{1} \mathrm{H}$ NMR (400 MHz, DMSO-d $) \delta 11.64$ (bs, 0.3H, OH min.), 10.73 (bs, 0.7H, OH maj.), 8.60 (s, 0.3H, CH= min.), 8.40 (d, J 8.2, 1H, H-7), 8.26 (s, 0.7H, CH= maj.), 8.14 (d, J 7.6, 1H, H-4), 7.72 (s, 0.3H, H-2 min.), 7.63 (s, 0.7H, H-2 maj.), 7.39-7.34 (m, 1H, $\mathrm{H}-6$ ), 7.31-7.28 (m, 1H, H-5), 2.67 (s, 0.9H, $\mathrm{CH}_{3} \mathrm{~min}$.), 2.64 (s, 2.1H, $\mathrm{CH}_{3}$ maj.). ${ }^{13} \mathrm{C} \mathrm{NMR}$ (100 MHz, DMSO-d6) $\delta 169.3$ (C=O min.), 168.7 (C=O maj.), 143.5 (CH= maj.), 137.3 (C-7a min.), 136.4 (C-7a maj.), 134.8 ( $\mathrm{CH}=\mathrm{min}$.), 130.3 (C-3a min.), 129.0 (C-2 min.), 127.4 (C-3a maj.), 126.8 (C-2 maj.), 126.1 (C-6 maj.), 125.6 (C-6 min.), 124.3 (C-5), 122.6 (C-4), 118.3 (C-7 min.), 116.7 (C-3 maj.), 116.5 (C-7 maj.), 111.7 (C-3 min.), $24.1\left(\mathrm{CH}_{3}\right)$.

1-Benzoylindole-3-carboxaldehyde oxime (18). To a stirred solution of aldehyde (15; $1.0 \mathrm{~g}$, $4.0 \mathrm{mmol})$ in THF $(26 \mathrm{~mL})$ was added a solution of hydroxylammonium chloride $(0.43 \mathrm{~g}, 6.3$ $\mathrm{mmol})$ and $\mathrm{NaOAc}(0.38 \mathrm{~g}, 2.8 \mathrm{mmol})$ in water $(5 \mathrm{~mL})$ and the mixture was stirred for $4 \mathrm{~h}$ at rt. After evaporation of THF and addition of water $(26 \mathrm{~mL})$, the oxime 18 was extracted with EtOAc $(2 \times 80 \mathrm{~mL})$. The extract was dried over $\mathrm{Na}_{2} \mathrm{SO}_{4}$ and the residue obtained after evaporation of the solvent was further crystallized from EtOAc/ $n$-hexane to afford oxime 18 as a mixture of $E$ - and $Z$-isomer. Yield: $0.93 \mathrm{~g}(88 \%)$, bright yellow crystals, $R_{f} 0.46$ ( $n$ hexane/ $\mathrm{Me}_{2} \mathrm{CO} 2: 1$ ), m.p. $115-117{ }^{\circ} \mathrm{C}$ (EtOAc/n-hexane). Anal. Calcd for $\mathrm{C}_{16} \mathrm{H}_{12} \mathrm{~N}_{2} \mathrm{O}_{2}$ requires: C, 72.72; H, 4.58; N, 10.60. Found: C, 72.48; H, 4.90; N, 10.77. MS (EI), $\mathrm{m} / z(\%)$ : $264[\mathrm{M}]^{+}(27), 105\left[\mathrm{C}_{6} \mathrm{H}_{5} \mathrm{C}=\mathrm{O}\right]^{+}(100), 77\left[\mathrm{C}_{6} \mathrm{H}_{5}\right]^{+}(73) . \mathrm{IR}\left(\mathrm{CHCl}_{3}\right)$ v $\max : 3579(\mathrm{OH}), 3020$, $1680(\mathrm{C}=\mathrm{O}), 1446,1339,1165,1125 \mathrm{~cm}^{-1} .{ }^{1} \mathrm{H} \mathrm{NMR}\left(400 \mathrm{MHz}, \mathrm{CDCl}_{3}\right) \delta 11.35(\mathrm{bs}, 0.3 \mathrm{H}, \mathrm{OH}$ $\min$ ), 10.34 (bs, 0.7H, OH maj.), 8.46 (s, 0.3H, CH=N min.), 8.42-8.40 (m, 0.3H, H-7 min), 8.37-8.35 (m, 0.7H, H-7 maj.), 8.21 (s, 0.7H, CH=N maj.), 8.20-8.18 (m, 0.7H, H-4 maj.), 7.787.76 (m, 0.3H, H-4 min.), 7.74-7.72 (m, 2H, H-2’' H-6’), 7.65-7.61 (m, 1H, H-4'), 7.56-7.51 (m, 2H, H-3', H-5') 7.44 (s, 1H, H-2), 7.44-7.39 (m, 1H, H-6), 7.37-7.33 (m, 1H, H-5). ${ }^{13} \mathrm{C}$ NMR (100 MHz, $\left.\mathrm{CDCl}_{3}\right) \delta 168.9$ (C=O min.), 168.3 (C=O maj.), 143.4 (CH=N maj.), 137.3 (C-4’ min.), 136.4 (C-7a maj.), 135.1 (C-7a min.), 134.0 (CH=N min.), 133.8 (C-1' min.), 132.3 (C-1' maj.), 132.2 (C-4' maj.), 129.3 (C-2’' C-6’' min.), 129.1 (C-2', C-6’' maj.), 128.7 (C-3’, C-5’ maj.), 128.6 (C-3’' C-5’ min.), 128.3 (C-2), 127.6 (C-3a), 125.7 (C-6 maj.), 125.3 (C-6 min.), 124.4 (C-5 maj.), 124.2 (C-5 min.), 122.5 (C-4 maj.), 118.2 (C-4 min.), 116.3 (C3 maj.), 116.2 (C-7 min.), 116.1 (C-7 maj.), 111.0 (C-3 min.).

1-Methoxycarbonylindole-3-carboxaldehyde oxime (19). To a stirred solution of aldehyde (16; $2.03 \mathrm{~g}, 10.0 \mathrm{mmol})$ in EtOH (40 mL) was added a solution of hydroxylammonium chloride $(1.04 \mathrm{~g}, 15.0 \mathrm{mmol})$ and $\mathrm{Na}_{2} \mathrm{CO}_{3}(0.73 \mathrm{~g}, 7.0 \mathrm{mmol})$ in water $(5 \mathrm{~mL})$ and the mixture was stirred for $10 \mathrm{~min}$ at rt. After evaporation of $\mathrm{EtOH}$ and addition of water $(10 \mathrm{~mL})$, the oxime 19 was extracted with EtOAc $(1 \times 80 \mathrm{~mL}$ and $1 \times 50 \mathrm{~mL})$. The extract was dried over $\mathrm{Na}_{2} \mathrm{SO}_{4}$ and the residue obtained after evaporation of the solvent was further crystallized from $\mathrm{CH}_{2} \mathrm{Cl}_{2} / n$-hexane to afford oxime 19 as a mixture of $E$ - and $Z$-isomer. Yield: $1.74 \mathrm{~g}(80 \%)$, white crystals, $R_{f} 0.47$ (n-hexane/Me2CO 2:1), m.p. $122-124{ }^{\circ} \mathrm{C}\left(\mathrm{CH}_{2} \mathrm{Cl}_{2} / n\right.$-hexane). Anal. Calcd for $\mathrm{C}_{11} \mathrm{H}_{10} \mathrm{~N}_{2} \mathrm{O}_{3}$ requires: $\mathrm{C}, 60.55 ; \mathrm{H}, 4.62 ; \mathrm{N}, 12.84$. Found: $\mathrm{C}, 60.83 ; \mathrm{H}, 4.37 ; \mathrm{N}, 12.58$. MS (EI), $m / z(\%): 219[\mathrm{M}+\mathrm{H}]^{+}(9), 218[\mathrm{M}]^{+}(90), 175$ (47), 159 (56), 142 (76), 132 (57), 131 
(79), 130 (87), 115 (76), 114 (76), 77 (52), $59\left[\mathrm{CH}_{3} \mathrm{OCO}\right]^{+}(100)$. IR $\left(\mathrm{CHCl}_{3}\right) v_{\max }: 3578(\mathrm{OH})$, $1732(\mathrm{C}=\mathrm{O}), 1433,1345,1246,1082,932 \mathrm{~cm}^{-1} .{ }^{1} \mathrm{H} \mathrm{NMR}\left(400 \mathrm{MHz}, \mathrm{CDCl}_{3}\right) \delta 8.67(\mathrm{~s}, 0.3 \mathrm{H}$, $\mathrm{CH}=\min$.), 8.29 (s, 0.7H, CH= maj.), 8.24 (d, $J 8.1,0.3 \mathrm{H}, \mathrm{H}-7 \mathrm{~min}.), 8.19$ (d, J 8.0, 0.7H, H7 maj.), 8.11 (d, J 7.8, 0.7H, H-4 maj.), 7.79 (s, 0.7 H, H-2 maj.), 7.77 (s, 0.3H, H-2 min.), 7.72 (d, J 7.8, 0.3H, H-4 min.), 7.42-7.31 (m, 2H, H-6, H-5), $4.08\left(\mathrm{~s}, 0.9 \mathrm{H}, \mathrm{OCH}_{3}\right), 4.06(\mathrm{~s}, 2.1 \mathrm{H}$, $\left.\mathrm{OCH}_{3}\right) .{ }^{13} \mathrm{C} \mathrm{NMR}\left(100 \mathrm{MHz}, \mathrm{CDCl}_{3}\right) \delta 151.0(\mathrm{C}=\mathrm{O}), 144.9(\mathrm{CH}=\mathrm{N}$ maj.), 138.8 (C-2 min.), 135.9 (C-7a maj.), 134.4 (C-7a min.), 130.9 (CH=N min.), 128.6 (C-3a min.), 127.5 (C-2 maj.), 126.9 (C-3a maj.), 125.7 (C-6 maj.), 125.2 (C-6 min.), 123.9 (C-5 maj.), 123.6 (C-5 min.), 122.5 (C-4 maj.), 118.3 (C-4 min.), 115.3 (C-7 min.), 115.0 (C-7 maj., C-3 maj.), 109.9 (C-3 min.), 55.2 ( $\mathrm{CH}_{3} \mathrm{O}$ min.), 55.1 ( $\mathrm{CH}_{3} \mathrm{O}$ maj.).

General procedure for the preparation of 1-acyl derivatives of indole-3-ylmethyl amine 20-22.

To a solution of $\mathrm{NiCl}_{2} \cdot 6 \mathrm{H}_{2} \mathrm{O}(1.05 \mathrm{~g}, 4.4 \mathrm{mmol})$ in $\mathrm{MeOH}(40 \mathrm{~mL})$ was added oxime (17-19; $4.0 \mathrm{mmol})$ in $\mathrm{MeOH}(30 \mathrm{~mL})$ followed by $\mathrm{NaBH}_{4}(1.51 \mathrm{~g}, 40.0 \mathrm{mmol})$ in one portion with stirring and cooling with flowing cold water. After $5 \mathrm{~min}, \mathrm{MeOH}$ in the mixture was evaporated to $1 / 4$ of its original volume and mixture was poured into a saturated solution of $\mathrm{NH}_{4} \mathrm{Cl}(250$ $\mathrm{mL})$. After extraction with $\mathrm{CH}_{2} \mathrm{Cl}_{2}$ for compounds $\mathbf{2 0}$ and 21 or EtOAc for compound $22(1 \times$ $150 \mathrm{~mL}, 1 \times 100 \mathrm{~mL}, 2 \times 50 \mathrm{~mL}$ ), drying the extract over $\mathrm{Na}_{2} \mathrm{SO}_{4}$ and evaporation of the solvent, the crude amine 20-22 was obtained. The crude amine 20-22 was employed in the next reaction without purification.

1-(Acetyl)indole-3-ylmethyl amine (20). Following the general procedure, amine 20 was obtained using oxime (17; $0.6 \mathrm{~g}, 3.0 \mathrm{mmol})$.

1-(Benzoyl)indole-3-ylmethyl amine (21). Following the general procedure, amine 21 was obtained using oxime (18; $1.06 \mathrm{~g}, 4.0 \mathrm{mmol})$.

1-(Methoxycarbonyl)indole-3-ylmethyl amine (22). Following the general procedure, amine 22 was obtained using oxime (19;0.87 g, $4.0 \mathrm{mmol})$.

General procedure for the preparation of 1-acetylbrassinin (23) and 1-benzoylbrassinin (24).

To a stirred solution of crude freshly prepared amine $(\mathbf{2 0} ; 0.565 \mathrm{~g}, 3.0 \mathrm{mmol}$ or $\mathbf{2 1} ; 1.00 \mathrm{~g}, 4.0$ mmol) in $\mathrm{CH}_{2} \mathrm{Cl}_{2}(25 \mathrm{~mL}$ or $40 \mathrm{~mL})$ was added $\mathrm{Et}_{3} \mathrm{~N}(0.91 \mathrm{~g}, 1.25 \mathrm{~mL}, 9.0 \mathrm{mmol}$ or $1.21 \mathrm{~g}$, $1.67 \mathrm{~mL}, 12.0 \mathrm{mmol})$ and $\mathrm{CS}_{2}(0.685 \mathrm{~g}, 0.54 \mathrm{~mL}, 9.0 \mathrm{mmol}$ or $0.91 \mathrm{~g}, 0.72 \mathrm{~mL}, 12.0 \mathrm{mmol})$. After stirring for $5 \mathrm{~min}$ at $\mathrm{rt}$, MeI $(1.28 \mathrm{~g}, 0.57 \mathrm{~mL}, 9.0 \mathrm{mmol}$ or $1.70 \mathrm{~g}, 0.75 \mathrm{~mL}, 12.0 \mathrm{mmol})$ was added and stirring was continued for $1 \mathrm{~h}$ or $30 \mathrm{~min}$. The solvent was evaporated and the residue obtained after evaporation of the solvent was subjected to chromatography on silica gel 1-Acetylbrassinin (23). Following the general procedure, product 23 was obtained using of amine $(\mathbf{2 0} ; 0.565 \mathrm{~g}, 3.0 \mathrm{mmol})$ and isolated on silica gel $\left(25 \mathrm{~g}, n\right.$-hexane/Me $\left.\mathrm{MeO}_{2} 2: 1\right)$. The obtained compound was crystallized from $\mathrm{Me}_{2} \mathrm{CO} / n$-hexane to afford 1-acetylbrassinin (23). Yield: $0.651 \mathrm{~g}\left(78 \%\right.$ ), bright yellow crystals, $R_{f} 0.59$ (n-hexane/Me $\mathrm{C}_{2} \mathrm{CO} 2: 1$ ), m.p. $155-156{ }^{\circ} \mathrm{C}$ ( $\mathrm{Me}_{2} \mathrm{CO} / n$-hexane). Anal. Calcd for $\mathrm{C}_{13} \mathrm{H}_{14} \mathrm{~N}_{2} \mathrm{OS}_{2}$ requires: $\mathrm{C}, 56.09 ; \mathrm{H}, 5.07 ; \mathrm{N}, 10.06$. Found: C, 55.72; H, 4.89; N, 10.30. MS (EI), m/z (\%): $279[\mathrm{M}+\mathrm{H}]^{+}(2), 278[\mathrm{M}]^{+}(10), 130(100), 43$ $\left[\mathrm{CH}_{3} \mathrm{CO}\right]^{+}(27)$. IR $\left(\mathrm{CHCl}_{3}\right) v_{\max }: 3366(\mathrm{NH}), 1687(\mathrm{C}=\mathrm{O}), 1440,1373,1120,1080 \mathrm{~cm}^{-1} .{ }^{1} \mathrm{H}$ 
NMR (400 MHz, DMSO-d $\left.d_{6}\right) \delta 9.83$ (bs, $\left.1 \mathrm{H}, \mathrm{NH}\right), 8.38$ (d, $J$ 8.0, 1H, H-7), 7.65 (d, J 7.7, 1H, $\mathrm{H}-4), 7.58$ (s, 1H, H-2), 7.36-7.26 (m, 2H, H-6, H-5), 5.01 (d, J 5.0, 1.8H, $\mathrm{CH}_{2}$ ), 4.74 (d, J 5.4, $\left.0.2 \mathrm{H}, \mathrm{CH}_{2}\right), 2.62\left(\mathrm{~s}, 3 \mathrm{H}, \mathrm{CH}_{3}\right), 2.61\left(\mathrm{~s}, 3 \mathrm{H}, \mathrm{SCH}_{3}\right) .{ }^{13} \mathrm{C}$ NMR $\left(100 \mathrm{MHz}, \mathrm{DMSO}-d_{6}\right) \delta 198.9$ $(\mathrm{C}=\mathrm{S}), 168.8$ (C=O), 135.9 (C-7a), 129.8 (C-3a), 125.5 (C-2), 125.0 (C-6), 123.8 (C-5), 119.6 $(\mathrm{C}-4), 118.0(\mathrm{C}-3), 116.7(\mathrm{C}-7), 42.1\left(\mathrm{CH}_{2}\right), 24.2\left(\mathrm{CH}_{3}\right), 18.1\left(\mathrm{SCH}_{3}\right)$.

1-Benzoylbrassinin (24). Following the general procedure, product $\mathbf{2 4}$ was obtained using of amine $(21 ; 1.00 \mathrm{~g}, 4.0 \mathrm{mmol})$ and isolated on silica gel (60 g, $n$-hexane/EtOAc 2:1). The obtained compound was further crystallized from dichloromethane/ $n$-hexane to afford 1benzoylbrassinin (24). Yield: $0.490 \mathrm{~g}\left(36 \%\right.$ ), bright yellow crystals, $R_{f} 0.66$ ( $n$-hexane/EtOAc 2:1), m.p. $109-111^{\circ} \mathrm{C}\left(\mathrm{CH}_{2} \mathrm{Cl}_{2} / n\right.$-hexane). Anal. Calcd for $\mathrm{C}_{18} \mathrm{H}_{16} \mathrm{~N}_{2} \mathrm{OS}_{2}$ requires: $\mathrm{C}, 63.50 ; \mathrm{H}$, 4.74; N, 8.23. Found: C, 63.21; H, 4.99; N, 8.01. MS (EI), m/z (\%): $340[\mathrm{M}]^{+}(35), 234$ (80), $105\left[\mathrm{C}_{6} \mathrm{H}_{5} \mathrm{C}=\mathrm{O}\right]^{+}(100), 77\left[\mathrm{C}_{6} \mathrm{H}_{5}\right]^{+}(81) . \mathrm{IR}\left(\mathrm{CHCl}_{3}\right) v_{\max }: 3365(\mathrm{NH}), 1679(\mathrm{C}=\mathrm{O}), 1446$, 1352, 1172, $1086 \mathrm{~cm}^{-1} .{ }^{1} \mathrm{H} \mathrm{NMR}\left(400 \mathrm{MHz}, \mathrm{CDCl}_{3}\right) \delta 8.36(\mathrm{~d}, J 8.2,1 \mathrm{H}, \mathrm{H}-7), 7.71-7.69(\mathrm{~m}$, 2H, H-2', H-6'), 7.64-7.59 (m, 2H, H-4, H-4'), 7.55-7.51 (m, 2H, H-3’' H-5'), 7.43-7.39 (m, 1H, H-6), 7.36-7.32 (m, 2H, H-5, H-2), 7.11 (bs, 1H, NH), 5.01 (d, J 4.3, 1.5H, $\mathrm{CH}_{2}$ ), 4.71 (s, $\left.0.5 \mathrm{H}, \mathrm{CH}_{2}\right), 2.71\left(\mathrm{~s}, 0.75 \mathrm{H}, \mathrm{SCH}_{3}\right), 2.63\left(\mathrm{~s}, 2.25 \mathrm{H}, \mathrm{SCH}_{3}\right) .{ }^{13} \mathrm{C} \mathrm{NMR}\left(100 \mathrm{MHz}, \mathrm{CDCl}_{3}\right) \delta$ 199.1 (C=S), 168.4 (C=O), 136.4 (C-7a), 134.1 (C-1'), 132.1 (C-4'), 129.3 (C-3a), 129.1 (C2’, C-6’), 128.7 (C-3’, C-5'), 126.7 (C-2), 125.6 (C-6), 124.2 (C-5), 119.0 (C-4), 116.6 (C-3, $\mathrm{C}-7), 42.3\left(\mathrm{CH}_{2}\right), 18.2\left(\mathrm{SCH}_{3}\right)$.

1-(Methoxycarbonyl)brassinin (25). To a stirred solution of crude freshly prepared amine $(22 ; 0.817 \mathrm{~g}, 4.0 \mathrm{mmol})$ in $\mathrm{MeOH}(25 \mathrm{~mL})$ was added $\mathrm{Et}_{3} \mathrm{~N}(1.21 \mathrm{~g}, 1.67 \mathrm{~mL}, 12.0 \mathrm{mmol})$ and $\mathrm{CS}_{2}(0.91 \mathrm{~g}, 0.72 \mathrm{~mL}, 12.0 \mathrm{mmol})$. After stirring for $5 \mathrm{~min}$ at $\mathrm{rt}$, MeI $(1.70 \mathrm{~g}, 0.75 \mathrm{~mL}, 12.0$ mmol) was added and stirring was continued for $15 \mathrm{~min}$. The solvent was evaporated and the residue obtained after evaporation of the solvent was subjected to chromatography on silica gel (25 g, $n$-hexane/EtOAc 2:1). The obtained compound was further crystallized from $\mathrm{CH}_{2} \mathrm{Cl}_{2} / n$ hexane to afford 1-(methoxycarbonyl)brassinin (25). Yield: $0.683 \mathrm{~g}(58 \%)$, bright yellow crystals, $R_{f} 0.46$ (n-hexane/EtOAc 2:1), m.p. $128-131{ }^{\circ} \mathrm{C}\left(\mathrm{CH}_{2} \mathrm{Cl}_{2} / n\right.$-hexane). Anal. Calcd for $\mathrm{C}_{13} \mathrm{H}_{14} \mathrm{~N}_{2} \mathrm{O}_{2} \mathrm{~S}_{2}$ requires: $\mathrm{C}, 53.04 ; \mathrm{H}, 4.79 ; \mathrm{N}, 9.52$. Found: $\mathrm{C}, 52.81 ; \mathrm{H}, 5.08 ; \mathrm{N}, 9.74$. MS (EI), $m / z$ (\%): $294[\mathrm{M}]^{+}(13), 188(78), 59\left[\mathrm{CH}_{3} \mathrm{OCO}\right]^{+}(100)$. IR $\left(\mathrm{CHCl}_{3}\right) v_{\max }: 3367(\mathrm{NH}), 3020$, $1725(\mathrm{C}=\mathrm{O}), 1439,1371,1276,732 \mathrm{~cm}^{-1} .{ }^{1} \mathrm{H}$ NMR $\left(400 \mathrm{MHz}, \mathrm{CDCl}_{3}\right) \delta 8.15(\mathrm{~d}, J 7.5,1 \mathrm{H}, \mathrm{H}-$ 7), 7.59 (s, 1H, H-2), 7.56 (d, J 7.7, 1H, H-4), 7.39-7.35 (m, 1H, H-6), 7.30-7.27 (m, 1H, H-5), $7.12(\mathrm{~s}, 1 \mathrm{H}, \mathrm{NH}), 5.03\left(\mathrm{~d}, J 4.7,1.7 \mathrm{H}, \mathrm{CH}_{2}\right), 4.74\left(\mathrm{~s}, 0,3 \mathrm{H}, \mathrm{CH}_{2}\right), 4.01\left(\mathrm{~s}, 3 \mathrm{H}, \mathrm{OCH}_{3}\right), 2.73(\mathrm{~s}$, $\left.0.45 \mathrm{H}, \mathrm{SCH}_{3}\right), 2.66\left(\mathrm{~s}, 2.55 \mathrm{H}, \mathrm{SCH}_{3}\right) .{ }^{13} \mathrm{C} \mathrm{NMR}\left(100 \mathrm{MHz}, \mathrm{CDCl}_{3}\right) \delta 199.1(\mathrm{C}=\mathrm{S}), 151.1$ $(\mathrm{C}=\mathrm{O}), 135.5$ (C-7a), 128.9 (C-3a), 125.2 (C-6), 124.5 (C-2), 123.3 (C-5), 119.0 (C-4), 116.3 (C-3), $115.3(\mathrm{C}-7), 53.9\left(\mathrm{OCH}_{3}\right), 42.3\left(\mathrm{CH}_{2}\right), 18.2\left(\mathrm{SCH}_{3}\right)$.

General procedure for the spirocyclization of 1-acyl derivatives of brassinin 23-25 with bromine in the presence of water. To a stirred solution of 1-acyl derivatives of brassinin 23$25(0.5 \mathrm{mmol})$ in a mixture of $\mathrm{CH}_{2} \mathrm{Cl}_{2} /$ water $(3.6 \mathrm{~mL} / 0.4 \mathrm{~mL})$ at $\mathrm{rt}$ was added freshly prepared solution of $\mathrm{Br}_{2}(1.26 \mathrm{~mL}, 0.55 \mathrm{mmol})$. The stock solution was obtained by dissolving of 0.04 $\mathrm{mL}$ of bromine in $1.76 \mathrm{~mL}$ of anhydrous $\mathrm{CH}_{2} \mathrm{Cl}_{2}$. The reaction mixture was stirred for $15 \mathrm{~min}$, then $\mathrm{Et}_{3} \mathrm{~N}(0.111 \mathrm{~g}, 0.15 \mathrm{~mL}, 1.1 \mathrm{mmol})$ was added. Stirring was continued for $5 \mathrm{~min}$ and the reaction mixture was diluted with $\mathrm{CH}_{2} \mathrm{Cl}_{2}(25 \mathrm{~mL})$ and washed with brine $(2 \times 25 \mathrm{~mL})$. The 
organic layer was dried over anhydrous $\mathrm{Na}_{2} \mathrm{SO}_{4}$ and the residue obtained after evaporation of the solvent subjected to chromatography.

trans-( $( \pm)-$ and cis- $( \pm)-1$-Acetylspirobrassinol [trans $-( \pm)-26$ a and cis $-( \pm)-26 b]$. Following the general procedure, products trans- $( \pm)-26 \mathbf{a}$ and cis- $( \pm)-26 \mathbf{b}$ were obtained using $0.139 \mathrm{~g}(0.5$ $\mathrm{mmol})$ of 1-acetylbrassinin (23) and isolated on silica gel (15 g, $n$-hexane/EtOAc 1:3) as

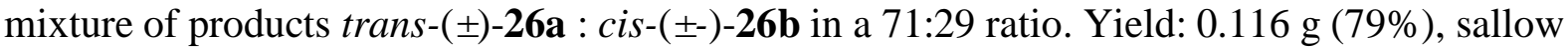
oil, $R_{f}$ (trans) 0.52 ( $n$-hexane/EtOAc 1:3), $R_{f}$ (cis) 0.37 ( $n$-hexane/EtOAc 1:3). Anal. Calcd for $\mathrm{C}_{13} \mathrm{H}_{14} \mathrm{~N}_{2} \mathrm{O}_{2} \mathrm{~S}_{2}$ requires: C, 53.04; $\mathrm{H}, 4.79 ; \mathrm{N}, 9.52$. Found: $\mathrm{C}, 53.31 ; \mathrm{H}, 4.50 ; \mathrm{N}, 9.83$. MS (EI), $m / z$ (\%): $295[\mathrm{M}+\mathrm{H}]^{+}(9), 294[\mathrm{M}]^{+}(47), 251(50), 43\left[\mathrm{CH}_{3} \mathrm{CO}\right]^{+}(100) . \mathrm{IR}\left(\mathrm{CHCl}_{3}\right) v_{\max }: 3279$ $(\mathrm{OH}), 3013,1649(\mathrm{C}=\mathrm{O}), 1547(\mathrm{C}=\mathrm{N}), 1466,1378,1099 \mathrm{~cm}^{-1} .{ }^{1} \mathrm{H}$ NMR $\left(400 \mathrm{MHz}, \mathrm{CDCl}_{3}\right) \delta$ $8.06(\mathrm{~d}, J$ 7.6, 0.7H, H-7 trans), 7.82 (d, J 7.6, 0.3H, H-7 cis), 7.43 (dd, $J$ 7.5, J 0.6, 0.7H, H-4 trans), 7.36 (d, J 7.4, 0.3H, H-4 cis), 7.32-7.27 (m, 1H H-6), 7.11 (ddd, J 0.9, J 7.5, J 7.5, 1H, H-5), 6.11 (s, 0.3H, OH cis), 5.73 (s, 0.7H, H-2 trans), 5.42 (s, 0.3H, H-2 cis), 5.17 (s, 0.7H, $\mathrm{OH}$ trans), 4.95 (d, J 15.6, 0.7H, $\mathrm{H}_{\mathrm{b}}$ trans), 4.35 (d, J 15.3, 0.3H, $\mathrm{H}_{\mathrm{b}}$ cis $), 4.31(\mathrm{~d}, J 15.6,0.7 \mathrm{H}$, $\mathrm{H}_{\mathrm{a}}$ trans), $3.93\left(\mathrm{~d}, J 15.3,0.3 \mathrm{H}, \mathrm{H}_{\mathrm{a}}\right.$ cis $), 2.57$ (s, 2.1H, $\mathrm{SCH}_{3}$ trans $), 2.54$ (s, 0.9H, $\mathrm{SCH}_{3}$ cis), 2.39 (s, 3H, $\mathrm{CH}_{3}$ cis, trans). ${ }^{13} \mathrm{C} \mathrm{NMR}\left(100 \mathrm{MHz}, \mathrm{CDCl}_{3}\right) \delta 170.1(\mathrm{C}=\mathrm{O}), 166.2(\mathrm{C}=\mathrm{N}), 141.4$ (C-7a), 130.0 (C-6), 128.5 (C-3a), 124.7 (C-5), 123.9 (C-4), 117.2 (C-7 trans), 114.1 (C-7 cis), 93.0 (C-2 cis), 88.5 (C-2 trans), $75.1\left(\mathrm{CH}_{2}\right.$ cis $), 71.5$ (C-3 trans), $67.6(\mathrm{C}-3$ cis $), 66.3\left(\mathrm{CH}_{2}\right.$ trans), $23.3\left(\mathrm{CH}_{3}\right), 15.2\left(\mathrm{SCH}_{3}\right)$. NOESY correlations $\left(400 \mathrm{MHz}, \mathrm{CDCl}_{3}\right): \mathrm{H}_{\mathrm{a}} / \mathrm{H}-4$ (trans), $\mathrm{H}-$ 2/Hb $(c i s), \mathrm{H}-5 / \mathrm{H}-4, \mathrm{H}-6 / \mathrm{H}-7$.

trans-( $( \pm)-$ and cis-( \pm$)-1-B e n z o y l s p i r o b r a s s i n o l ~[t r a n s-( \pm)-27$ a and cis $-( \pm)-27 b]$. Following the general procedure, products trans- $( \pm)-27 \mathbf{a}$ and cis- $( \pm)-27 \mathbf{b}$ were obtained using $0.170 \mathrm{~g}(0.5$ mmol) of 1-benzoylbrassinin (24) and isolated on silica gel (15 g, $\left.\mathrm{CH}_{2} \mathrm{Cl}_{2} / \mathrm{Me}_{2} \mathrm{CO} 8: 1\right)$ as a mixture of products trans- $( \pm)-27:$ cis- $( \pm-)-27 b$ in a $64: 36$ ratio. Yield: $0.137 \mathrm{~g}(77 \%)$, sallow oil, $R_{f}$ (trans) $0.67\left(\mathrm{CH}_{2} \mathrm{Cl}_{2} / \mathrm{Me}_{2} \mathrm{CO} 8: 1\right), R_{f}$ (cis) $0.43\left(\mathrm{CH}_{2} \mathrm{Cl}_{2} / \mathrm{Me}_{2} \mathrm{CO} 8: 1\right)$. Anal. Calcd for $\mathrm{C}_{18} \mathrm{H}_{16} \mathrm{~N}_{2} \mathrm{O}_{2} \mathrm{~S}_{2}$ requires: $\mathrm{C}, 60.65 ; \mathrm{H}, 4.52 ; \mathrm{N}, 7.86$. Found: $\mathrm{C}, 60.31 ; \mathrm{H}, 4.79 ; \mathrm{N}, 7.53$. MS (EI), $m / z(\%): 357[\mathrm{M}+\mathrm{H}]^{+}(11), 356[\mathrm{M}]^{+}(35), 251(71), 105\left[\mathrm{C}_{6} \mathrm{H}_{5} \mathrm{C}=\mathrm{O}\right]^{+}(100), 77\left[\mathrm{C}_{6} \mathrm{H}_{5}\right]^{+}(92)$. IR $\left(\mathrm{CHCl}_{3}\right) v_{\max }: 3365(\mathrm{OH}), 3006,1666(\mathrm{C}=\mathrm{O}), 1560(\mathrm{C}=\mathrm{N}), 1466,1365,1086,939 \mathrm{~cm}^{-1} .{ }^{1} \mathrm{H}$ NMR (400 MHz, $\left.\mathrm{CDCl}_{3}\right) \delta 7.70$ (d, J 7.1, 0.6 H, H-2’' H-6' cis), 7.62 (d, J 7.5, 1.4 H, H-2', H-6’ trans), 7.56-7.53 (m, 1H, H-4'), 7.48-7.38 (m, 4H, H-7, H-4, H-3’' H-5’), 7.22-7.05 (m, 2H, H-6, H-5), 5.95 (s, 0.7H, H-2 trans), 5.49 (s, 0.3H, H-2 cis), 4.97 (d, J 15.6, 0.7H, $\mathrm{H}_{\mathrm{b}}$ trans), $4.63\left(\mathrm{~s}, 1 \mathrm{H}, \mathrm{OH}\right.$ cis, trans), $4.37\left(\mathrm{~d}, J 15.2,0.3 \mathrm{H}, \mathrm{H}_{\mathrm{b}}\right.$ cis $), 4.34\left(\mathrm{~d}, J 15.6,0.7 \mathrm{H}, \mathrm{H}_{\mathrm{a}}\right.$ trans), $3.98\left(\mathrm{~d}, J 15.2,0.3 \mathrm{H}, \mathrm{H}_{\mathrm{a}}\right.$ cis), $2.55\left(\mathrm{~s}, 3 \mathrm{H}, \mathrm{SCH}_{3}\right) .{ }^{13} \mathrm{C} \mathrm{NMR}\left(100 \mathrm{MHz}, \mathrm{CDCl}_{3}\right) \delta 170.2$ $(\mathrm{C}=\mathrm{O}$ trans $), 170.1(\mathrm{C}=\mathrm{O}$ cis $), 165.9(\mathrm{C}=\mathrm{N}$ cis $), 164.7(\mathrm{C}=\mathrm{N}$ trans $), 140.5(\mathrm{C}-7 \mathrm{a}$ trans $), 140.0$ (C-7a cis), 135.1 (C-1' cis), 134.8 (C-1'trans), 131.6 (C-4'trans), 131.4 (C-4'cis), 131.2 (C3a), 129.5 (C-6 trans), 129.4 (C-6 cis), 128.7 (C-3', C-5'trans), 128.6 (C-3', C-5' cis), 127.9 (C-2', C-6'trans), 127.8 (C-2', C-6' cis), 125.0 (C-5 cis), 124.4 (C-5 trans), 124.3 (C-4 cis), 124.1 (C-4 trans), 117.0 (C-7 cis), 116.1 (C-7 trans), 92.7 (C-2 trans), 89.2 (C-2 cis), 74.1 $\left(\mathrm{CH}_{2}\right.$ cis $), 70.4$ (C-3 trans), 66.7 ( $\mathrm{CH}_{2}$ trans), 64.3 (C-3 cis), $15.2\left(\mathrm{SCH}_{3}\right.$ trans $), 15.1\left(\mathrm{SCH}_{3}\right.$ cis). NOESY correlations $\left(400 \mathrm{MHz}, \mathrm{CDCl}_{3}\right): \mathrm{H}_{\mathrm{a}} / \mathrm{H}-4$ (trans), $\mathrm{H}_{\mathrm{a}} / \mathrm{H}_{\mathrm{b}}$ (trans), $\mathrm{H}-2 / \mathrm{H}_{\mathrm{b}}($ cis $)$, $\mathrm{H}_{\mathrm{a}} / \mathrm{H}_{\mathrm{b}}($ cis $)$.

trans-( \pm$)$ - and cis-( \pm$)-1-M e t h o x y c a r b o n y l s p i r o b r a s s i n o l ~[t r a n s-( \pm)-28 a$ and cis-( \pm$)-28 b]$. Following the general procedure, products trans-( \pm$)-\mathbf{2 8 a}$ and $c i s-( \pm)-\mathbf{2 8 b}$ were obtained using 
$0.147 \mathrm{~g}(0.5 \mathrm{mmol})$ of 1-(methoxycarbonyl)brassinin (25) and separated on silica gel (30 g, $\left.\mathrm{CH}_{2} \mathrm{Cl}_{2} / \mathrm{Me}_{2} \mathrm{CO} 9: 1\right)$. Both diastereoisomers trans-( \pm$)-\mathbf{2 8 a}$ and cis- $( \pm)-\mathbf{2 8 b}$ were crystallized from $\mathrm{CH}_{2} \mathrm{Cl}_{2} / n$-hexane.

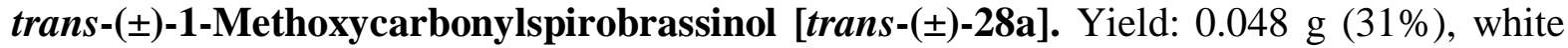
crystals, $R_{f} 0.48\left(\mathrm{CH}_{2} \mathrm{Cl}_{2} / \mathrm{Me}_{2} \mathrm{CO} 9: 1\right)$, mp 135-138 ${ }^{\circ} \mathrm{C}\left(\mathrm{CH}_{2} \mathrm{Cl}_{2} / n\right.$-hexane $)$. Anal. Calcd for $\mathrm{C}_{13} \mathrm{H}_{14} \mathrm{~N}_{2} \mathrm{O}_{3} \mathrm{~S}_{2}$ requires: C, 50.30; H, 4.55; N, 9.03. Found: $\mathrm{C}, 50.49 ; \mathrm{H}, 4.37 ; \mathrm{N}, 8.85$. MS (EI), $m / z(\%): 311[\mathrm{M}+\mathrm{H}]^{+}(16), 310[\mathrm{M}]^{+}(65), 203$ (100), 159 (87), 117 (47), 87 (87), 72 (63), 59 $\left[\mathrm{CH}_{3} \mathrm{OCO}^{+}(58) . \mathrm{IR}\left(\mathrm{CHCl}_{3}\right) v_{\max }: 3567(\mathrm{OH}), 3099,1699(\mathrm{C}=\mathrm{O}), 1547(\mathrm{C}=\mathrm{N}), 1433,1073\right.$ $\mathrm{cm}^{-1} .{ }^{1} \mathrm{H}$ NMR $\left(400 \mathrm{MHz}, \mathrm{CDCl}_{3}\right) \delta 7.80(\mathrm{bs}, 1 \mathrm{H}, \mathrm{H}-7), 7.38(\mathrm{~d}, J 7.5,1 \mathrm{H}, \mathrm{H}-4), 7.31-7.26$ (m, 1H, H-6), 7.10-7.06 (m, 1H, H-5), 5.95 (s, 1H, H-2), 5.04 (d, J 15.5, 1H, Hb) 4.64 (bs, 1H, $\mathrm{OH}), 4.31\left(\mathrm{~d}, J 15.5,1 \mathrm{H}, \mathrm{H}_{\mathrm{a}}\right), 3.91\left(\mathrm{~s}, 3 \mathrm{H}, \mathrm{OCH}_{3}\right), 2.55\left(\mathrm{~s}, 3 \mathrm{H}, \mathrm{SCH}_{3}\right) .{ }^{13} \mathrm{C} \mathrm{NMR}(100 \mathrm{MHz}$, $\left.\mathrm{CDCl}_{3}\right) \delta 164.8(\mathrm{C}=\mathrm{N}), 153.8(\mathrm{C}=\mathrm{O}), 140.6(\mathrm{C}-7 \mathrm{a}), 129.9(\mathrm{C}-6), 129.8(\mathrm{C}-3 \mathrm{a}), 123.9(\mathrm{C}-4, \mathrm{C}-$ 5), 114.9 (C-7), $91.6(\mathrm{C}-2), 70.6(\mathrm{C}-3), 67.2\left(\mathrm{CH}_{2}\right), 53.2\left(\mathrm{OCH}_{3}\right), 15.2\left(\mathrm{SCH}_{3}\right)$. NOESY correlations (400 MHz, $\mathrm{CDCl}_{3}$ ): $\mathrm{H}_{\mathrm{a}} / \mathrm{H}_{\mathrm{b}}, \mathrm{H}_{\mathrm{a}} / \mathrm{H}-4, \mathrm{H}-4 / \mathrm{H}-5, \mathrm{H}-5 / \mathrm{H}-6, \mathrm{H}-6 / \mathrm{H}-7$.

cis-( \pm -1-Methoxycarbonylspirobrassinol [cis-( \pm )-28b]. Yield: $0.011 \mathrm{~g}(7 \%)$, white crystals, $R_{f} 0.57 \quad\left(\mathrm{CH}_{2} \mathrm{Cl}_{2} / \mathrm{Me}_{2} \mathrm{CO}\right.$ 9:1), mp 129-132 ${ }^{\circ} \mathrm{C} \quad\left(\mathrm{CH}_{2} \mathrm{Cl}_{2} / n\right.$-hexane $)$. Anal. Calcd for $\mathrm{C}_{13} \mathrm{H}_{14} \mathrm{~N}_{2} \mathrm{O}_{3} \mathrm{~S}_{2}$ requires: C, 50.30; H, 4.55; N, 9.03. Found: C, 50.58; H, 4.39; N, 9.31. MS of compound cis-( \pm -28b was fully identical with MS of trans- $( \pm)-28 \mathbf{a}$ diastereoisomer. IR $\left(\mathrm{CHCl}_{3}\right) v_{\max }: 3526(\mathrm{OH}), 3132,1706(\mathrm{C}=\mathrm{O}), 1567(\mathrm{C}=\mathrm{N}), 1476,1378,1083 \mathrm{~cm}^{-1} .{ }^{1} \mathrm{H} \mathrm{NMR}$ $\left(400 \mathrm{MHz}, \mathrm{CDCl}_{3}\right) \delta 7.68$ (bs, 1H, H-7), 7.39 (d, J 7.5, 1H, H-4), 7.31-7.26 (m, 1H, H-6), 7.107.06 (m, 1H, H-5), 6.11 (bs, 1H, OH), 5.64 (s, 1H, H-2), 4.37 (d, J 15.1, 1H, Hb); 3.99 (d, J $\left.15.1,1 \mathrm{H}, \mathrm{H}_{\mathrm{a}}\right), 3.91\left(\mathrm{~s}, 3 \mathrm{H}, \mathrm{OCH}_{3}\right), 2.58\left(\mathrm{~s}, 3 \mathrm{H}, \mathrm{SCH}_{3}\right) .{ }^{13} \mathrm{C} \mathrm{NMR}\left(100 \mathrm{MHz}, \mathrm{CDCl}_{3}\right) \delta 166.3$ $(\mathrm{C}=\mathrm{N}), 153.7(\mathrm{C}=\mathrm{O}), 139.1$ (C-7a), 130.1 (C-3a), 129.8 (C-6), 124.1 (C-4), 124.0 (C-5), 114.9 (C-7), $88.0(\mathrm{C}-2), 75.2\left(\mathrm{CH}_{2}\right), 73.4(\mathrm{C}-3), 53.2\left(\mathrm{OCH}_{3}\right), 15.1\left(\mathrm{SCH}_{3}\right)$. NOESY correlations (400 $\left.\mathrm{MHz}, \mathrm{CDCl}_{3}\right): \mathrm{H}_{\mathrm{a}} / \mathrm{H}_{\mathrm{b}}, \mathrm{H}_{\mathrm{b}} / \mathrm{H}-2, \mathrm{H}-4 / \mathrm{H}-5, \mathrm{H}-5 / \mathrm{H}-6$.

trans-( \pm$)$ - and cis-( \pm$)-1-B o c-s p i r o b r a s s i n o l ~[t r a n s-( \pm)-29 a$ and cis- $( \pm)-29 b]$. To a stirred solution of 1-Boc-brassinin $(\mathbf{1 2} ; 0.027 \mathrm{~g}, 0.08 \mathrm{mmol})$ in a mixture of $\mathrm{CH}_{2} \mathrm{Cl}_{2} /$ water $(0.9 \mathrm{~mL} / 0.1$ $\mathrm{mL})$ at $\mathrm{rt}$ was added freshly prepared solution of $\mathrm{Br}_{2}(0.20 \mathrm{~mL}, 0.088 \mathrm{mmol})$. The stock solution was obtained by dissolving of $0.04 \mathrm{~mL}$ of bromine in $1.76 \mathrm{~mL}$ of anhydrous $\mathrm{CH}_{2} \mathrm{Cl}_{2}$. The reaction mixture was stirred for $15 \mathrm{~min}$, then $\mathrm{Et}_{3} \mathrm{~N}(0.017 \mathrm{~g}, 0.024 \mathrm{~mL}, 0.18 \mathrm{mmol})$ was added. Stirring was continued for $5 \mathrm{~min}$ and the reaction mixture was diluted with $\mathrm{CH}_{2} \mathrm{Cl}_{2}(5 \mathrm{~mL})$ and washed with brine $(2 \times 5 \mathrm{~mL})$. The organic layer was dried over anhydrous $\mathrm{Na}_{2} \mathrm{SO}_{4}$. The residue obtained after evaporation of the solvent was subjected to chromatography on $8 \mathrm{~g}$ silica gel ( $n$ hexane/Me $\left.{ }_{2} \mathrm{CO} 3: 1\right)$ and diastereoisomers trans-( \pm -29a and cis-( \pm -29b were separated.

trans-( \pm )-1-Boc-spirobrassinol [trans-( \pm )-29a]. Yield: $0.012 \mathrm{~g}(42 \%)$, white solid, $R_{f} 0.35$ ( $n$ hexane/ $\left.\mathrm{Me}_{2} \mathrm{CO} 3: 1\right)$, mp $73-75{ }^{\circ} \mathrm{C}\left(\mathrm{CHCl}_{3} /\right.$ light petroleum $)$. The spectral data were fully identical with those of previously described product trans- $( \pm)-29 a^{12}$

cis-( \pm$)-1-B o c-s p i r o b r a s s i n o l ~[c i s-( \pm)-29 b]$. Yield: $0.003 \mathrm{~g}(11 \%)$, colourless plates, $R_{f} 0.67$ (n-hexane/Me $\left.\mathrm{Me}_{2} \mathrm{CO} 3: 1\right)$, mp 126-128 ${ }^{\circ} \mathrm{C}\left(\mathrm{CH}_{2} \mathrm{Cl}_{2} /\right.$ light petroleum). The spectral data were fully identical with those of previously described product $c i s-( \pm)-29 b^{12}$ 
General procedure for the spirocyclization of 1-acyl derivatives of brassinin 23-25 with bromine in the presence of methanol. To a stirred solution of 1-acyl derivatives of brassinin 23-25 $(0.5 \mathrm{mmol})$ in a mixture of anhydrous $\mathrm{CH}_{2} \mathrm{Cl}_{2} / \mathrm{MeOH}(3.6 \mathrm{~mL} / 0.4 \mathrm{~mL})$ at rt was added freshly prepared solution of $\mathrm{Br}_{2}(1.26 \mathrm{~mL}, 0.55 \mathrm{mmol})$. The stock solution was obtained by dissolving of $0.04 \mathrm{~mL}$ of bromine in $1.76 \mathrm{~mL}$ of anhydrous $\mathrm{CH}_{2} \mathrm{Cl}_{2}$. The reaction mixture was stirred for $15 \mathrm{~min}$, then $\mathrm{Et} 3 \mathrm{~N}(0.111 \mathrm{~g}, 0.15 \mathrm{~mL}, 1.1 \mathrm{mmol})$ was added. Stirring was continued for $5 \mathrm{~min}$ and the reaction mixture was diluted with $\mathrm{CH}_{2} \mathrm{Cl}_{2}(25 \mathrm{~mL})$ and washed with brine (2 $\times 25 \mathrm{~mL}$ ). The organic layer was dried over anhydrous $\mathrm{Na}_{2} \mathrm{SO}_{4}$ and the residue obtained after evaporation of the solvent subjected to chromatography.

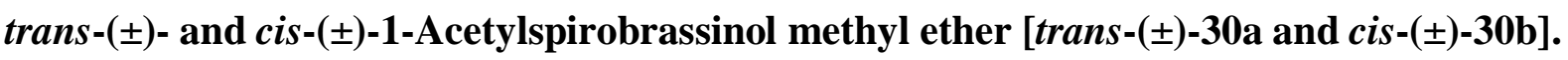
Following the general procedure, products trans $-( \pm)-30 \mathbf{a}$ and cis- $( \pm)-30 \mathbf{b}$ were obtained using $0.139 \mathrm{~g}(0.5 \mathrm{mmol})$ of 1-acetylbrassinin (23) and separated on silica gel ( $25 \mathrm{~g}, n$-hexane/EtOAc 1:1). Both diastereoisomers trans-( \pm$)-\mathbf{3 0 a}$ and cis- $( \pm)-30 \mathbf{b}$ were crystallized from $\mathrm{Et}_{2} \mathrm{O} / n$ hexane.

trans-( \pm )-1-Acetylspirobrassinol methyl ether [trans-( \pm )-30a]. Yield: $0.064 \mathrm{~g}(42 \%)$, white crystals, $R_{f} 0.63$ (n-hexane/EtOAc 1:1), mp 89-91 ${ }^{\circ} \mathrm{C}$ (Et ${ }_{2} \mathrm{O} / n$-hexane). Anal. Calcd for $\mathrm{C}_{14} \mathrm{H}_{16} \mathrm{~N}_{2} \mathrm{O}_{2} \mathrm{~S}_{2}$ requires: C, 54.52; H, 5.23; N, 9.08. Found: C, 54.76; H, 5.08; N, 9.32. MS (EI), $m / z$ (\%): $309[\mathrm{M}+\mathrm{H}]^{+}(68), 265(56), 43\left[\mathrm{CH}_{3} \mathrm{CO}\right]^{+}(100)$. IR $\left(\mathrm{CHCl}_{3}\right) v_{\max }: 2993,1653(\mathrm{C}=\mathrm{O})$, $1553(\mathrm{C}=\mathrm{N}), 1467,1373,1080 \mathrm{~cm}^{-1} .{ }^{1} \mathrm{H} \mathrm{NMR}\left(400 \mathrm{MHz}, \mathrm{CDCl}_{3}\right) \delta 8.12(\mathrm{~d}, J 6.3,1 \mathrm{H}, \mathrm{H}-7)$, 7.38 (d, J 7.3, 1H, H-4), 7.33-7.29 (m, 1H, H-6), 7.14-7.11 (m, 1H, H-5), 5.41 (s, 1H, H-2), $4.83\left(\mathrm{~d}, J 15.7,1 \mathrm{H}, \mathrm{H}_{\mathrm{b}}\right), 4.35\left(\mathrm{~d}, J 15.7,1 \mathrm{H}, \mathrm{H}_{\mathrm{a}}\right), 3.34\left(\mathrm{~s}, 3 \mathrm{H}, \mathrm{CH}_{3} \mathrm{O}\right), 2.58\left(\mathrm{~s}, 3 \mathrm{H}, \mathrm{SCH}_{3}\right), 2.37$ $\left(\mathrm{s}, 3 \mathrm{H}, \mathrm{CH}_{3} \mathrm{C}=\mathrm{O}\right) .{ }^{13} \mathrm{C} \mathrm{NMR}\left(100 \mathrm{MHz}, \mathrm{CDCl}_{3}\right) \delta 169.5(\mathrm{C}=\mathrm{O}), 164.8(\mathrm{C}=\mathrm{N}), 142.1(\mathrm{C}-7 \mathrm{a})$, 130.1 (C-6), 128.9 (C-3a), 124.6 (C-5), 123.4 (C-4), 117.1 (C-7), 100.2 (C-2), 71.1 (C-3), 66.6 $\left(\mathrm{CH}_{2}\right), 55.6\left(\mathrm{OCH}_{3}\right), 23.4\left(\mathrm{CH}_{3} \mathrm{C}=\mathrm{O}\right), 15.2\left(\mathrm{SCH}_{3}\right)$. NOESY correlations (400 MHz, $\left.\mathrm{CDCl}_{3}\right)$ : $\mathrm{H}_{\mathrm{a}} / \mathrm{H}_{\mathrm{b}}, \mathrm{H}_{\mathrm{a}} / \mathrm{H}-4, \mathrm{H}-6 / \mathrm{H}-7, \mathrm{H}-4 / \mathrm{H}-5$.

cis-( \pm -1-Acetylspirobrasinol methyl ether [( \pm -30b]. Yield: $0.038 \mathrm{~g}(25 \%)$, white crystals, $R_{f} 0.46$ (n-hexane/EtOAc 1:1), mp 91-93 ${ }^{\circ} \mathrm{C}$ (Et $2 \mathrm{O} / n$-hexane). Anal. Calcd for $\mathrm{C}_{14} \mathrm{H}_{16} \mathrm{~N}_{2} \mathrm{O}_{2} \mathrm{~S}_{2}$ requires: C, 54.52; H, 5.23; N, 9.08. Found: C, 54.86; H, 4.97; N, 9.31. MS of compound cis$( \pm)-30 b$ was fully identical with MS of trans-( \pm )-30a diastereoisomer. IR $\left(\mathrm{CHCl}_{3}\right) v_{\max }: 3007$, $1653(\mathrm{C}=\mathrm{O}), 1546(\mathrm{C}=\mathrm{N}), 1467,1373,1087 \mathrm{~cm}^{-1} .{ }^{1} \mathrm{H}$ NMR $\left(400 \mathrm{MHz}, \mathrm{CDCl}_{3}\right) \delta 8.15(\mathrm{~d}, J$ 7.1, 1H, H-7), 7.42 (d, J 7.5, 1H, H-4), 7.32-7.27 (m, 1H, H-6), 7.14-7.10 (m, 1H, H-5), 5.20 (s, 1H, H-2), $4.34\left(\mathrm{~d}, J 15.2,1 \mathrm{H}, \mathrm{H}_{\mathrm{b}}\right), 3.93\left(\mathrm{~d}, J 15.2,1 \mathrm{H}, \mathrm{H}_{\mathrm{a}}\right), 3.36\left(\mathrm{~s}, 3 \mathrm{H}, \mathrm{CH}_{3} \mathrm{O}\right), 2.59(\mathrm{~s}, 3 \mathrm{H}$, $\left.\mathrm{SCH}_{3}\right), 2.37\left(\mathrm{~s}, 3 \mathrm{H}, \mathrm{CH}_{3} \mathrm{C}=\mathrm{O}\right) .{ }^{13} \mathrm{C} \mathrm{NMR}\left(100 \mathrm{MHz}, \mathrm{CDCl}_{3}\right) \delta 169.7(\mathrm{C}=\mathrm{O}), 167.1(\mathrm{C}=\mathrm{N})$, 140.9 (C-7a), 130.2 (C-3a), 129.8 (C-6), 124.7 (C-5), 123.3 (C-4), 116.7 (C-7), 95.3 (C-2), 75.7 $\left(\mathrm{CH}_{2}\right), 72.9(\mathrm{C}-3), 55.3\left(\mathrm{OCH}_{3}\right), 23.5\left(\mathrm{CH}_{3} \mathrm{C}=\mathrm{O}\right), 15.1\left(\mathrm{SCH}_{3}\right)$. NOESY correlations (400 $\left.\mathrm{MHz}, \mathrm{CDCl}_{3}\right): \mathrm{H}_{\mathrm{a}} / \mathrm{H}_{\mathrm{b}}, \mathrm{H}_{\mathrm{b}} / \mathrm{H}-2$, H-6/H-7, H-4/H-5.

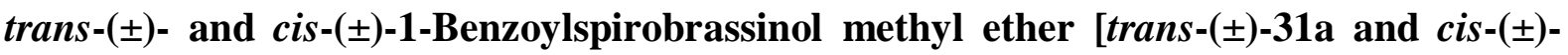
31b]. Following the general procedure, products trans-( \pm )-31a and cis- $( \pm)-31 \mathbf{b}$ were obtained using $0.170 \mathrm{~g}(0.5 \mathrm{mmol})$ of 1-benzoylbrassinin (24) and separated on silica gel (40 $\mathrm{g}, n$ hexane/Et $\left.{ }_{2} \mathrm{O} 1: 1\right)$. Both diastereoisomers trans- $( \pm)-31 \mathbf{a}$ and cis- $( \pm)-31 \mathbf{b}$ were crystallized from $\mathrm{Me}_{2} \mathrm{CO} / n$-hexane.

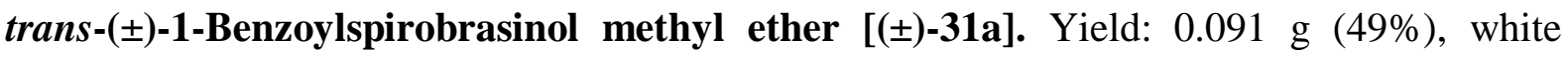
crystals, $R_{f} 0.38$ ( $n$-hexane/Et $\left.2 \mathrm{O} 1: 1\right)$, mp 112-115 ${ }^{\circ} \mathrm{C}\left(\mathrm{Me}_{2} \mathrm{CO} / n\right.$-hexane). Anal. Calcd for 
$\mathrm{C}_{19} \mathrm{H}_{18} \mathrm{~N}_{2} \mathrm{O}_{2} \mathrm{~S}_{2}$ requires: $\mathrm{C}, 61.60 ; \mathrm{H}, 4.90 ; \mathrm{N}, 7.56$. Found: $\mathrm{C}, 61.91 ; \mathrm{H}, 4.64 ; \mathrm{N}, 7.82$. MS (EI), $m / z(\%): 371[\mathrm{M}+\mathrm{H}]^{+}(5), 370[\mathrm{M}]^{+}(39), 265(72), 105\left[\mathrm{C}_{6} \mathrm{H}_{5} \mathrm{C}=\mathrm{O}\right]^{+}(98), 77\left[\mathrm{C}_{6} \mathrm{H}_{5}\right]^{+}$(100). IR $\left(\mathrm{CHCl}_{3}\right) v_{\max }: 3006,1675(\mathrm{C}=\mathrm{O}), 1560(\mathrm{C}=\mathrm{N}), 1469,1372,1092 \mathrm{~cm}^{-1} .{ }^{1} \mathrm{H}$ NMR $(400 \mathrm{MHz}$, $\left.\mathrm{CDCl}_{3}\right) \delta 7.57$ (d, J 6.6, 2H, H-2', H-6'), 7.52-7.44 (m, 4H, H-4', H-3’' H-5', H-7), 7.39 (d, J 7.4, 1H, H-4), 7.26-7.10 (m, 2H, H-6, H-5), 5.52 (s, 1H, H-2), 4.79 (d, J 15.7, 1H, Hb), 4.36 (d, $J$ 15.7, $\left.1 \mathrm{H}, \mathrm{H}_{\mathrm{a}}\right), 3.21\left(\mathrm{~s}, 3 \mathrm{H}, \mathrm{OCH}_{3}\right), 2.56\left(\mathrm{~s}, 3 \mathrm{H}, \mathrm{SCH}_{3}\right) .{ }^{13} \mathrm{C} \mathrm{NMR}\left(100 \mathrm{MHz}, \mathrm{CDCl}_{3}\right) \delta 169.7$ $(\mathrm{C}=\mathrm{O}), 164.5(\mathrm{C}=\mathrm{N}), 141.6$ (C-7a), $135.5\left(\mathrm{C}-1^{\prime}\right), 130.8$ (C-3a, C-4'), 129.6 (C-6), 128.6 (C-3', C-5'), 127.6 (C-2’, C-6’), 124.8 (C-5), 123.5 (C-4), 117.4 (C-7), 99.9 (C-2), 70.9 (C-3), 66.2 $\left(\mathrm{CH}_{2}\right), 57.2\left(\mathrm{OCH}_{3}\right), 15.2\left(\mathrm{SCH}_{3}\right)$. NOESY correlations (400 MHz, $\left.\mathrm{CDCl}_{3}\right): \mathrm{H}_{\mathrm{a}} / \mathrm{H}-4, \mathrm{H}_{\mathrm{a}} / \mathrm{H}_{\mathrm{b}}$.

cis-( \pm -1-Benzoylspirobrasinol methyl ether [( \pm -31b]. Yield: $0.031 \mathrm{~g}(17 \%)$, white crystals, $R_{f} 0.27$ (n-hexane/Et ${ }_{2} \mathrm{O}$ 1:1), mp 113-116 ${ }^{\circ} \mathrm{C}$ ( $\mathrm{Me}_{2} \mathrm{CO} / n$-hexane). Anal. Calcd for $\mathrm{C}_{19} \mathrm{H}_{18} \mathrm{~N}_{2} \mathrm{O}_{2} \mathrm{~S}_{2}$ requires: $\mathrm{C}, 61.60 ; \mathrm{H}, 4.90 ; \mathrm{N}, 7.56$. Found: $\mathrm{C}, 61.33 ; \mathrm{H}, 5.19 ; \mathrm{N}, 7.30$. MS of

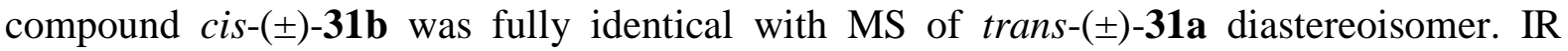
$\left(\mathrm{CHCl}_{3}\right) v_{\max }:$ 3006, $1668(\mathrm{C}=\mathrm{O}), 1560(\mathrm{C}=\mathrm{N}), 1461,1370,1098 \mathrm{~cm}^{-1} .{ }^{1} \mathrm{H}$ NMR $(400 \mathrm{MHz}$, $\left.\mathrm{CDCl}_{3}\right) \delta 7.57$-7.38 (m, 7H, H-2', H-6', H-4', H-3', H-5', H-4, H-7), 7.22-7.18 (m, 1H, H-6), 7.14-7.10 (m, 1H, H-5), 5.22 (s, 1H, H-2), 4.42 (d, J 15.1, 1H, Hb), 3.98 (d, J 15.1, 1H, $\mathrm{H}_{\mathrm{a}}$ ), $3.25\left(\mathrm{~s}, 3 \mathrm{H}, \mathrm{OCH}_{3}\right), 2.57\left(\mathrm{~s}, 3 \mathrm{H}, \mathrm{SCH}_{3}\right) .{ }^{13} \mathrm{C} \mathrm{NMR}\left(100 \mathrm{MHz}, \mathrm{CDCl}_{3}\right) \delta 170.2(\mathrm{C}=\mathrm{O}), 167.2$ $(\mathrm{C}=\mathrm{N}), 140.6$ (C-7a), 135.9 (C-1'), 132.4 (C-3a), 131.1 (C-4'), 129.4 (C-6), 128.9 (C-3', C5'), 127.7 (C-2', C-6’), 125.2 (C-5), 124.0 (C-4), 117.4 (C-7), 96.9 (C-2), $74.8\left(\mathrm{CH}_{2}\right), 73.6$ (C3), $57.9\left(\mathrm{OCH}_{3}\right), 15.3\left(\mathrm{SCH}_{3}\right)$. NOESY correlations (400 MHz, $\left.\mathrm{CDCl}_{3}\right): \mathrm{H}_{\mathrm{a}} / \mathrm{H}_{\mathrm{b}}, \mathrm{Hb} / \mathrm{H}-2$.

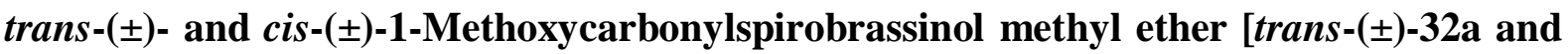
cis-( \pm$)-32 \mathbf{b}]$. Following the general procedure, products trans-( \pm$)-32 \mathbf{a}$ and cis- $( \pm)-32 \mathbf{b}$ were obtained using $0.147 \mathrm{~g}(0.5 \mathrm{mmol})$ of 1-(methoxycarbonyl)brassinin (25) and separated on silica gel (30 g, $n$-hexane/EtOAc 2:1). Diastereoisomer trans-( \pm )-32a was crystallized from $\mathrm{Et}_{2} \mathrm{O} / n$-hexane. Diastereoisomer cis- $( \pm)-32 \mathbf{b}$ was isolated as a colourless oil.

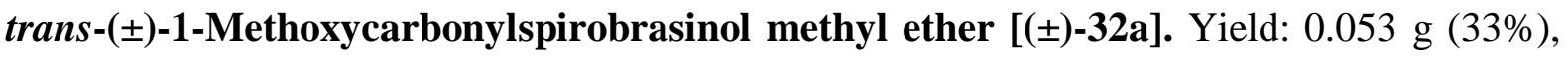
white crystals, $R_{f} 0.48$ ( $n$-hexane/EtOAc $\left.2: 1\right), \mathrm{mp} 125-128{ }^{\circ} \mathrm{C}\left(\mathrm{Et}_{2} \mathrm{O} / n\right.$-hexane). Anal. Calcd for $\mathrm{C}_{14} \mathrm{H}_{16} \mathrm{~N}_{2} \mathrm{O}_{3} \mathrm{~S}_{2}$ requires: $\mathrm{C}, 51.83 ; \mathrm{H}, 4.97 ; \mathrm{N}, 8.63$. Found: $\mathrm{C}, 52.09 ; \mathrm{H}, 4.73 ; \mathrm{N}, 8.82$. MS (EI), $m / z(\%): 325[\mathrm{M}+\mathrm{H}]^{+}(15), 324[\mathrm{M}]^{+}(96), 245(100), 87(71), 72(57), 59{\left[\mathrm{CH}_{3} \mathrm{OCO}\right]^{+}}^{+}$ (98). IR ( $\left.\mathrm{CHCl}_{3}\right) v_{\max }: 1702(\mathrm{C}=\mathrm{O}), 1553(\mathrm{C}=\mathrm{N}), 1476,1436,1372,1066 \mathrm{~cm}^{-1} .{ }^{1} \mathrm{H}$ NMR (400 $\left.\mathrm{MHz} \mathrm{CDCl}_{3}\right) \delta 7.76$ (bs, 1H, H-7), 7.35 (d, J 7.5, 1H, H-4), 7.31-7.26 (m, 1H, H-6), 7.09-7.05 (m, 1H, H-5), 5.56 (s, 1H, H-2), $4.86\left(\mathrm{~d}, J 15.6,1 \mathrm{H}, \mathrm{H}_{\mathrm{b}}\right), 4.33\left(\mathrm{~d}, J 15.6,1 \mathrm{H}, \mathrm{H}_{\mathrm{a}}\right), 3.90(\mathrm{~s}, 3 \mathrm{H}$, $\left.\mathrm{COOCH}_{3}\right), 3.50\left(\mathrm{~s}, 3 \mathrm{H}, \mathrm{OCH}_{3}\right), 2.57\left(\mathrm{~s}, 3 \mathrm{H}, \mathrm{SCH}_{3}\right) .{ }^{13} \mathrm{C} \mathrm{NMR}\left(100 \mathrm{MHz}, \mathrm{CDCl}_{3}\right) \delta 164.6$ $(\mathrm{C}=\mathrm{N}), 153.8(\mathrm{C}=\mathrm{O}), 140.8$ (C-7a), 129.8 (C-6, C-3a), 124.0 (C-5), 123.5 (C-4), 115.9 (C-7), $98.8(\mathrm{C}-2), 70.7(\mathrm{C}-3), 66.3\left(\mathrm{CH}_{2}\right), 57.8\left(\mathrm{OCH}_{3}\right), 53.1\left(\mathrm{COOCH}_{3}\right), 15.1\left(\mathrm{SCH}_{3}\right)$. NOESY correlations (400 MHz, CDCl 3 ): $\mathrm{H}_{\mathrm{a}} / \mathrm{H}-4, \mathrm{H}-2 / \mathrm{OCH}_{3}, \mathrm{H}-4 / \mathrm{H}-5, \mathrm{H}-6 / \mathrm{H}-7$.

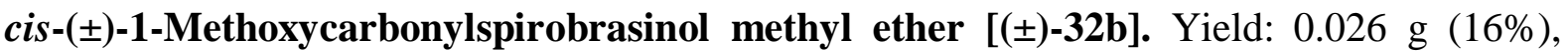
colourless oil, $R_{f} 0.38$ ( $n$-hexane/EtOAc 2:1). Anal. Calcd for $\mathrm{C}_{14} \mathrm{H}_{16} \mathrm{~N}_{2} \mathrm{O}_{3} \mathrm{~S}_{2}$ requires: C, 51.83 ; $\mathrm{H}, 4.97$; N, 8.63. Found: C, 52.11; H, 4.69; N, 8.47. MS of compound cis-( \pm )-32b was fully identical with MS of trans-( \pm -32a diastereoisomer. IR $\left(\mathrm{CHCl}_{3}\right) v_{\max }: 1699(\mathrm{C}=\mathrm{O}), 1560$ $(\mathrm{C}=\mathrm{N}), 1460,1433,1368,1266,1085 \mathrm{~cm}^{-1} .{ }^{1} \mathrm{H} \mathrm{NMR}\left(400 \mathrm{MHz}, \mathrm{CDCl}_{3}\right) \delta 7.81$ (bs, $\left.1 \mathrm{H}, \mathrm{H}-7\right)$, 7.36 (d, J 7.5, 1H, H-4), 7.30-7.26 (m, 1H, H-6), 7.08-7.05 (m, 1H, H-5), 5.29 (s, 1H, H-2), $4.34\left(\mathrm{~d}, J 15.1,1 \mathrm{H}, \mathrm{H}_{\mathrm{b}}\right), 3.91\left(\mathrm{~s}, 3 \mathrm{H}, \mathrm{COOCH}_{3}\right), 3.90\left(\mathrm{~d}, J 15.1,1 \mathrm{H}, \mathrm{H}_{\mathrm{a}}\right), 3.53\left(\mathrm{~s}, 3 \mathrm{H}, \mathrm{OCH}_{3}\right)$, 
$2.58\left(\mathrm{~s}, 3 \mathrm{H}, \mathrm{SCH}_{3}\right) .{ }^{13} \mathrm{C} \mathrm{NMR}\left(100 \mathrm{MHz}, \mathrm{CDCl}_{3}\right) \delta 166.8(\mathrm{C}=\mathrm{N}), 153.3(\mathrm{C}=\mathrm{O}), 139.4(\mathrm{C}-7 \mathrm{a})$, 131.7 (C-3a), 129.5 (C-6), 124.0 (C-5), 123.7 (C-4), 115.5 (C-7), 95.6 (C-2), 74.8 ( $\left.\mathrm{CH}_{2}\right), 73.2$ $(\mathrm{C}-3), 58.2\left(\mathrm{OCH}_{3}\right), 53.1\left(\mathrm{COOCH}_{3}\right), 15.1\left(\mathrm{SCH}_{3}\right)$. NOESY correlations $\left(400 \mathrm{MHz}, \mathrm{CDCl}_{3}\right)$ : $\mathrm{H}_{\mathrm{a}} / \mathrm{H}_{\mathrm{b}}, \mathrm{H}_{\mathrm{b}} / \mathrm{H}-2, \mathrm{H}-2 / \mathrm{OCH}_{3}, \mathrm{H}-4 / \mathrm{H}-5, \mathrm{H}-5 / \mathrm{H}-6, \mathrm{H}-6 / \mathrm{H}-7$.

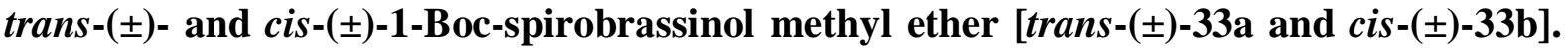
Method A: To a stirred solution of 1-Boc-brassinin $(12 ; 0.027 \mathrm{~g}, 0.08 \mathrm{mmol})$ in a mixture of $\mathrm{CH}_{2} \mathrm{Cl}_{2} / \mathrm{MeOH}(0.9 \mathrm{~mL} / 0.1 \mathrm{~mL})$ at $\mathrm{rt}$ was added freshly prepared solution of $\mathrm{Br}_{2}(0.20 \mathrm{~mL}$, $0.088 \mathrm{mmol}$ ). The stock solution was obtained by dissolving of $0.04 \mathrm{~mL}$ of bromine in 1.76 $\mathrm{mL}$ of anhydrous $\mathrm{CH}_{2} \mathrm{Cl}_{2}$. The reaction mixture was stirred for $15 \mathrm{~min}$, then $\mathrm{Et}_{3} \mathrm{~N}(0.017 \mathrm{~g}$, $0.024 \mathrm{~mL}, 0.18 \mathrm{mmol}$ ) was added. Stirring was continued for $5 \mathrm{~min}$ and the reaction mixture was diluted with $\mathrm{CH}_{2} \mathrm{Cl}_{2}(5 \mathrm{~mL})$ and washed with brine $(2 \times 5 \mathrm{~mL})$. The organic layer was dried over anhydrous $\mathrm{Na}_{2} \mathrm{SO}_{4}$. The residue obtained after evaporation of the solvent was subjected to chromatography on $5 \mathrm{~g}$ silica gel (petroleum ether/EtOAc 5:1), affording mixture of products trans-( \pm$)-33 \mathbf{a}$ : cis- $( \pm)-33 \mathbf{b}$ in a 71:29 ratio. Subsequent chromatography of the mixture of diastereoisomers $( \pm)-33 \mathbf{a}$ and $( \pm)-33 \mathbf{b}$ on $5 \mathrm{~g}$ of silica gel $\left(\mathrm{CH}_{2} \mathrm{Cl}_{2}\right)$ gave $( \pm)-33 \mathbf{a}$ $(0.013 \mathrm{~g}, 45 \%)$ and $( \pm)-33 b(0.006 \mathrm{~g}, 20 \%)$.

Method B: To a stirred solution of 1-Boc-brassinin $(12 ; 0.150 \mathrm{~g}, 0.446 \mathrm{mmol})$ in a mixture of 1,4-dioxane/MeOH (5.4 mL/0.6 mL) at $\mathrm{rt}$ was added freshly prepared solution of DDB (2.96 $\mathrm{mL}, 0.491 \mathrm{mmol}$ ). The stock solution was obtained by dissolving of $0.05 \mathrm{~mL}$ of bromine in 6.0 $\mathrm{mL}$ of 1,4-dioxane. The reaction mixture was stirred for $15 \mathrm{~min}$, then $\mathrm{Et}_{3} \mathrm{~N}(0.99 \mathrm{~g}, 0.137 \mathrm{~mL}$, $0.971 \mathrm{mmol}$ ) was added. Stirring was continued for $5 \mathrm{~min}$ and the mixture poured mixture into water $(90 \mathrm{~mL})$, the product extracted with EtOAc $(2 \times 30 \mathrm{~mL})$, the extract washed with brine $(2 \times 30 \mathrm{~mL})$. The organic layer was dried over anhydrous $\mathrm{Na}_{2} \mathrm{SO}_{4}$ and the residue obtained after evaporation of the solvent subjected to chromatography on $15 \mathrm{~g}$ of silica gel (petroleum ether/EtOAc 5:1), affording mixture of products trans-( \pm -33a : cis-( \pm )-33b in a 71:29 ratio. Subsequent chromatography of the mixture of diastereoisomers $( \pm)-33 \mathbf{a}$ and $( \pm)-33 \mathbf{b}$ on $15 \mathrm{~g}$ of silica gel $\left(\mathrm{CH}_{2} \mathrm{Cl}_{2}\right)$ gave ( \pm )-33a $(0.068 \mathrm{~g}, 42 \%)$ and $( \pm)-33 \mathbf{b}(0.014 \mathrm{~g}, 9 \%)$.

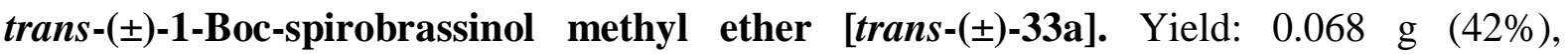
colourless solid, $R_{f} 0.12\left(\mathrm{CH}_{2} \mathrm{Cl}_{2}\right), \mathrm{mp} 68-70{ }^{\circ} \mathrm{C}$. The spectral data were fully identical with those of previously described product trans-( \pm -33a. ${ }^{31}$

cis-( \pm$)$-1-Boc-spirobrassinol methyl ether [cis-( \pm -33b]. Yield: $0.014 \mathrm{~g}(9 \%)$, colourless oil, $R_{f} 0.19\left(\mathrm{CH}_{2} \mathrm{Cl}_{2}\right)$. The spectral data were fully identical with those of previously described product cis- $( \pm)-33 \mathbf{b} .^{31}$

Spirocyclization of brassinin (5b) or 1-methylbrassinin (37) with DDB (1.1 eq.) in the presence of methanol. To a stirred solution of brassinin $(5 \mathbf{b} ; 0.035 \mathrm{~g}, 0.15 \mathrm{mmol})$ or 1 methylbrassinin $(37 ; 0.038 \mathrm{~g}, 0.15 \mathrm{mmol})$ in a mixture of 1,4-dioxane/MeOH $(1.8 \mathrm{~mL} / 0.2 \mathrm{~mL})$ at $\mathrm{rt}$ was added freshly prepared solution of DDB $(0.38 \mathrm{~mL}, 0.165 \mathrm{mmol})$. The stock solution was obtained by dissolving of $0.04 \mathrm{~mL}$ of bromine in $1.76 \mathrm{~mL}$ of anhydrous of 1,4-dioxane. The reaction mixture was stirred for $15 \mathrm{~min}$, then $\mathrm{Et}_{3} \mathrm{~N}(0.033 \mathrm{~g}, 0.046 \mathrm{~mL}, 0.33 \mathrm{mmol})$ was added. Stirring was continued for $5 \mathrm{~min}$ and the mixture poured mixture into water $(10 \mathrm{~mL})$, the product extracted with $\mathrm{CH}_{2} \mathrm{Cl}_{2}(2 \times 10 \mathrm{~mL})$, the extract washed with brine $(2 \times 10 \mathrm{~mL})$. 
The organic layer was dried over anhydrous $\mathrm{Na}_{2} \mathrm{SO}_{4}$ and the residue obtained after evaporation of the solvent subjected to chromatography on $5 \mathrm{~g}$ of silica gel ( $n$-hexane/EtOc 2:1).

Spirobrassinin [( \pm )-1]. Yield: $0.018 \mathrm{~g}(47 \%)$, colourless crystals, $R_{f} 0.24$ ( $n$-hexane/ EtOAc 2:1), mp 159-160 ${ }^{\circ} \mathrm{C}\left(\mathrm{Me}_{2} \mathrm{CO} / n\right.$-hexane). The spectral data were fully identical with those of natural product $[(-)-\mathbf{1}] .^{5}$

1-Methylspirobrassinin [( \pm )-44]. Yield: $0.022 \mathrm{~g}$ (55\%), white solid, $R_{f} 0.25$ ( $n$-hexane/EtOAc $2: 1)$. The spectral data were fully identical with those of previously described product $( \pm)-44 .{ }^{36}$

Spirocyclization of brassinin (5b) with DDB (4 eq.) in the presence of methanol. To a stirred solution of brassinin $(\mathbf{5 b} ; 0.035 \mathrm{~g}, 0.15 \mathrm{mmol})$ in a mixture of 1,4-dioxane/MeOH (1.8 $\mathrm{mL} / 0.2 \mathrm{~mL})$ at $\mathrm{rt}$ was added freshly prepared solution of DDB $(1.4 \mathrm{~mL}, 0.6 \mathrm{mmol})$. The stock solution was obtained by dissolving of $0.04 \mathrm{~mL}$ of bromine in $1.76 \mathrm{~mL}$ of anhydrous of 1,4dioxane. The reaction mixture was stirred for $15 \mathrm{~min}$, then $\mathrm{Et}_{3} \mathrm{~N}(0.121 \mathrm{~g}, 0.167 \mathrm{~mL}, 1.2 \mathrm{mmol})$ was added. Stirring was continued for $5 \mathrm{~min}$ and the mixture poured mixture into water (10 $\mathrm{mL})$, the product extracted with $\mathrm{CH}_{2} \mathrm{Cl}_{2}(2 \times 10 \mathrm{~mL})$, the extract washed with brine $(2 \times 10$ $\mathrm{mL}$ ). The organic layer was dried over anhydrous $\mathrm{Na}_{2} \mathrm{SO}_{4}$ and the residue obtained after evaporation of the solvent subjected to chromatography on $5 \mathrm{~g}$ of silica gel ( $n$-hexane/EtOAc 2:1).

5-Bromospirobrassinin [( \pm )-43]. Yield: $0.024 \mathrm{~g}(49 \%)$, pale yellow oil, $R_{f} 0.22$ (nhexane/EtOAc 2:1). The spectral data were fully identical with those of previously described product $( \pm)-\mathbf{4 3} .^{37}$

Spirocyclization of 5-bromobrassinin (5c) with DDB (4 eq.) in the presence of methanol. To a stirred solution of brassinin $(\mathbf{5 c} ; 0.047 \mathrm{~g}, 0.15 \mathrm{mmol})$ in a mixture of 1,4-dioxane/MeOH $(1.8 \mathrm{~mL} / 0.2 \mathrm{~mL})$ at $\mathrm{rt}$ was added freshly prepared solution of DDB $(1.4 \mathrm{~mL}, 0.6 \mathrm{mmol})$. The stock solution was obtained by dissolving of $0.04 \mathrm{~mL}$ of bromine in $1.76 \mathrm{~mL}$ of anhydrous of 1,4-dioxane. The reaction mixture was stirred for $15 \mathrm{~min}$, then $\mathrm{Et}_{3} \mathrm{~N}(0.121 \mathrm{~g}, 0.167 \mathrm{~mL}, 1.2$ $\mathrm{mmol}$ ) was added. Stirring was continued for $5 \mathrm{~min}$ and the mixture poured mixture into water $(10 \mathrm{~mL})$, the product extracted with $\mathrm{CH}_{2} \mathrm{Cl}_{2}(2 \times 10 \mathrm{~mL})$, the extract washed with brine $(2 \times$ $10 \mathrm{~mL}$ ). The organic layer was dried over anhydrous $\mathrm{Na}_{2} \mathrm{SO}_{4}$ and the residue obtained after evaporation of the solvent subjected to chromatography on $5 \mathrm{~g}$ of silica gel ( $n$-hexane/EtOAc 2:1).

5-Bromospirobrassinin [( \pm )-43]. Yield: 0.031 g (64\%), pale yellow oil, $R_{f} 0.22$ (nhexane/EtOAc 2:1). The spectral data were fully identical with those of previously described product $( \pm)-\mathbf{4 3} .^{37}$

\section{Biological effects}

Cell lines. Jurkat (human T-cell acute lymphoblastic leukemia), HeLa (human cervical adenocarcinoma) and MCF-7 (human breast adenocarcinoma, estrogen receptor-positive) were obtained from the European Collection of Cell Cultures (United Kingdom), CCRF-CEM cell line (human T-cell acute lymphoblastic leukemia) from the German Collection of Microorganisms and Cell Cultures (Braunschweig, Germany). MDA-MB-231 (human breast 
adenocarcinoma, estrogen receptor-negative) and A-549 cell lines (human lung adenocarcinoma) were kindly provided by Dr. M. Hajdúch (Olomouc, Czech Republic). The cells were routinely maintained in RPMI 1640 medium with L-glutamine and HEPES (Jurkat, HeLa and CCR-CEM) or Dulbecco's modified Eagle's medium with Glutamax- I (MCF-7, MDA-MB-231 and A-549) supplemented with 10\% fetal calf serum, penicillin (100 IU x mL ${ }^{-1}$ ) and streptomycin (100 $\lg \times \mathrm{mL}^{-1}$ ) (all from Invitrogen, USA), in humidified air with $5 \% \mathrm{CO}_{2}$ at $37{ }^{\circ} \mathrm{C}$. Before each cytotoxicity assay, cell viability was determined by the trypan blue exclusion method and found to be greater than $95 \%$.

Cytotoxicity assay. The antiproliferative effects of compounds were studied using the colorimetric microculture assay with the MTT endpoint. ${ }^{38}$ Briefly, $5 \times 10^{3}$ cells were plated per well in 96-well polystyrene microplates (Sarstedt, Germany) in $100 \mu \mathrm{L}$ of the culture medium containing tested chemicals at final concentrations of $10^{-6}-10^{-4} \mathrm{~mol} \times \mathrm{L}^{-1}$. After $72 \mathrm{~h}$ incubation, $10 \mu \mathrm{L}$ of MTT ( $5 \mathrm{mg} \times \mathrm{mL}^{-1}$, Sigma-Aldrich) was added into each well. After an additional $4 \mathrm{~h}$ at $37^{\circ} \mathrm{C}$, during which insoluble formazan was produced, $100 \mu \mathrm{L}$ of $10 \%(\mathrm{~m} / \mathrm{m})$ sodium dodecylsulfate (SDS, Sigma-Aldrich) was added into each well and another $12 \mathrm{~h}$ were allowed for the dissolution of formazan. The absorbance was measured at $540 \mathrm{~nm}$ and $630 \mathrm{~nm}$ - reference wavelenght by the automated uQuant ${ }^{\mathrm{TM}}$ Universal Microplate Spectrophotometer (Biotek Instruments Inc., Winooski, VT USA). The blank corrected absorbance of the control wells was taken as $100 \%$ and the results were expressed as a percentage of the control.

\section{Acknowledgements}

We would like to thank the Slovak Grant Agency for Science (Grant Nos. 1/0954/12 and 1/0322/14) for financial support of this work.

\section{References}

1. Müller, K. O.; Börger, H. Arb. Biol. Reichsanst Land. Forstwirtsch. Berlin-Dahlem 1940, 23, 189.

2. Mansfield, J. W.; Bailey, J .A. in Phytoalexins, John Wiley, New York, 1982, 319-323.

3. Takasugi, M.; Katsui, N.; Shirata, A. J. Chem. Soc., Chem. Commun. 1986, 1077. https://doi.org/10.1039/c39860001077

4. $\quad$ Pedras, M. S. C.; Yaya, E. E.; Glawischnig, E. Nat. Prod. Rep. 2011, 28, 1381. https://doi.org/10.1039/c1np00020a

5. Takasugi, M.; Monde, K.; Katsui, N.; Shirata, A. Chem. Lett. 1987, 1631. https://doi.org/10.1246/cl.1987.1631

6. Gross, D.; Porzel, A.; Schmidt, J. Z. Naturforsch. C: Biosci. 1994, 49, 281.

7. Monde, K.; Takasugi, M.; Shirata, A. Phytochemistry 1995, 39, 581. https://doi.org/10.1016/0031-9422(95)00011-U 
8. Monde, K.; Osawa, S.; Harada, N.; Takasugi, M.; Suchý, M.; Kutschy, P.; Dzurilla, M.; Balentová, E. Chem. Lett. 2000, 886.

https://doi.org/10.1246/c1.2000.886

9. $\quad$ Suchý, M.; Kutschy, P.; Monde, K.; Goto, H.; Harada, N.; Takasugi, M.; Dzurilla, M.; Balentová, E. J. Org. Chem. 2001, 66, 3940.

https://doi.org/10.1021/jo0155052

10. Budovská, M.; Kutschy, P.; Kožár, T.; Gondová, T.; Petrovaj, J. Tetrahedron 2013, 69, 1092.

\section{https://doi.org/10.1016/j.tet.2012.11.067}

11. Monde, K.; Taniguchi, T.; Miura, N.; Kutschy, P.; Čurillová, Z.; Pilátová, M.; Mojžiš, J. Bioorg. Med. Chem. 2005, 13, 5206.

https://doi.org/10.1016/j.bmc.2005.06.001

12. Kutschy, P.; Suchý, M.; Monde, K.; Harada, N.; Marušková, R.; Čurillová, Z.; Dzurilla, M.; Miklošová, M.; Mezencev, R.; Mojžiš, J. Tetrahedron Lett. 2002, 43, 9489.

https://doi.org/10.1016/S0040-4039(02)02452-8

13. Storck, M.; Sacristan, M. D. Z. Naturforsch. 1995, 50c, 15.

14. Pedras, M. S. C.; Suchý, M.; Ahiahonu, P. W. K. Org. Biomol. Chem. 2006, 4, 691. https://doi.org/10.1039/b515331j

15. Mezencev, R.; Mojžiš, J.; Pilátová, M.; Kutschy, P. Neoplasma 2003, 50, 239.

16. Kutschy, P.; Mezencev, R. Indole Phytoalexins from Brassicaceae: Synthesis and Anticancer Activity. In: Targets in Heterocyclic Systems - Chemistry and Properties; Attanasi, O. A.; Spinelli, D., Eds.; Italian Society of Chemistry: Urbino, Italy, 2009; Vol. 12, pp 120-148. ISBN: 978-88-86208-56-7 ISSN 1724-9449.

17. Mehta, R. G.; Liu, J.; Constantinou, A.; Hawthorne, M.; Pezzuto, J. M.; Moon, R. C.; Moriarty, R. M. Anticancer Res. 1994, 14, 1209.

18. Tempete, C.; Devys, M.; Barbier, M. Z. Naturforsch. 1991, 46c, 706.

19. Mehta, R.G.; Liu, J.; Constantinou, A.; Thomas, C.F.; Hawthorne, M.; You, M.; Gerhuser, C.; Pezzuto, J.M.; Moon, R.C.; Moriarty, R.M. Carcinogenesis 1995, 16, 399.

https://doi.org/10.1093/carcin/16.2.399

20. Izutani, Y.; Yogosawa, S.; Sowa, Y.; Sakai, T. Int. J. Oncol. 2012, 40, 816.

21. Pilatova, M.; Sarissky, M.; Kutschy, P.; Mirossay, A.; Mezencev, R.; Curillova, Z.; Suchy, M.; Monde, K.; Mirossay, L.; Mojzis, J. Leuk. Res. 2005, 29, 415.

22. Mezencev, R.; Kutschy, P.; Salayova, A.; Curillova, Z.; Mojzis, J.; Pilatova, M.; McDonald, J. Chemotherapy 2008, 54, 372.

https://doi.org/10.1093/carcin/16.2.399

23. Kutschy, P.; Salayová, A.; Čurillová, Z.; Kožár, T.; Mezencev, R.; Mojžiš, J.; Pilátová, M.; Balentová, E.; Pazdera, P.; Sabol, M.; Zburová, M. Bioorg. Med. Chem. 2009, 17, 3698.

https://doi.org/10.1016/j.bmc.2009.03.064

24. Budovská, M.; Pilatová, M.; Varinská, L.; Mojžišs, J.; Mezencev, R. Bioorg. Med. Chem. 2013, 21, 6623.

https://doi.org/10.1016/j.bmc.2013.08.020 
25. Chripkova, M.; Drutovic, D.; Pilatova, M.; Mikes, J.; Budovska, M.; Vaskova, J.; Broggini, M.; Mirossay, L.; Mojzis, J. Toxicol. In Vitro 2014, 28, 907.

26. Kello, M.; Drutovic, D.; Chripková, M.; Pilatová, M.; Budovská, M.; Kuliková, L.; Urdzík, P.; Mojžiš, J. Molecules 2014, 19, 10877. https://doi.org/10.3390/molecules190810877

27. Gaspari, P.; Banerjee, T.; Malachowski, W. P.; Muller, A. J.; Prendergast, G. C.; DuHadaway, J.; Bennett, S.; Donovan, A. M. J. Med. Chem. 2006, 49, 684. https://doi.org/10.1021/jm0508888

28. Banerjee, T.; Duhadaway, J. B; Gaspari, P.; Sutanto-Ward, E.; Munn, D. H.; Mellor, A. L.; Malachowski, W. P.; Prendergast, G. C.; Muller, A. J. Oncogene 2008, 27, 2851. https://doi.org/10.1038/sj.onc.1210939

29. Mezencev, R.; Galizzi, M.; Kutschy, P.; Docampo, R. Exp. Parasitol. 2009, 122, 66. https://doi.org/10.1016/j.exppara.2009.01.013

30. Kristofikova, Z.; Gazova, Z.; Siposova, K.; Bartos, A.; Ricny, J.; Kotoucova, J.; Sirova, J.; Ripova, D. Neurochem. Res., 2014, 39, 1502.

https://doi.org/10.1007/s11064-014-1340-0

31. Budovská, M. RSC Adv. 2014, 4, 5575. https://doi.org/10.1039/c3ra46843g

32. Takasugi, M.; Monde, K.; Katsui, N.; Shirata, A. Bull. Chem. Soc. Jpn. 1988, 61, 285. https://doi.org/10.1246/bcsj.61.285

33. Kutschy, P.; Dzurilla, M.; Takasugi, M.; Török, M.; Achbergerová, I.; Homzová, R.; Rácová, M. Tetrahedron 1998, 54, 3549.

https://doi.org/10.1016/S0040-4020(98)00088-X

34. Csomós, P.; Fodor, L.; Sohár, P.; Bernáth, G. Tetrahedron 2005, 61, 9257. https://doi.org/10.1016/j.tet.2005.07.068

35. Jinxian, L.; Shengming, M. Org. Biomol. Chem. 2013, 11, 4186. https://doi.org/10.1039/c3ob40226f

36. Pedras, M. S. C.; Hossain, M.; Sarwar, M. G.; Montaut, S. Bioorg. Med. Chem. Lett. 2004, 14, 5469 .

https://doi.org/10.1016/j.bmcl.2004.09.014

37. Očenášová, L.; Kutschy, P.; Gonda, J.; Pilátová, M.; Gönciová, G.; Mojžiš, J.; Pazdera, P. Chem. Pap. 2016, 70, 635.

38. Mosmann, T. J. Immunol. Methods 1983, 65, 55.

https://doi.org/10.1016/0022-1759(83)90303-4 